Adaptação de viés indutivo de algoritmos de agrupamento de fluxos de dados

Marcelo Keese Albertini 

SERVIÇO DE PÓS-GRADUAÇÃO DO ICMC-USP

Data de Depósito: 7 de maio de 2012

Assinatura:

\title{
Adaptação de viés indutivo de algoritmos de agrupamento de fluxos de dados
}

\author{
Marcelo Keese Albertini
}

Orientador: Prof. Dr. Rodrigo Fernandes de Mello

Tese apresentada ao Instituto de Ciências Matemáticas e de Computação - ICMC-USP, como parte dos requisitos para obtenção do título de Doutor em Ciências - Ciências de Computação e Matemática Computacional. VERSÃO REVISADA 
Ficha catalográfica elaborada pela Biblioteca Prof. Achille Bassi e Seção Técnica de Informática, ICMC/USP, com os dados fornecidos pelo(a) autor(a)

Keese Albertini, Marcelo
K334aptaça de viés indutivo de algoritmos de
agrupamento de fluxos de dados / Marcelo Keese
Albertini; orientador Rodrigo Fernandes de Mello. --
São Carlos, 2012.
134 p.
Tese (Doutorado - Programa de Pós-Graduação em
Ciências de Computação e Matemática Computacional) --
Instituto de Ciências Matemáticas e de Computação,
Universidade de São Paulo, 2012.
1. Aprendizado de Máquina. 2. Agrupamento de
dados. Fluxos de dados. I. Mello, Rodrigo
Fernandes de, orient. II. Título.


À minha esposa, por sua compreensão, carinho, presença e incansável apoio ao longo do período de elaboração deste trabalho. 

À minha família, meu eterno reconhecimento pelos meios que me forneceram para que eu fosse capaz de realizar este trabalho.

Por me guiar com sabedoria e paciência nos sendeiros da pesquisa científica, agradeço imensamente ao meu amigo Rodrigo Fernandes de Mello.

Ao sr. Lucio Medrano Aguirre, meus agradecimentos pelo veemente incentivo à concretização de um ideal acadêmico.

Minha gratidão aos amigos e colegas André Backes, Bruno Feres, Rodrigo Calvo, Cássio Pereira, Pedro Peixoto, Ricardo Rios e Rosane Maffei, que me auxiliaram com valiosas sugestões.

Agradecimentos especiais à Fundação de Amparo à Pesquisa do Estado de São Paulo (FAPESP) pelo contínuo apoio por meio de auxílios científico-financeiros e à Coordenadoria de Aperfeiçoamento de Pessoal de Nível Superior (CAPES) pelo apoio para a realização de um estágio de doutorado no exterior. 



\section{Abstract}

Several research fields have described phenomena that produce endless sequences of samples, referred to as data streams. These phenomena usually present behavior variation and are studied by means of unsupervised induction based on data clustering. In order to cope with the characteristics of data streams, researchers have designed clustering algorithms with low time and space complexity requirements. However, predefined and static parameters (thresholds, number of clusters and learning rates) found in current algorithms still limit the application of clustering to data streams. This limitation motivated this thesis, which proposes a continuous approach to evaluate behavior variations and adapt algorithm inductive bias by changing its parameters. The main contribution of this thesis is the proposal of three approaches to adapt induction bias: i) an approach based on the design of an adaptive artificial self-organizing neural network architecture that enables behavior evaluation by means of Markov chain and Shannon entropy estimations; ii) an approach to adapt traditional data clustering algorithms according to behavior variations in sequences of data chunks; and iii) an approach based on the proposed neural network architecture to continuously adapt parameters by means of the evaluation of data stability. Additionally, in order to analyze the essential characteristics of data streams, this thesis presents a formalization for the problem of data stream clustering and a taxonomy on approaches to detect behavior variations. 



\section{Resumo}

Diversas áreas de pesquisa são dedicadas à compreensão de fenômenos que exigem a coleta ininterrupta de sequências de amostras, denominadas fluxos de dados. Esses fenômenos frequentemente apresentam comportamento variável e são estudados por meio de indução não supervisionada baseada em agrupamento de dados. Atualmente, o processo de agrupamento tem exibido sérias limitações em sua aplicação a fluxos de dados, devido às exigências impostas pelas variações comportamentais e pelo modo de coleta de dados. Embora tem-se desenvolvido algoritmos eficientes para agrupar fluxos de dados, há a necessidade de estudos sobre a influência de variações comportamentais nos parâmetros de algoritmos (e.g., taxas de aprendizado e limiares de proximidade), as quais interferem diretamente na compreensão de fenômenos. Essa lacuna motivou esta tese, cujo objetivo foi a proposta de uma abordagem para a adaptação do viés indutivo de algoritmos de agrupamento de fluxos de dados de acordo com variações comportamentais dos fenômenos em estudo. Para cumprir esse objetivo projetou-se: i) uma abordagem baseada em uma nova arquitetura de rede neural artificial que permite avaliação de comportamento de fenômenos por meio da estimação de cadeias de Markov e entropia de Shannon; ii) uma abordagem para adaptar parâmetros de algoritmos de agrupamento tradicional de acordo com variações comportamentais em blocos sequenciais de dados; e iii) uma abordagem para adaptar parâmetros de agrupamento de acordo com a contínua avaliação da estabilidade de dados. Adicionalmente, apresenta-se nesta tese uma taxonomia de técnicas de detecção de variação comportamental de fenômenos e uma formalização para o problema de agrupamento de fluxos de dados. 

Lista de Figuras vi vi v v v v

Lista de Tabelas $\quad$ vii

Lista de Algoritmos ix

Lista de Símbolos $\quad$ xiii

Glossário $\quad$ Xv

1 Introdução 1

2 Agrupamento de Fluxos de Dados 9

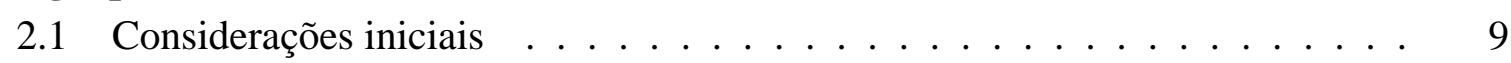

2.2 Histórico da área . . . . . . . . . . . . . . . . . . . 9

2.3 O problema de agrupamento de dados . . . . . . . . . . . . . . . . 12

2.4 O problema de agrupamento de fluxos de dados . . . . . . . . . . . . 15

2.4.1 Análise e projeto de agrupamento para fluxos de dados . . . . . . . . 18

2.5 Algoritmos de agrupamento de fluxos de dados .............. 21

2.5.1 Análise comparativa de algoritmos de agrupamento de fluxos de dados 25

2.6 Considerações finais $\ldots \ldots \ldots \ldots \ldots \ldots$

3 Caracterizacão de Variacão de Comportamento em Fluxos de Dados 29

3.1 Consideracões iniciais . . . . . . . . . . . . . . . . . . . 29

3.2 Conceitos e técnicas . . . . . . . . . . . . . . . . . . 30

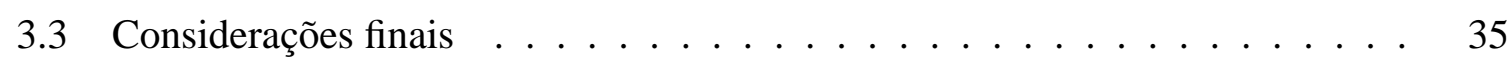

4 Adaptacão de Viés Indutivo de Agrupamento de Fluxos de Dados 37

4.1 Consideracões iniciais . . . . . . . . . . . . . . . . . . 37

4.2 Histórico de desenvolvimento da tese . . . . . . . . . . . . . 38 
4.3 Vieses de algoritmos de agrupamento . . . . . . . . . . . . . . . . 44

4.4 Proposta da arquitetura SondE . . . . . . . . . . . . . . . . . . . . 47

4.5 Caracterizacão de comportamento baseada em Entropia de Shannon e em cadeias de Markov . . . . . . . . . . . . . . . . . . . . . 50

4.6 Primeira abordagem para a adaptacão de viés indutivo . . . . . . . . . . . 53

4.6.1 Análise da convergência de aprendizado . . . . . . . . . . . . 54

4.6.2 Obtencão de um vetor-gradiente da superfície do viés de parâmetros . $\quad 57$

4.6.3 Restricões da adaptacão do viés indutivo . . . . . . . . . . . . . 60

4.6.4 Discussão dos resultados obtidos a partir da primeira abordagem . . . 62

4.7 Segunda abordagem para a adaptacão do viés indutivo . . . . . . . . . . . . . . 62

4.7.1 Descricão da segunda abordagem . . . . . . . . . . . 62

4.7 .2 Algoritmo . . . . . . . . . . . . . . . . . . . . . . . 65

4.7 .3 Comparacão entre algoritmos . . . . . . . . . . . . . . 67

4.7 .4 Experimentos . . . . . . . . . . . . . . . . . . . . . 69

4.7.5 Discussão dos resultados obtidos a partir da segunda abordagem . . . 74

4.8 Terceira abordagem para a adaptacão de viés indutivo . . . . . . . . . . . . . . 74

4.8.1 Descricão da terceira abordagem . . . . . . . . . . . . . 76

4.8 .2 Formulacão . . . . . . . . . . . . . . . . . . . 77

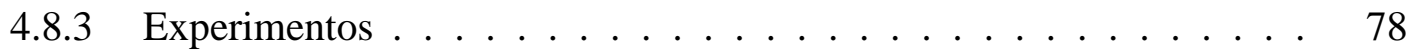

4.8.4 Discussão dos resultados obtidos a partir da terceira abordagem . . . 84

4.9 Considerações finais . . . . . . . . . . . . . . 85

\begin{tabular}{|lll}
5 & Conclusões e trabalhos futuros & 87
\end{tabular}

$\begin{array}{ll}\text { Referências } & 91\end{array}$

\begin{tabular}{|ll}
\hline Apêndice A & 103
\end{tabular}

A.1 Algoritmo da arquitetura Self-Organizing Novelty Detector (SONDE) . . . . . 103

$\begin{array}{ll}\text { Índice Remissivo } & 105\end{array}$ 


\section{Lista de Figuras}

1.1 Descrição do processo indutivo de agrupamento de dados (Xu \& Wunsch, 2005) 2

1.2 Diagrama de componentes da abordagem idealizada no objetivo desta tese . . 6

2.1 Ilustração dos conceitos de agrupamento de dados . . . . . . . . . . . . . . 13

2.2 Ilustração dos conceitos de agrupamento de fluxos de dados . . . . . . . . . . 16

3.1 Árvore de classificação de técnicas proposta por Singh \& Markou (2005) . . . 31

3.2 Árvore hierárquica de detecção de novidades em Aprendizado de Máquina . . 32

1.2 Diagrama de componentes da abordagem idealizada no objetivo desta tese . . 40

4.1 Ilustração de diferentes vieses indutivos de acordo com linguagens de representação de hipóteses $\left(\mathcal{L}_{1}\right.$ e $\left.\mathcal{L}_{2}\right)$, algoritmos indutivos $\left(\mathcal{A}_{1}, \mathcal{A}_{2}, \mathcal{B}_{1}\right.$ e $\left.\mathcal{B}_{2}\right)$ e parâmetros $\left(\phi_{1}\right.$ e $\left.\phi_{2}\right)$. O model ideal $\Gamma_{0}$ e o factível $\Gamma_{1}$ são definidos no conjunto universo de hipóteses U . . . . . . . . . . . . . . . . . . . . . 46

4.2 Arquitetura de rede neural artificial Self-Organizing Novelty Detector (SONDE) 48

4.3 Arquitetura SONDE: ilustração de um neurônio $c_{i}$ e suas estruturas de conhe-

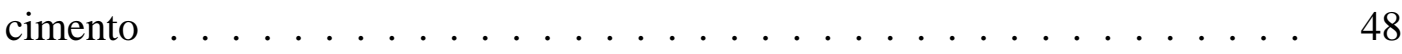

4.4 Experimento de detecção de novidades em uma sequência periódica com três valores . . . . . . . . . . . . . . . . . . . . 52

4.5 Ilustracão do mapeamento de uma partição de agrupamento em estados de uma cadeia de Markov . . . . . . . . . . . . . . . . . . . . . . 63

4.6 Ilustração da abordagem DSDC: coleta e processamento de dados . . . . . . . 66

4.7 Fluxo de dados B: resultados de WCSS e Silhueta . . . . . . . . . . . . . . 72

4.8 Fluxo de dados Control Charts: resultados de WCSS e Silhueta . . . . . . . 73

4.9 Caracterização do número $n$ de padrões necessários para aplicar um fator de esquecimento $\varepsilon \in\{0,0025,0,005,0,01,0,02,0,04\}$ em um padrão passado $\vec{I}(t-n)$, conforme o parâmetro de ponderação $\psi \ldots \ldots$. . . . . . . . 
4.10 Aproximações para uma sequência senoide com ruído Normal $N\left(\mu=0, \sigma^{2}=\right.$ 1). O melhor parâmetro de ponderação inicial $\psi$ para MMIEP, sem a técnica de adaptação atuante, encontrado foi $\psi=0,137$ (distância DTW $=0,089$ ) e o melhor parâmetro de ponderação inicial para a MMEP, com a técnica de adaptação atuante, foi $\psi=0,186$ (distância DTw $=0,121$ ) . . . . . . . . . 79

4.11 Curvas de distância DTw para uma senoide ruidosa de acordo com o parâmetro de ponderação $\gamma$ da arquitetura SONDE, com e sem a técnica de adaptação de viés indutivo atuante . . . . . . . . . . . . . . . . .

4.12 Ilustração de sequências de modelo ideal, padrões de entrada ruidosos, protótipos de neurônios da arquitetura SONDE com e sem a técnica de adaptação atuante. . . . . . . . . . . . . . . . . . 82

4.13 Cenário de aprendizado de senoide estacionária $\ldots$. . . . . . . . . . . . 83

4.14 Cenário de aprendizado de sequência produzida a partir de um modelo estocástico Arima $(1,1,0) \ldots \ldots \ldots$. . . . . . . . . . . . . . . . 84 


\section{Lista de Tabelas}

1.1 Conjuntos tradicionais e fluxos de dados: comparação do processo indutivo .

2.1 Previsões para produção de dados em experimentos científicos (Kramer et al., 2004)

2.2 Avaliação das propriedades de algoritmos de agrupamento de fluxos de dados 26

4.1 Fluxos de dados utilizados na comparação entre DSDC, GwR e K-médias . . 68

4.2 Resultados para DSDC, GWR e K-médias: média e desvio padrão dos valores para o número de grupos $k$, a Silhueta $\mathbf{S}$ e a WCSS . . . . . . . . . . . . 71 



\section{Lista de Algoritmos}

1 Data Streams Dynamic Clustering (DSDC) . . . . . . . . . . . 66

2 Adaptação de parâmetros de uma média móvel exponencial ponderada (MMEP) 78

3 Método de busca pelo valor do parâmetro $\gamma$ que minimiza distância DTw entre os protótipos da arquitetura SONDE para um fluxo de dados e o modelo ideal . $\quad 80$

4 Método para obter a distância DTw para uma instância da SONDE . . . . . . . 80

5 Algoritmo para a implementação da arquitetura de rede neural artificial Self-Organizing Novelty Detector . . . . . . . . . . . . . . . . . . . . . . . . . . . 104 



\section{Lista de Símbolos}

$\alpha_{i}$

$\delta$

$\eta$

$\Gamma$

$\gamma$

$\gamma_{i}, \Omega_{i}$

$\mathbb{H}(t)$

L

M

$\mathrm{U}$

$\mathcal{A}, \mathcal{B}, \mathcal{L}$

$\nabla f(x, y)$

$\Omega$

Sequência de partições $\{\Gamma(-\infty), \ldots, \Gamma(0)\}$

Nível de ativação para um padrão de entrada, referente ao $i$-ésimo neurônio da arquitetura SONDE

Erro de estimação

Taxa de aprendizado

Partição de um conjunto de elementos $E=\left\{\left\{e_{0}, \ldots\right\},\left\{e_{1}, e_{2}, \ldots\right\}, \ldots\right\}$ em subconjuntos disjuntos, ou seja, um agrupamento

Parâmetro de ponderação de aprendizado de protótipo da arquitetura SONDE

Parâmetros de aprendizado da arquitetura SONDE associados ao neurônio $c_{i}$

Entropia de Shannon de uma matriz de probabilidades de transição de uma cadeia de Markov estimada no instante de tempo $t$

Matriz de contagem de ativações consecutivas entre neurônios

Matriz de probabilidades de transição entre estados de uma cadeia de Markov

Conjunto universo de partições de um conjunto

Subespaços de hipóteses ou conjuntos de partições

Vetor-gradiente de uma função

Parâmetro de ponderação de aprendizado de raio médio da arquitetura SONDE 
Subespaço de hipóteses definido pelo viés indutivo de parâmetros de um algoritmo de agrupamento

Função de distribuição de probabilidades

Parâmetro de ponderação de uma MMEP

Dispersão média de padrões de entrada em relação ao protótipo de seu grupo $c_{i}$

Função de proximidade de vizinhança ao neurônio BMU em uma rede neural artificial auto-organizável

Relação alcançar entre duas partições $\Gamma \triangleright \Upsilon$

Fator de esquecimento aplicado a um padrão de entrada em uma MMEP

Coeficiente de esquecimento da neural neural artificial RSOM

Vetor ou padrão de entrada obtido no instante de tempo $t$

Média móvel exponencial ponderada no instante de tempo $t$

Protótipo vetorial referente ao $i$-ésimo agrupamento de dados

Protótipo do BMU obtido no instante de tempo $t$

Norma euclidiana de um vetor de números reais

Nível de confiança para uma estimativa

Cadeia de Markov $C=(V, T)$ com um conjunto $V$ contendo $k$ estados comportamentais e um conjunto $T$ de transições entre estados

$i$-ésimo agrupamento de dados

Matriz de distâncias ou dissimilaridades

Matriz de distâncias proveniente de uma transformação de outra matriz de distâncias $d$

Conjunto finito e não ordenado de dados $E=\left\{e_{0}, e_{1}, e_{2}, \ldots\right\}$

Função-objetivo para a minimização da variação de entropia e adaptação do viés indutivo da arquitetura SONDE

Função de agrupamento para um fluxos de dados $S(t)$, utilizando uma matriz ou função de distâncias $d$

Função de agrupamento $f$, que mapeia uma matriz de distâncias $d$ entre elementos de um conjunto a uma partição $\Gamma$ pertencente ao conjunto universo de partições $\mathbf{U}$ 


$\begin{array}{ll}I(x, y) & \text { Medida de representatividade de um protótipo y para um grupo } x \\ k & \text { Número de grupos } \\ l & \text { Número de atributos de um vetor ou padrão de entrada } \\ N\left(\mu, \sigma^{2}\right) & \text { Função de distribuição de probabilidades Normal com média } \mu \text { e vari- } \\ & \text { ância } \sigma^{2} \\ p_{i, j} & \text { Probabilidade de transição entre um estado } i \text { para um estado } j \text { de uma } \\ & \text { cadeia de Markov } \\ p_{i} & \text { Probabilidade de um sistema representado por uma cadeia de Markov } \\ & \text { assintoticamente convergir ao estado } i \\ S & \text { Fluxo de dados } S=\{s(-\infty), \ldots, s(t), \ldots, s(\infty)\}, \text { um conjunto infi- } \\ & \text { nito e ordenado de dados de acordo com o instante de tempo ou ordem } \\ & \text { de coleta } t \\ & \text { Elemento de uma sequência referente ao instante de tempo ou ordem } \\ & \text { de coleta } t\end{array}$



A RIMA Autoregressive Integrated Moving Average

ART Adaptive Resonance Theory

BMU Best-Matching Unit

DLBLC Diffuse Large B-cell Lymphoma

DSDC Data Stream Dynamic Clustering

DTw Dynamic Time Warping

GWR Grow When Required

Нмм Hidden Markov Models

KNN K-Nearest Neighbors

MLP Multi-Layer Perceptron

MMEP Média móvel exponencial ponderada

RBF Radial Basis Function Network

RSOM Recurrent Self-Organizing Maps

SMART Self-Monitoring, Analysis and Reporting Technology

SoM Self-Organizing Maps

SONDE Self-Organizing Novelty Detector

SvM Support-Vector Machine

WCSS Within-Cluster Sum of Squares 


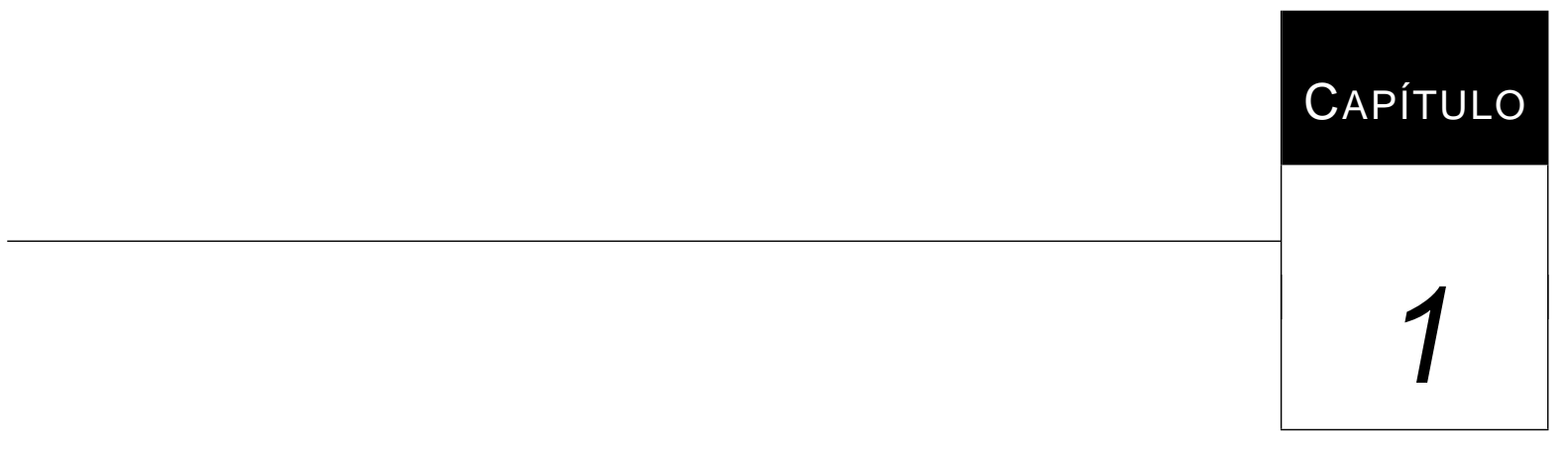

\section{Introdução}

De acordo com o filósofo Kant (1781), a compreensão racional de fenômenos que nos circundam, tais como as interações entre corpos celestes (Newton, 1687; Tyson et al., 2002), a trajetória de objetos (Bush, 1931), o clima e o tempo (Moura, 1996), depende completamente da capacidade humana de coleta e análise de dados. Antes do advento dos computadores, a compreensão de fenômenos era limitada à habilidade pessoal de, manualmente, analisar dados com base em conhecimentos sobre o mundo. Desde o início do desenvolvimento dos primeiros computadores, buscou-se automatizar a execução de tarefas repetitivas para auxiliar no processamento de dados (Bush, 1931). Com o uso dessas ferramentas, foi possível o projeto de métodos de análise, anteriormente inviáveis, que dependem da execução de grandes quantidades de operações lógicas e aritméticas. Dessa forma, a transferência da responsabilidade de executar cálculos a essas máquinas permitiu a concentração de esforços no projeto de avançadas técnicas de compreensão de fenômenos. Essa evolução na maneira de obter conhecimento possibilitou o estudo de fenômenos mais complexos, a partir da coleta de quantidades de dados cada vez maiores (Hey et al., 2009).

Atualmente, existe um processo bem estabelecido para a compreensão de fenômenos. Esse processo é denominado indução e tem por objetivo, a partir da coleta de dados, generalizar tendências e criar modelos que descrevam propriedades do fenômeno observado. Processos de indução podem ser baseados em métodos supervisionados e não supervisionados (Mitchell, 
1997). Métodos supervisionados são úteis em atividades de reconhecimento e classificação de padrões previamente estabelecidos, enquanto que métodos não supervisionados são adequados para o estudo de fenômenos pouco conhecidos.

Para a compreensão de fenômenos pouco conhecidos, em que não há informações prévias obtidas por especialistas, o método não supervisionado mais utilizado é o Agrupamento de Dados (Xu \& Wunsch, 2005). O objetivo desse método é encontrar e distinguir propriedades relevantes a partir da avaliação da similaridade, ou dissimilaridade, entre dados (Jain et al., 1999; Jain, 2010). Um agrupamento ${ }^{1}$ é, essencialmente, uma organização de um conjunto de elementos $E=\left\{e_{0}, e_{1}, e_{2}, \ldots\right\}$ na forma de uma partição $\Gamma$ (Gama) composta de subconjuntos representativos $\Gamma=\left\{\left\{e_{0}, \ldots\right\},\left\{e_{1}, e_{2}, \ldots\right\}, \ldots\right\}$. Cada elemento $e \in E$ é constituído a partir de dados que são organizados na forma de atributos relevantes e utilizados na comparação da dissimilaridade entre elementos. O processo indutivo baseado em agrupamento de dados, ilustrado na Figura 1.1, é usualmente organizado nas seguintes etapas (Xu \& Wunsch, 2005): primeiramente, a coleta de dados a partir da observação do fenômeno em estudo; a seguir, a seleção ou extração de atributos relevantes dos dados; em terceiro lugar, o projeto ou escolha de um algoritmo de agrupamento; após isso, a execução do algoritmo e as etapas de validação do modelo obtido, ou seja, o agrupamento, e interpretação dos resultados; e, por fim, a obtenção de conhecimento sobre o fenômeno observado.

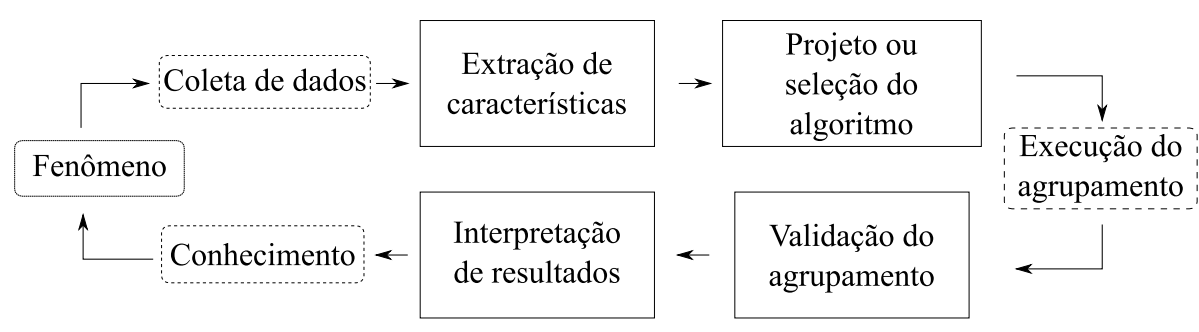

Figura 1.1: Descrição do processo indutivo de agrupamento de dados (Xu \& Wunsch, 2005)

O planejamento e a execução de um processo de agrupamento é uma atividade complexa que envolve várias decisões estratégicas. As principais são realizadas no projeto do algoritmo indutivo, ou indutor, e da linguagem de representacão de hipóteses (Dehaspe \& Raedt, 1996), que, no caso de agrupamento de dados, define as relações entre elementos e determina a formação de grupos. Essas decisões são essenciais para a obtenção de modelos, ou seja, agrupamentos descritos por conjuntos de hipóteses adequadas para representar o fenômeno de interesse. Cada combinação de indutor e linguagem de representação de hipóteses deve considerar as necessidades provenientes das características do fenômeno observado. Essas necessidades são consideradas para a determinação de premissas e suposições a fim de orientar o processo indutivo, as quais delimitam um subespaço do conjunto universo de hipóteses em que o indutor buscará por modelos. Por exemplo, para elementos observados

\footnotetext{
${ }^{1}$ Em agrupamentos nebulosos (em inglês, fuzzy), um elemento pode pertencer a vários grupos simultaneamente.
} 
em um espaço métrico, pode-se utilizar um algoritmo de agrupamento, ou seja, um indutor, que visa minimizar a soma de distâncias quadráticas e uma linguagem de representação de hipóteses baseada em uma norma euclidiana para a definição de proximidades. Premissas e suposições como essas constituem o viés indutivo do algoritmo (Mitchell, 1997) e são elementos-chave para o sucesso do processo de agrupamento e para a obtenção de uma boa representação dos dados.

Atualmente, o processo indutivo de agrupamento considerado tem exibido sérias limitações em sua aplicação a diversos fenômenos que exigem a rápida coleta e processamento de grandes volumes de dados (Pavlidis et al., 2011). Para muitos desses fenômenos, dados são apropriadamente organizados na forma de sequências ilimitadas denominadas fluxos de dados (O’Callaghan et al., 2002). Um fluxo de dados (Sousa \& Traina, 2006; Sun \& Lu, 2006) é representado por uma sequência $S=\{s(-\infty), \ldots, s(t), \ldots, s(\infty)\}$ de elementos $s(t)$ coletados pela observação contínua de fenômenos em contextos computacionalmente intensivos, tais como em análises climatológicas (Allcock et al. . 2001: Sant'anna Neto, 2003; Korting et al. 2009); em mineracão de documentos publicados na Internet (Bovnton, 2010; Masud et al., 2010; Lindstrom et al., 2010); e em experimentos científicos avancados (Swenson Jr \& Kellermann, 1975; Tyson et al., 2002).

Existem dois tipos de fenômenos apropriadamente representados por fluxos de dados (Guha et al., 2003). O primeiro tipo é caracterizado pela grande quantidade de dados acumulada e pela exigência de rápida obtenção de modelos. Nesse caso, dados são armazenados em memórias secundárias, caracterizadas por baixa taxa de transferência, cujo acesso deve ser linear. O segundo tipo é mais exigente em termos computacionais. Nele, os dados são coletados a altas taxas e quantidades, o que impossibilita seu armazenamento e exige seu descarte após processamento. A coleta e descarte de dados implica na existência de uma relação de ordem entre esses elementos. Para esse tipo de fenômeno, agrupamentos devem ser continuamente induzidos ao longo do processo de coleta.

As principais diferenças entre o processo de agrupamento aplicado a conjuntos de dados finitos e não ordenados, i.e., conjuntos tradicionais de dados, e o agrupamento de fluxos de dados estão resumidas na Tabela 1.1 (O'Callaghan et al., 2002). Em primeiro lugar, o tamanho e o tipo de acesso a fluxos de dados diferem dos conjuntos tradicionais, que são completamente armazenáveis em memória e permitem acesso aleatório. Em segundo lugar, a duração indeterminada de fluxos reflete na continuidade e necessidade de adaptação do processo indutivo. Outra diferença refere-se à natureza do modelo induzido. Enquanto, no processo tradicional, o agrupamento é raramente modificado após a conclusão do processo, em fluxos de dados, a natureza do agrupamento é transiente devido à coleta e processamento contínuos. Finalmente, a participação de especialistas do fenômeno de interesse no processo indutivo de fluxos de dados é diferente da participação no processo tradicional. No tradicional, especialistas são necessários para a seleção e extração de características relevantes dos dados; 
para a escolha da linguagem de representação de hipóteses do algoritmo indutivo e para a validação do agrupamento obtido. Por outro lado, em fluxos de dados, especialistas estão presentes principalmente no projeto do processo indutivo e, pela natureza infinita, tipicamente, não são capazes de acompanhá-lo.

Tabela 1.1: Conjuntos tradicionais e fluxos de dados: comparação do processo indutivo

\begin{tabular}{ccc}
\hline Característica & Conjuntos tradicionais & Fluxos de dados \\
\hline Tamanho & Limitado & Ilimitado, excede capacidade de memória \\
Acesso & Irrestrito & Limitado, como uma sequência \\
Modo de aprendizado & Estático & Contínuo \\
Natureza do modelo & Estável & Transiente \\
Especialistas & Presentes & Presentes apenas no projeto \\
\hline
\end{tabular}

Pesquisas sobre o método de agrupamento aplicado a fluxos de dados têm se concentrado em prover algoritmos cuja execução apresenta baixa complexidade de tempo e espaço para atender à alta taxa e grande volume de coleta de dados (Xu \& Wunsch, 2005). A maior parte dos algoritmos atuais busca lidar com o primeiro tipo de fluxo de dados, i.e., grandes conjuntos de dados armazenados em memória secundária, com o objetivo de atender suas restrições computacionais e, em geral, desconsideram a natureza sequencial desses elementos (Sheikholeslami et al., 1998). Essas pesquisas assumem que, caso o processo indutivo seja inicialmente bem projetado, agrupamentos serão incrementalmente construídos e a qualidade de suas hipóteses aumentarão em função da coleta de mais dados, ou seja, mais evidências que confirmam, refinam ou refutam as hipóteses. Essa premissa é verdadeira quando as características internas, ou seja, o comportamento do fenômeno observado, são estáveis, o que ocorre com frequência para conjuntos tradicionais de dados.

A premissa de estabilidade do fenômeno não é adequada para a maioria dos fluxos de dados do segundo tipo, que exigem o processamento de dados ao longo do processo de coleta. Variações de comportamento do fenômeno provocam modificações nas características dos dados. Tais modificações, conhecidas como concept drift (Hulten et al., 2001), influenciam na validade de decisões e premissas do viés indutivo (Kifer et al., 2004; Pavlidis et al., 2011) e acentuam as dificuldades envolvidas no projeto de algoritmos de agrupamento, pois, uma vez que o viés indutivo esteja comprometido, a qualidade do agrupamento pode deteriorar-se.

As dificuldades apresentadas pela presença de variações comportamentais em fluxos de dados têm sido abordadas pelo desenvolvimento de técnicas adaptativas na área de Aprendizado de Máquina, tais como algoritmos baseados no paradigma Winner Takes All (Fritzke, 1995; Biehl et al., 2006; Young et al., 2010) e arquiteturas de redes neurais artificiais baseadas em mapas auto-organizáveis, e.g., a rede Grow When Required (GWR) (Marsland, 2002) e a arquitetura de rede neural artificial Adaptive Resonance Theory (Carpenter et al., 1991). Algoritmos de agrupamento para fluxos de dados que implementam o paradigma Winner Takes All incrementalmente adaptam grupos de acordo com heurísticas de aprendizado, enquanto 
mapas auto-organizáveis empregam modelos inspirados em fenômenos biológicos, tal como a habituação (Stanley, 1976), utilizado pela rede GWR para dinamicamente criar novos neurônios e representar variações comportamentais.

Na rede GWR, assim como em outras abordagens atuais, parâmetros ${ }^{2}$ são empiricamente definidos por especialistas de acordo com conhecimento prévio ou por meio da utilização de meta-heurísticas e mantidos constantes durante o processo de agrupamento. Dessa forma, quando ocorrem variações de comportamento, é possível que as novas características do fenômeno observado violem as suposições e premissas estabelecidas no viés indutivo durante a etapa de projeto. Dependendo das novas características do fenômeno, a adaptação de grupos torna-se ineficaz em manter uma representação adequada para o novo comportamento observado.

Apesar da importância de produzir agrupamentos representativos ao longo do tempo, atualmente se conhece pouco sobre a associação entre variações de comportamento de fenômenos e a adaptação automática do viés indutivo de algoritmos de agrupamento de fluxos de dados (Brazdil, 2009).

A necessidade de compreender fenômenos observados a partir de fluxos de dados com variações comportamentais e a falta de técnicas que continuamente adaptam parâmetros de algoritmos de agrupamento motivaram a definição da hipótese desta tese de doutorado:

Hipótese: Adaptando o viés indutivo de algoritmos de agrupamento de fluxos de dados de acordo com variações comportamentais do fenômeno em estudo pode-se garantir a contínua obtenção de conhecimento.

Essa hipótese motivou estabelecer como objetivo desta tese:

Objetivo: Projetar e desenvolver uma abordagem de adaptação do viés indutivo de parâmetros de algoritmos de agrupamento de fluxos de dados, considerando fenômenos que apresentam variação comportamental.

A abordagem idealizada nesse objetivo foi estabelecida, conforme a Figura 1.2, para conter três principais componentes: i) um algoritmo de agrupamento de fluxos de dados; ii) uma medida para avaliar variações comportamentais presentes em fluxos de dados; iii) e uma

\footnotetext{
${ }^{2}$ Parâmetros também são conhecidos como hiperparâmetros, conforme utilizado no campo de pesquisa de Meta-aprendizado para distingui-los de parâmetros estatísticos (Mitchell, 1997).
} 
técnica de adaptação de viés indutivo por meio da modificação de parâmetros do algoritmo de agrupamento, de acordo com variações comportamentais do fenômeno observado.

(i)

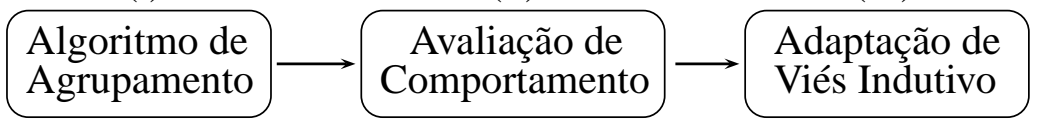

Figura 1.2: Diagrama de componentes da abordagem idealizada no objetivo desta tese

Ao longo do desenvolvimento desta tese, encontraram-se diferentes opções de projeto para o objetivo idealizado, dentre as quais destacam-se três abordagens que auxiliaram na comprovação da hipótese previamente estabelecida.

Os estudos realizados para a primeira abordagem basearam-se, conforme o diagrama apresentado na Figura 1.2, em: i) projeto de uma arquitetura de rede neural artificial autoorganizável denominada Self-Organizing Novelty Detector (SONDE) para agrupar dados de maneira adaptativa e incremental; ii) medição de variações comportamentais por meio da estimação de cadeias de Markov (Seneta, 2006) e da entropia de Shannon (1948); e iii) projeto de uma técnica de adaptação de viés indutivo por meio da minimização da variação de entropia de cadeias de Markov ocorrida devido à inadequação dos parâmetros que constituem o viés da arquitetura SONDE

Nessa primeira abordagem, descrita na Seção 4.6, a arquitetura projetada SONDE permitiu representar grupos por meio de equações de aprendizado baseadas em proximidade espacial entre elementos, as quais possuem parâmetros que constituem seu viés indutivo. A representação de grupos por meio de neurônios da SONDE permitiu a estimação de probabilidades de transições de cadeias de Markov, que é a base para o cálculo de uma medida de caracterização comportamental de fenômenos baseada em variação da entropia de Shannon (1948). Essa medida foi utilizada no projeto de uma técnica de adaptação de viés indutivo inspirada em um método de otimização estocástico de gradiente descendente. Nessa técnica, a função gradiente da variação de entropia em relação aos parâmetros da SONDE indicaria a intensidade em que tais parâmetros seriam adaptados. Porém, apesar da concentração de esforços para o desenvolvimento e refinamento dessa abordagem, ela não foi bem sucedida. Isso ocorreu principalmente devido a limitações encontradas na obtenção da relação entre variações de entropia e os parâmetros da SONDE, necessária à definição da função de gradiente utilizada no método de otimização.

Posteriormente, buscou-se outras alternativas para definir a relação entre o algoritmo de agrupamento e a adaptação do viés indutivo. Essa busca resultou em uma segunda abordagem que constituiu, conforme o diagrama na Figura 1.2, da: i) utilização do algoritmo de agrupamento para conjuntos tradicionais de dados K-médias (Hartigan, 1975) para a produção de partições em blocos de subsequências; ii) estimação de propriedades assintóticas de cadeias 
de Markov para a verificação de equivalência de comportamento entre partições consecutivas (Ornstein, 1989; Seneta, 2006); e iii) aplicação de um algoritmo de otimização que define o número de grupos para o algoritmo K-médias de acordo com a medida de qualidade de partições Silhueta (Rousseeuw, 1987).

Essa segunda abordagem, denominada Data Stream Dynamic Clustering, foi bem sucedida em adaptar o viés indutivo de acordo com o reconhecimento da variação de comportamento entre blocos de dados (Albertini \& Mello, 2013). Contudo, conforme mencionado na hipótese desta tese, para manter a efetiva obtenção de conhecimento sobre fenômenos é necessária a contínua avaliação dos dados. Dessa forma, o uso de algoritmos de agrupamento baseados em blocos de dados, que não distinguem a ordem de coleta de dados pertencentes a um mesmo bloco, prejudica a compreensão de variações de comportamento de fenômenos.

A adaptação contínua do viés indutivo de algoritmos para efetivamente manter atualizado o conhecimento sobre o comportamento do fenômeno em estudo motivou estudos para a proposta de uma terceira abordagem definida no objetivo desta tese. Esses estudos basearam-se, conforme a Figura 1.2, em: i) utilização da arquitetura SONDE como algoritmo de agrupamento; ii) emprego de uma medida de estabilidade de comportamento presente no fluxo de dados; e iii) projeto de uma técnica de adaptação de viés indutivo baseada na relação entre a medida de estabilidade e os parâmetros das equações de aprendizado de estruturas de conhecimento da arquitetura SONDE,

A proposta da terceira abordagem partiu da associação dos parâmetros das equações de aprendizado da SONDE à necessidade de representar a dependência temporal entre dados, de acordo com a estabilidade de comportamento observada nos dados. Para isso, projetou-se uma técnica de adaptação baseada em uma função de mapeamento entre a medida de estabilidade de dados e os parâmetros das equações da arquitetura SONDE. Essa técnica atua sobre os parâmetros de forma a aumentar a memória das equações de aprendizado, caso dados apresentem maior estabilidade e diminuir, caso contrário. Resultados obtidos a partir de experimentos realizados com fluxos de dados sintéticos, utilizando essa técnica, permitiram verificar as vantagens da adaptação contínua do viés indutivo de agrupamento, comprovando a hipótese desta tese.

Adicionalmente, ao longo do projeto das três abordagens previamente descritas, foi observada a necessidade de compreender o problema de agrupamento de fluxos de dados de maneira mais aprofundada. Para isso, realizaram-se estudos baseados em uma formalização do problema de agrupamento de conjuntos tradicionais de dados (Kleinberg, 2002) para estabelecer, nesta tese, uma formalização similar para agrupamento de fluxos de dados. Essa formalização auxiliou na compreensão das propriedades únicas ao agrupamento de fluxos e na caracterização da natureza do viés indutivo de algoritmos de agrupamento. Contudo, ainda se verifica a possibilidade de realização de trabalhos futuros para ampliar a formalização de propriedades de algoritmos e estender a adaptação do viés indutivo de algoritmos de agrupa- 
mento de fluxos de dados. A execução desses trabalhos futuros está prevista para um estágio de pós-doutorado a ser realizado no Instituto de Ciências Matemáticas e de Computação da Universidade de São Paulo, com o apoio da Fundação de Amparo à Pesquisa do Estado de São Paulo, processo n. 2011/19459-8, sob supervisão do prof. Rodrigo Fernandes de Mello.

A seguir, esta tese de doutorado apresenta o estado da arte em agrupamento de fluxos de dados, estudos sobre a caracterização de variações de comportamento de fenômenos e as atividades desenvolvidas para a definição de uma abordagem de adaptação de viés indutivo para agrupamento de fluxos de dados. De acordo com esses itens, esta tese está organizada nos seguintes capítulos:

Capítulo 2-Agrupamento de Fluxos de Dados: esse capítulo apresenta o estado da arte sobre agrupamento em fluxos de dados, incluindo um estudo sobre os algoritmos mais relevantes;

Capítulo 3 - Caracterização de Variação de Comportamento em Fluxos de Dados: esse capítulo descreve conceitos e trabalhos em diferentes áreas do conhecimento para a caracterização de variações de comportamento de fenômenos representados por meio de fluxos de dados;

Capítulo 4-Adaptação de Viés Indutivo de Algoritmos de Agrupamento de Fluxos de Dados: esse capítulo é dedicado aos estudos realizados para o projeto e desenvolvimento de abordagens para a adaptação de viés indutivo de parâmetros de algoritmos de agrupamento de fluxos de dados; e

Capítulo 5-Conclusões e trabalhos futuros. 


\section{2}

\section{Agrupamento de Fluxos de Dados}

\subsection{Considerações iniciais}

Este capítulo introduz o método de agrupamento de dados e conceitos relacionados. Devido às características que distinguem fluxos de dados de conjuntos tradicionais de dados, propõe-se uma formalização do problema de agrupamento de fluxos de dados com base em conceitos da Teoria dos Conjuntos (Royden, 1968). Essa formalização provê definições para a análise teórica de algoritmos de agrupamento de fluxos de dados de maneira generalizada e objetiva, conforme estabelecido por propriedades básicas para o seu projeto. Posteriormente, a atual literatura de agrupamento de fluxos de dados é apresentada.

\subsection{Histórico da área}

O projeto dos primeiros métodos automáticos para agrupamento de dados ocorreu na década de 1950 (Jain, 2010). Nessa época, buscava-se a resolução de problemas de natureza exploratória com conjuntos de dados pouco conhecidos, o que envolvia, entre outras atividades, a detecção de anomalias, a identificação de características predominantes em fenômenos, a obtenção de classificações naturais de organismos e a representação de dados por meio de 
sua organização (Ward, 1963 ).

Esses problemas surgiram a partir de questionamentos de natureza prática, tais como: a seleção de pessoas de acordo com características socioeconômicas; identificação de históricos profissionais similares para minimizar o tempo de treinamento de trabalhadores realocados; e a organização de conjuntos de modelos matemáticos com o objetivo de reduzir suas imprecisões (Ward, 1963).

Nessa época, verificou-se que era possível abordar manualmente ou visualmente tais problemas apenas quando o número de elementos era pequeno, tipicamente abaixo de cem, e descritos por meio de, no máximo, dois atributos (também conhecidos como características, dimensões ou, em inglês, features). Porém, problemas práticos frequentemente apresentam mais de dois atributos a serem avaliados e um maior número de elementos, o que motivou a criação de técnicas computacionais que automatizassem a execução dessas tarefas (Ward, 1963).

O problema de agrupamento de dados foi abordado por diversas áreas do conhecimento, sob diferentes pontos de vista, conceitos e metodologias, sendo também denominado $Q$-analvsis em Ciências Sociais, tipologia em Psicologia e taxonomia em Biologia (Jain \& Dubes, 1988).

Apesar da diversidade de metodologias e conceitos utilizados, algoritmos de agrupamento podem ser, de modo geral ${ }^{1}$, divididas em dois tipos (Jain, 2010; Xu \& Wunsch, 2005): hierárquicas e partitivas.

Técnicas hierárquicas têm como objetivo construir hierarquias de agrupamento a partir da análise sucessiva de grupos. Em geral, essas técnicas podem utilizar duas abordagens para a construção de hierarquias. A abordagem denominada divisiva inicialmente assume que todos os dados pertencem a apenas um grupo e, em seguida, sucessivamente divide dados em grupos os mais distintos possíveis. Por outro lado, a segunda abordagem, conhecida como aglomerativa, considera que cada elemento constitui seu próprio grupo e os une, conforme a similaridade de seus elementos, até que reste apenas um grupo contendo todos os elementos. Nessas abordagens, uma medida de avaliação para unir ou separar grupos deve ser definida, e.g., a distância euclidiana entre os elementos mais próximos de diferentes grupos. Uma hierarquia de agrupamentos obtidos a partir desses algoritmos é denominada dendrograma. No final da execução do agrupamento, deve-se estabelecer um critério de corte do dendrograma para obter a partição desejada, tais como, um número mínimo de elementos por grupo ou uma distância máxima entre elementos do mesmo grupo. A maior parte de algoritmos de agrupamento hierárquico são baseadas na proposta de Ward (1963) e, frequentemente, buscam reduzir custos computacionais relacionados à complexidade de tempo e espaço, redefinir critérios de

\footnotetext{
${ }^{1}$ Existem outras formas de comparar algoritmos de agrupamento. Xu \& Wunsch (2005) reclassificam os métodos partitivos em sete subtipos. Ilango \& V. (2010) diferenciam agrupamentos em métodos baseados em densidade, grade e modelos.
} 
corte de dendrograma e funções de distância.

Técnicas partitivas organizam dados de acordo com o número desejado de grupos. Tal abordagem originou-se com o algoritmo K-médias (Steinhaus, 1956), cujo objetivo é separar um conjunto de dados $E$ em $k$ subconjuntos $c_{i}(i=1,2, \ldots, k)$, i.e., grupos, de modo que a escolha de um elemento representativo $w_{i}$, denominado protótipo, para cada grupo $c_{i}$, maximize a soma $\sum_{i=1}^{k} I\left(c_{i}, w_{i}\right)$, em que $I(x, y)$ designa, de maneira geral, uma função que mensura a representatividade do protótipo $w_{i}$ para seu grupo $c_{i}$. Outras técnicas partitivas apresentam formulações distintas, como, por exemplo, a identificação da mistura de funções de distribuição de probabilidades e a busca por subconjuntos de elementos definidos de acordo com a densidade de sua vizinhança (Xu \& Wunsch, 2005).

Com o passar do tempo, a evolução das tecnologias de coleta de dados exigiu o aperfeiçoamento e a especialização de algoritmos de agrupamento de dados (Jain, 2010). Aplicações com grande número de atributos, como em análise genômica (Lukashin et al., 2003), motivaram o desenvolvimento de algoritmos que selecionam e agrupam apenas parte desses atributos. Para abordar problemas em que a concentração espacial é mais importante que a distância entre exemplos, tal como em segmentação de imagens, desenvolveram-se métodos baseados em densidade (Ester et al., 1996). Além disso, para a comparação de dados de natureza não estruturada, tais como áudio, imagens e textos (Banerjee et al., 2005), uma grande variedade de técnicas foi criada.

A partir da metade da década de 1990, a contínua evolução da capacidade de coleta de dados com o objetivo de compreensão de fenômenos complexos exerceu uma grande pressão no desenvolvimento de métodos computacionais eficientes (Hey et al., 2009; Kramer et al., 2004; Dewdney et al., 2009). Essa tendência é ilustrada na Tabela 2.1, a qual apresenta projeções realizadas em meados da década de 2000 que prevêm um aumento da produção de dados em até 125 vezes em um período de 5 anos (Kramer et al., 2004).

Tabela 2.1: Previsões para produção de dados em experimentos científicos Kramer et al. 2004)

\begin{tabular}{cccc}
\hline Disciplina científica & Até 5 anos & Mais que 5 anos & Mais que 10 anos \\
\hline Simulações climáticas & até $40 \mathrm{~TB}$ & 1TB por ano, até $5 \mathrm{~PB}$ & ordem de $10 \mathrm{~PB}$ \\
Física de alta energia & até $1.2 \mathrm{~TB}$ por ano & 1PB por ano & ordem de $1000 \mathrm{~PB}$ \\
Fusão magnética & até $1 \mathrm{~TB}$ por ano & 100TB por ano & centenas de TB \\
Química & até $30 \mathrm{~TB}$ & até $100 \mathrm{~TB}$ & até $1 \mathrm{~PB}$ \\
Bioinformática & ordem de $1 \mathrm{~TB}$ & - & ordem de $1 \mathrm{~PB}$ \\
\hline
\end{tabular}

Fonte: Kramer et al. (2004). Deep scientific computing requires deep data. IBM Journal of Research and Development, v.48, n.2, p.209-232.

Atualmente, algoritmos de agrupamento têm demonstrado sérias limitações quando aplicados para a compreensão de diversos fenômenos que exigem a rápida coleta e processamento de grandes volumes de dados (Pavlidis et al., 2011; Guha et al., 2003). Para muitos desses fenômenos, dados são apropriadamente organizados na forma de sequências ilimitadas 
denominadas fluxos de dados (O’Callaghan et al., 2002). Fluxos de dados são sequências de observações $s(t)$ ordenadas na forma $S=\{s(-\infty), \ldots, s(t), \ldots, s(\infty)\}$ obtidas em contextos computacionalmente intensivos, tais como em análises climatológicas e geográficas (Allcock et al., 2001; Heas \& Datcu, 2005; Boriah et al., 2008; Mota et al., 2009); em extração de informação de documentos publicados na Internet (Masud et al., 2010; Lindstrom et al., 2010); e em experimentos científicos avançados (Swenson Jr \& Kellermann, 1975; Tyson et al., 2002).

Existem dois tipos de fenômenos apropriadamente representados por fluxos de dados (Guha et al., 2003). O primeiro tipo é caracterizado pelo grande volume de dados e pela exigência de rápida obtenção de modelos. Nesse caso, dados são armazenados em memória secundária, com baixa taxa de transferência, cujo acesso deve ser linear (não aleatório) e restrito a um número baixo de operações. Exemplos desse primeiro tipo incluem bases de dados médicas (Daura et al., 1998) e socioeconômicas (Aggarwal et al., 2003).

O segundo tipo é ainda mais exigente em termos computacionais. Nele, dados são coletados a altas taxas e quantidades, o que impossibilita seu armazenamento e exige seu descarte após processamento. Para esse tipo de fenômeno, modelos induzidos devem ser adaptados ao longo do processo de coleta. Nesse segundo tipo de fluxo, incluem-se aplicações de detecção de intrusão em redes de computadores (Aggarwal et al., 2003), análise de tendências de microblogging (Conover et al., 2011; Mustafaraj et al., 2012) e registros de chamadas telefônicas (Cortes \& Pregibon, 2001).

Essas características de fluxos de dados impõem condições especiais ao desenvolvimento de algoritmos de agrupamento de conjuntos finitos e não ordenados de dados, i.e., conjuntos tradicionais de dados (Cortes \& Pregibon, 2001), conforme apresentado na Tabela 1.1. Nota-se, portanto, que os aspectos relevantes para o problema de agrupamento para esses dois casos são distintos. Em respeito à definição do problema computacional envolvido no caso de conjuntos tradicionais, a Seção 2.3 apresenta uma formalização proposta por Kleinberg (2002). A seguir, propõe-se, na Seção 2.4, uma formalização do problema de agrupamento para fluxos de dados que estende as propriedades básicas para algoritmos de agrupamento propostas por Kleinberg (2002). Essa formalização permite que, conforme apresentado na Seção 2.5, algoritmos de agrupamento de fluxos de dados sejam avaliados e comparados em termos das propriedades propostas nesta tese.

\subsection{O problema de agrupamento de dados}

Kleinberg (2002) considerou elementos da Teoria dos Conjuntos (Royden, 1968) para descrever algoritmos de agrupamento independentemente de conceitos específicos de aplicações ou técnicas utilizadas. Essa análise é importante para compreender algoritmos de antemão, i.e., sem a necessidade de ensaios experimentais, e para fornecer diretrizes de projeto 
para novas abordagens.

Os conceitos envolvidos na formalização de Kleinberg são ilustrados na Figura 2.1 . Essa formalização assume que elementos são organizados em um conjunto $E$, os quais podem ser comparados por meio de uma matriz de dissimilaridades ou distâncias. Tal matriz $d$ é descrita na forma de uma função $d: E \times E \rightarrow \mathbb{R}^{+}$e é aplicada a pares de elementos $\left\{e_{i}, e_{j}\right\} \in E$ a fim de obter um valor de dissimilaridade $d\left(e_{i}, e_{j}\right) \in[0, \infty)$. A construção de $d$ determina a possibilidade de um algoritmo de agrupamento organizar os elementos de $E$ em grupos. Um grupo é essencialmente uma organização de elementos de $E$ em subconjuntos, de modo que cada elemento está contido em um, e somente um, subconjunto ${ }^{2}$. Essa estrutura é conhecida como a partição de um conjunto - partições são denotadas, nesta tese, por letras gregas maiúsculas, tais como $\Gamma$ (Gama) e $\Upsilon$ (Ípsilon). Um algoritmo de agrupamento é, portanto, uma função $f: d \rightarrow \Gamma \in \mathbf{U}$, que mapeia os elementos descritos por uma matriz de distâncias $d$ em uma partição $\Gamma$ de $E$, pertencente ao conjunto universo de partições $\mathbf{U}$.

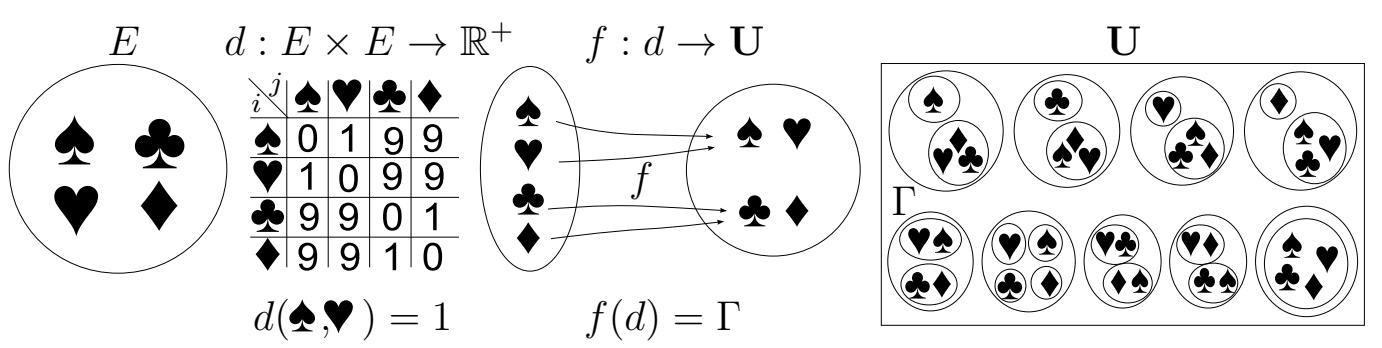

Figura 2.1: Ilustração dos conceitos de agrupamento de dados

Por meio dessa formalização, Kleinberg (2002) propôs propriedades básicas ao problema de agrupamento de dados, introduzidos na forma de declarações de princípios ou condições evidentes para algoritmos de agrupamento respeitarem de acordo com as necessidades do domínio de aplicação. Tendo em vista a descrição de princípios o mais abrangentes possíveis, Kleinberg propôs inicialmente três propriedades: Invariância à Escala (do inglês, Scale-invariance), Completude (Richness) e Consistência (Consistency).

A Propriedade 1, Invariância à Escala, refere-se à capacidade de algoritmos em abstrair a escala para medição de distâncias entre elementos. Essa propriedade reflete a expectativa de que a magnitude da escala de elementos não deve influenciar no particionamento. Isso ocorre, por exemplo, em aplicações cujos objetos são agrupados segundo suas dimensões físicas. Nessas aplicações, é natural esperar que agrupamentos realizados quando dissimilaridades entre objetos são mensuradas em centímetros sejam os mesmos que aqueles quando descritas em polegadas.

Propriedade 1 - Invariância à Escala: Para qualquer função de distância d e qualquer constante $a>0$, vale $f(d)=f(a \cdot d)$.

\footnotetext{
${ }^{2}$ Para agrupamentos nebulosos (em inglês, fuzzy), essa condição é relaxada e um elemento pode pertencer a vários grupos simultaneamente.
} 
Por outro lado, em determinadas situações, o projeto do algoritmo pode requerer a flexibilidade de obter agrupamentos reorganizados de acordo com decisões de especialistas, o que pode levar a abordagem a não respeitar a Propriedade 11. Essa situação é, por exemplo, observada no uso de algoritmos de agrupamento hierárquico em aplicações de mineração de textos, nas quais medidas de comparação não reproduzem a dissimilaridade entre documentos de maneira absoluta e especialistas podem decidir, conforme seu conhecimento sobre a aplicação, o nível de corte de dendrogramas.

A segunda propriedade, Completude, refere-se à capacidade de um algoritmo induzir todas as partições possíveis de qualquer conjunto de elementos. Essa propriedade refere-se a um conceito da Teoria dos Conjuntos denominado sobrejeção (Royden, 1968). Uma função de agrupamento sobrejetora é capaz de relacionar todas as partições em $\mathbf{U}$, o conjunto universo de partições, a partir do domínio, ou seja, o conjunto de elementos E. Essa propriedade assume que o algoritmo pode organizar os elementos-alvo manipulando suas dissimilaridades relativas, a fim de obter qualquer partição provida pelos dados. Nessa propriedade está implícita uma noção subjetiva de que conjuntos podem ter uma organização natural que algoritmos deverão encontrar. Um algoritmo de agrupamento está em conformidade com a Propriedade 2 se, e somente se, ele permitir obter todas as partições para os elementos em $E$.

Propriedade 2 - Completude: Para toda possível partição $\Gamma$ para E, existe uma matriz de distâncias d para a qual $f(d)$ resulta em $\Gamma$.

A garantia da possibilidade de encontrar todas as partições de um conjunto de dados, conforme previsto pela Propriedade 2 , pode parecer, a princípio, desejável para um algoritmo ser aplicado a quaisquer dados cuja natureza é desconhecida. No entanto, por sua generalidade, essa propriedade pode ser difícil de ser cumprida (Kleinberg, 2002).

A Propriedade 3, ou seja, Consistência, refere-se à capacidade de algoritmos manterem a mesma partição quando distâncias entre elementos do mesmo grupo são reduzidas e distâncias entre elementos de diferentes grupos são aumentadas.

Propriedade 3 - Consistência: Considere uma partição $\Gamma$ obtida por uma função de agrupamento $f(d)$. Considere d', uma modificação da matriz de distâncias d de forma que aumente, ou mantenha, as distâncias entre elementos de subconjuntos distintos de $\Gamma$ e que reduza, ou mantenha, as distâncias entre elementos do mesmo subconjunto. Uma função de agrupamento que respeita esta propriedade é tal que $f\left(d^{\prime}\right)=\Gamma$.

A importância das propriedades previamente descritos reside tanto no campo teórico, conforme demonstrado em um teorema de impossibilidade em Kleinberg (2002), quanto no campo prático, na compreensão profunda do problema de agrupamento de dados e proposta de novos algoritmos (Carlsson \& Mémoli, 2008; Carlsson \& Mémoli, 2010a; Zadeh, 2010). 
Porém, de acordo com os estudos realizados no contexto desta tese, não existe formalização equivalente para o problema de agrupamento de fluxos de dados (von Luxburg \& Schoelkopf, 2008). No entanto, vale ressaltar o estudo realizado por Ryabko (2010), que considera o problema de agrupamento de processos estocásticos estacionários. Nele, cada grupo é associado a um processo estocástico com distribuição de probabilidade constante ao longo do tempo. Contudo, a aplicabilidade desse estudo é limitada no contexto dos fluxos de dados, no qual são esperadas variações comportamentais ao longo do tempo.

\subsection{O problema de agrupamento de fluxos de dados}

Fluxos de dados diferem de outros tipos de conjuntos tradicionalmente considerados em métodos de agrupamento. Eles são sequências ilimitadas coletadas a partir da observação de fenômenos com comportamento desconhecido ou instável (Guha et al., 2003). Essas características contrastam com conjuntos tradicionais de dados, que são finitos, não possuem uma ordem particular e apresentam estabilidade. Essas distinções motivam a adaptação das propriedades de Kleinberg (2002), que não consideram a natureza sequencial do problema de agrupamento de fluxos de dados.

Um fluxo de dados é representado por um conjunto infinito e ordenado de elementos, também conhecidos como padrões de entrada, (Sousa \& Traina, 2006; Sun \& Lu, 2006). Um fluxo é denotado por uma sequência $S=\{s(-\infty), \ldots, s(t), \ldots, s(\infty)\}$, em que $t \in \mathbb{Z}$ representa o instante de tempo de coleta ou ordem de processamento de $s(t)$. Dessa forma, o padrão de entrada $s(0)$ é o elemento mais recentemente coletado e os elementos seguintes representam aqueles que serão futuramente coletados.

Enquanto a sequência completa é representada por uma letra maiúscula $S$, uma subsequência de elementos coletados até o instante de tempo $t$ é denotada $S(t)$. Cada elemento $s(t) \in S(t)$ nessa sequência consiste de um vetor de $l$ atributos mensurados em $\mathbb{R}$. Elementos em $S(t)$ são comparados por meio de uma função de dissimilaridade $d: S \times S \rightarrow \mathbb{R}^{+}$.

O objetivo de um algoritmo de agrupamento de fluxo de dados $f(d, S(t))$ é produzir uma sequência de partições $\bar{\Gamma}(0)=\{\Gamma(-\infty), \ldots, \Gamma(0)\}$ para os elementos em $S(0)$, em que $\Gamma(0)=f(d, S(0))$. Essa sequência contém todas as partições produzidas desde $S(-\infty)$ até $S(0)$, ou seja, desde que o primeiro elemento do fluxo de dados foi recebido pelo algoritmo de agrupamento até o mais recente ${ }^{3}$.

A partir dessa definição, ilustrada na Figura 2.2, a principal diferença do problema de agrupamento de fluxos de dados, quando comparado ao agrupamento de conjuntos tradicionais de dados, advém de sua natureza infinita e ordenada, o que exige a obtenção de uma sequên-

\footnotetext{
${ }^{3}$ Ressalta-se que, embora $S$ seja infinito, partições em $\bar{\Gamma}$ devem ter um número finito de subconjuntos disjuntos (sem elementos em comum).
} 


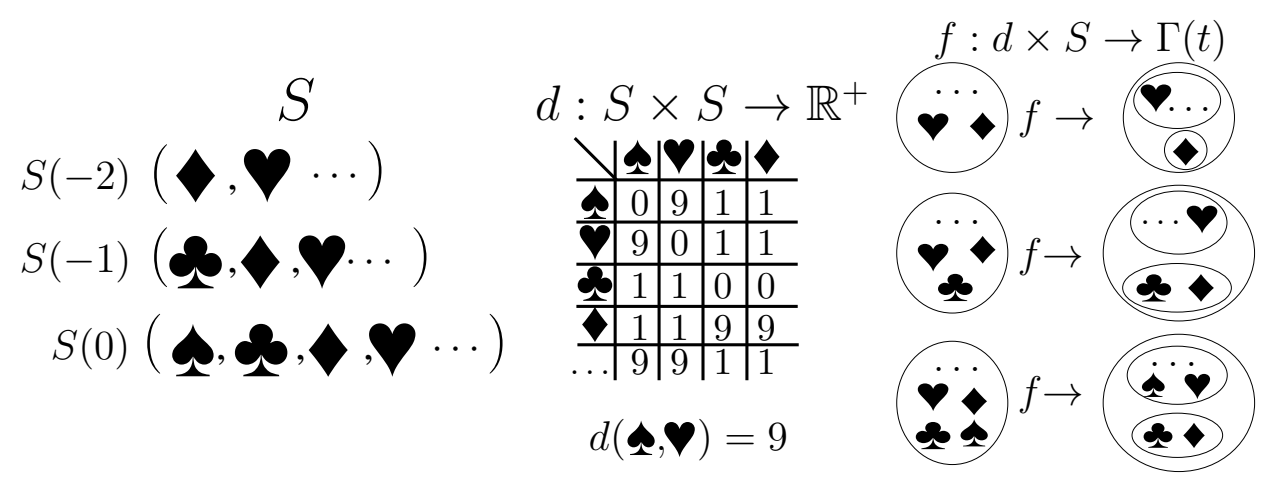

Figura 2.2: Ilustração dos conceitos de agrupamento de fluxos de dados

cia de partições ao longo do tempo, em vez de uma única partição. Essas particularidades motivaram a adaptação das propriedades de Kleinberg para o contexto de fluxos de dados. Portanto, propõe-se, no contexto desta tese, as seguintes propriedades para o problema de agrupamento de fluxos de dados: Invariância Espaço-temporal, Completude em Fluxos de Dados e Consistência Espaço-temporal.

A Propriedade 4, denominada Invariância Espaço-temporal, é equivalente à Propriedade 1 proposta por Kleinberg (2002). Essa propriedade exige que um algoritmo produza a mesma partição caso as escalas de medida de tempo ou espaço sejam transformadas pela multiplicação por constantes. Essa propriedade é útil em aplicações em que a escala de medição do instante de tempo da coleta de amostras não deve influenciar nas partições produzidas.

Propriedade 4 - Invariância Espaço-temporal: Considere a multiplicação dos índices de tempo de cada elemento $s \in S(t)$ por uma constante a e d por uma outra constante $b$, a sequência de partições $\bar{\Gamma}(t)$ produzida por $f(b \cdot d, s(a \cdot t))$ não se altera.

De modo similar, as propriedades de Completude em fluxos de dados e Consistência Espaço-temporal são adaptações de Completude e Consistência para conjuntos tradicionais de dados. Ambas propriedades incluem uma noção de proximidade temporal e, portanto, distinguem-se por definir um ponto de referência no tempo para conduzir comparações entre partições.

A Propriedade 5, que define a Completude em fluxos de dados, estabelece que um algoritmo de agrupamento pode obter todas as combinações de grupos para os elementos coletados, considerando, inclusive diferentes ordens de coleta. Para fluxos de dados, cujo comportamento é variável, é interessante que algoritmos de agrupamento não sejam limitados a produzir apenas certos tipos de partições.

Propriedade 5 - Completude em fluxos de dados: Considere um ponto de referência no instante de tempo atual, i.e., 0, uma sequência $S(0)=\{s(-\infty), \ldots, s(0)\}$, e uma matriz de dissimilaridades $d$ entre elementos em $S(0)$. Uma função de agrupamento $f$ respeita a 
propriedade de Completude em fluxos de dados no instante atual, i.e., 0, se ela for capaz de obter todas as possíveis sequências de partições para $S(0)$, considerando uma transformação arbitrária de de da ordem de coleta dos elementos em $S(0)$.

A Propriedade Consistência Espaço-temporal estende a Propriedade 3 ao incluir a noção de proximidade temporal. Essa propriedade afirma que se elementos do mesmo grupo são modificados de modo que se tornem temporal e espacialmente mais próximos e elementos de diferentes grupos, mais separados, então a sequência de partições deve ser mantida.

Propriedade 6 - Consistência Espaço-temporal: Considere d' e $S^{\prime}(t)$, transformações da matriz de dissimilaridades de da sequência de dados $S(t)$, tais que os intervalos e distâncias de ocorrência entre elementos de um agrupamento se tornem menores e entre grupos diferentes, maiores. Uma função de agrupamento f respeita esta propriedade se as sequências de partições obtidas em ambos os casos são iguais, i.e., $f(d, S(t))=f\left(d^{\prime}, S^{\prime}(t)\right)$.

As propriedades previamente propostas são extensões das definidas para o problema de agrupamento de conjuntos tradicionais de dados. Além dessas propriedades, são necessárias condições mínimas para garantir que um algoritmo de agrupamento sempre obtenha sequências de partições que representem o comportamento do fenômeno em estudo. $\mathrm{Na}$ prática, deseja-se que um algoritmo atribua elementos a grupos de modo coerente ao longo das partições obtidas. Por exemplo, em uma aplicação na qual protótipos dos grupos variam continuamente (Rodrigues et al., 2008), a avaliação de agrupamentos no tempo é necessária. Entretanto, caso o algoritmo não produzir partições consecutivas relacionadas, a comparação entre essas pode ser inválida e, portanto, o estudo de variações de comportamento de fenômenos pode ser comprometido.

Observou-se, entretanto, que as propriedades previamente definidas não garantem que sequências de agrupamento evoluam com continuidade ao longo do tempo, a fim de representar o comportamento do fenômeno. O conceito de continuidade entre partições é formalizado (Definição 2) por uma relação denominada alcançar, representada por $\Gamma \triangleright \Upsilon$, de acordo com a Definição 1. A relação alcançar é inspirada em uma aplicação do conceito de refinamento de Kleinberg (2002). Uma partição $\Gamma$ é um refinamento de outra $\Gamma^{\prime}$ se todos os subconjuntos em $\Gamma$ estão contidos em subconjuntos de $\Gamma^{\prime}$. Dessa forma, $\Gamma$ alcança $\Upsilon$ se $\Gamma$ for um refinamento de $\Upsilon$ ou vice-versa, excluindo os elementos que não pertencem a ambas partições.

Definição 1 - Alcançar: Uma partição $\Gamma$ alcança outra partição $\Upsilon$ se, para cada subconjunto $A \in \Gamma$, existe outro subconjunto $B \in \Upsilon$ tal que $\left(A \backslash R^{\Gamma}\right) \subseteq\left(B \backslash R^{\Upsilon}\right)$ ou $\left(B \backslash R^{\Upsilon}\right) \subseteq\left(A \backslash R^{\Gamma}\right)$, dado que a operação \é definida por $B \backslash A=\{s \in B \mid s \notin A\}$ e que $R^{\Gamma}$ é o subconjunto de elementos pertencentes a $\Gamma$ e não a $\Upsilon$, e $R^{\Upsilon}$ é o subconjunto de elementos pertencentes a $\Upsilon$ não a $\Gamma$. A relação alcançar é denotada por $\Gamma \triangleright \Upsilon$. 
Por exemplo, considerando $\Gamma=\{\{1,2\},\{3\}\}$ e $\Upsilon=\{\{2,3\},\{4\}\}$, para verificar se vale a relação $\Gamma \triangleright \Upsilon$ deve-se observar que $R^{\Gamma}=\{1\}$ e $R^{\Upsilon}=\{4\}$ e que para cada $A \in \Gamma$ existe $B \in \Upsilon$, tal que $A \subseteq B$. Dessa forma, tomando $A=\{2\}$ ou $A=\{3\}$, tem-se $A \subset B$ pois $B=\{2,3\}$, o que confirma que a relação $\Gamma \triangleright \Upsilon$ é válida.

Uma sequência de partições é contínua, segundo a Definição 2, quando a relação alcançar valer para todo par de partições consecutivas.

Definição 2 - Continuidade de uma sequência de partições: Uma sequência de partições $\bar{\Gamma}(t)$ é contínua se, e somente se, para todos os pares de partições consecutivas, i.e., $\Gamma(t-i-1) e$ $\Gamma(t-i)$, na qual $i \geq 0$, a relação $\Gamma(t-i-1) \triangleright \Gamma(t-i)$ é verdadeira.

A definição da continuidade de uma sequência de partições motiva a proposta da propriedade de Coerência (Propriedade 7), que estabelece que um algoritmo coerente de agrupamento de fluxo de dados deve somente obter sequências de partições contínuas, nas quais grupos apenas se alteram respeitando a relação de alcance, ou seja, a continuidade dos agrupamentos obtidos ao longo do tempo.

Propriedade 7 - Coerência: Para qualquer $d, S(t)$ e $\Gamma(t)=f(d, S(t))$ a sequência de partições $\bar{\Gamma}(t)$ é contínua.

A relevância da Coerência, conforme estabelecida na Propriedade 7 para algoritmos de agrupamento de fluxos de dados reside no paralelo que se pode traçar entre a continuidade de agrupamentos e de funções. Um algoritmo de agrupamento coerente garante a produção de sequências de partições contínuas, o que permite o uso de medidas de avaliação de qualidade aplicadas ao longo da coleta dessa sequência. Por exemplo, o uso do algoritmo K-médias (Hartigan, 1975) para agrupar fluxos a partir de estruturas de microgrupos, tal como realizado pelos algoritmos BIRCH (Zhang et al., 1996) e CLUSTREAM (Aggarwal et al., 2003), não garantirá a continuidade das partições, devido à sua natureza aleatória na inicialização de protótipos, o que pode levar o algoritmo K-médias a produzir partições consecutivas que não respeitam a relação alcançar entre si. Dessa forma, sem a garantia de Coerência é possível a obtenção de valores espúrios na aplicação de medidas que avaliam variações entre partições subsequentes, pois essas podem não estar relacionadas (Capítulo 3). Uma aplicação dessa propriedade encontra-se na caracterização do comportamento de fenômenos, em que se compara partições consecutivas a fim de obter medidas que caracterizam variações de comportamento (Gamon, 2006; Mello et al., 2010).

\subsubsection{Análise e projeto de agrupamento para fluxos de dados}

A formalização previamente apresentada foi proposta nesta tese com o intuito de aprofundar na compreensão do problema de agrupamento de fluxos de dados. Ela auxilia no 
projeto, análise e comparação de algoritmos de agrupamento de fluxos de dados ao fornecer uma base teórica para a verificação de requisitos de agrupamento de acordo com o fenômeno estudado. Por exemplo, Kleinberg (2002) provou a inexistência de um algoritmo de agrupamento tradicional que atende simultaneamente suas Propriedades 1, 2, e 3 .

É possível observar uma condição de impossibilidade análoga na obtenção de um algoritmo de agrupamento de fluxos de dados para as três propriedades adaptadas. Além disso, as Propriedades 5 e 7 são mutuamente exclusivas. Para demonstrá-lo, deve-se provar que se uma função de agrupamento de fluxos $f$ respeita Completude, então ela necessariamente cria sequências de partições $\bar{\Gamma}$ nas quais pelo menos um par de partições consecutivas não respeita a relação alcançar; e, também, se $f$ cria somente sequências de partições contínuas, então ela não respeita a propriedade de Completude. O Teorema 1 demonstra a impossibilidade de projetar um algoritmo de agrupamento que atenda, simultaneamente, as propriedades de Coerência e Completude.

Teorema 1 Não existe uma função de agrupamento de fluxos de dados $f$ que, simultaneamente, respeite as Propriedades 5 i.e., Completude em fluxos de dados, e 7 i.e., Coerência.

Prova: Primeira parte: assuma que $f$ respeita Completude em fluxos de dados, então $f$ produz uma sequência de partições $\bar{\Gamma}$ contendo partições consecutivas que não se alcançam.

Provar-se-á que existe uma sequência $\bar{\Gamma}$ com partições consecutivas que não se alcançam, independentemente de manipulações na matriz de distâncias $d$ e na sequência de elementos $S(t)$. Uma partição $\Gamma(t)$ é inalcançável quando não existe uma partição $\Gamma(t-1)$ que a precede tal que a relação $\Gamma(t-1) \triangleright \Gamma(t)$ é respeitada. Isso é, o conjunto de todas as possíveis partições no instante $t-1$ é vazio. Note, portanto, que basta um exemplo para provar que existe uma partição $\Gamma(t)$ inalcançável.

Assuma uma partição no instante de tempo $t$

$$
\Gamma(t)=\left\{\left\{a_{1}, b_{2}\right\},\left\{a_{2}, b_{1}\right\}\right\}
$$

em que, elementos $a_{i}, \forall i$ e $b_{j}, \forall j$ em

$$
\Gamma(t-1)=\left\{\left\{a_{1}, a_{2}\right\},\left\{b_{1}, b_{2}\right\}\right\}
$$

estão em grupos diferentes.

Sabe-se, contudo, que $\left\{\left\{a_{1}, a_{2}\right\},\left\{b_{1}, b_{2}\right\}\right\} \triangleright\left\{\left\{a_{1}, b_{2}\right\},\left\{a_{2}, b_{1}\right\}\right\}$ não é válido porque, de acordo com a definição da relação alcançar, nenhum subconjunto da primeira partição está contido em um subconjunto da segunda, e vice-versa. Também se sabe que, por definição, qualquer $f$ que respeita Completude para fluxos de dados deve produzir tal sequência de 
partições.

Segunda parte: se $f$ produz somente sequências de partições contínuas, então $f$ não respeita Completude para fluxos de dados.

Considerando a partição $\Gamma(t-1)=\left\{\left\{a_{1}, a_{2}\right\},\left\{b_{1}, b_{2}\right\}\right\}$, então se sabe que $f$ não é capaz de produzir, no instante de tempo consecutivo $t$,

$$
\Gamma(t)=\left\{\left\{a_{1}, b_{2}\right\},\left\{b_{1}, a_{2}\right\}\right\}
$$

e, de modo similar, considerando

$$
\Gamma(t-1)=\left\{\left\{a_{1}, b_{2}\right\},\left\{b_{1}, a_{2}\right\}\right\},
$$

então $f$ não produzirá

$$
\Gamma(t)=\left\{\left\{a_{1}, a_{2}\right\},\left\{b_{1}, b_{2}\right\}\right\}
$$

Portanto, nenhum algoritmo $f$ que produz sequências de partições contínuas pode respeitar a Propriedade 5 .

Pode-se observar que, atualmente, muito pouco é conhecido sobre as fundações teóricas de agrupamento de dados (Ben-David et al., 2006; Carlsson \& Mémoli, 2010b). Por exemplo, salvo poucas exceções, desconhece-se (Ben-David et al., 2006) quais algoritmos respeitam cada propriedade, um bom conjunto de propriedades para orientar especialistas na escolha de algoritmos, ou como projetar um bom algoritmo de agrupamento de acordo com as características do fenômeno estudado. Esse conhecimento é ainda mais reduzido quando se trata de fluxos de dados.

Acredita-se, portanto, que a formalização introduzida nesta tese auxiliará em pesquisas sobre como associar as características de fenômenos à escolha de propriedades. A definição de propriedades e suas relações com o comportamento presente em fluxos de dados e o conhecimento da compatibilidade entre propriedades pode auxiliar na compreensão dos limites teóricos e requisitos para o estudo de fenômenos complexos, tal como foi avaliado no Teorema 1 sobre a incompatibilidade entre as Propriedades 5] (7) Também se espera que, futuramente, tais propriedades façam parte da adaptação de algoritmos de agrupamento conforme variações comportamentais presentes em fluxos de dados. 


\subsection{Algoritmos de agrupamento de fluxos de dados}

Algoritmos de agrupamento de fluxos de dados podem ser organizados em duas categorias. A primeira categoria busca reduzir fluxos a conjuntos finitos e não sequenciais com o objetivo de manipulá-los como conjuntos tradicionais de dados. Pesquisas relacionadas a essa primeira categoria têm se concentrado em prover algoritmos cuja execução seja de baixa complexidade de tempo e espaço ( $\mathrm{Xu} \&$ Wunsch, 2005). A segunda categoria tem o intuito de processar fluxos de dados conforme são produzidos, ou seja, em sequência, sem demais simplificações e perda de informação. Trabalhos dedicados a essa categoria têm concentrado esforços na compreensão de fluxos de dados por meio da adaptação de agrupamentos de acordo com variações comportamentais do fenômeno em estudo.

Em um dos trabalhos mais influentes da primeira categoria, Zhang et al. (1996) propuseram uma estrutura de processamento eficiente de dados para representar grupos de baixo nível, conhecidos como microgrupos, denominada Concept Feature Tree. Essa estrutura é similar a uma Árvore B e foi utilizada em um algoritmo denominado BIRCH com o objetivo de obter complexidade de tempo linear no acesso e modificação de microgrupos. Além disso, ela permite a aproximação de grupos tradicionais por meio de estatísticas-resumo para a média e dispersão de padrões. Após a etapa de microagrupamento, que reduz o número de elementos a serem considerados, aplica-se um algoritmo de agrupamento tradicional tal como o K-médias.

Essa mesma estratégia de microagrupamento influenciou uma geração de algoritmos de agrupamento de fluxos de dados, tais como: STING (Wang et al., 1997), WAVECLUSTER (Sheikholeslami et al., 1998), OPTIGRID (Hinneburg \& Keim, 1999) e CLUSTREAM (Aggarwal et al., 2003). Além do uso de estruturas de microagrupamento, o projeto desses algoritmos buscou adequar a complexidade de tempo e espaço ao processar fluxos de dados por meio de diversas técnicas, tais como amostragem dos dados (STING e CURE), transformação em espaço de frequências (WAVECLUSTER), cálculo de projeções (OPTIGRID) e hierarquias de estruturas de microagrupamento (CLUSTREAM).

STING (Wang et al., 1997; Yu et al., 2010) é um algoritmo de baixa complexidade computacional baseado em uma estrutura hierárquica cujas folhas representam subespaços de dados, ou seja, constituem uma grade em que distribuições de probabilidades são estimadas. Essas folhas formam microgrupos, que são posteriormente processados por um algoritmo de agrupamento tradicional.

Similarmente, o algoritmo WAVECLUSTER (Sheikholeslami et al., 1998) baseia-se em uma estrutura de grade, porém os dados originais são projetados em um espaço de frequências por meio de uma transformada Wavelet. Essa transformada decompõe os dados em faixas de frequências com diferentes escalas de resolução, o que garante que a região dos elementos com maior frequência, isso é, as bordas de grupos, serão melhor observados. Entretanto, o WAVECLUSTER desconsidera a ordem de coleta de dados, o que o torna insensível a variações 
de comportamento.

OPTIGRID (Hinneburg \& Keim, 1999) é especializado em fenômenos que necessitam da análise simultânea de um grande número de atributos. Nesses fenômenos, a principal dificuldade encontra-se no uso de funções de proximidade, tal como a distância euclidiana, que potencializam a influência de ruídos e comprometem a definição de grupos. Para contornar essa dificuldade, o OPTIGRID utiliza uma abordagem partitiva para obter um menor número de atributos dos dados para definir grupos. Contudo, apesar da eficiência desse algoritmo, sua estrutura recursiva para refinar planos que dividem dados e encontram grupos não permite sua aplicação incremental em fluxos de dados.

Diferentemente dessas técnicas anteriormente apresentadas, Aggarwal et al. (2003) dedicaram-se ao estudo de fluxos de dados com variação de comportamento mediante o emprego de microagrupamentos. Eles propõem uma técnica denominada CluSTREAM que emprega uma estrutura piramidal de Concept Feature Tree com o objetivo de organizar as informações sobre alterações dos dados em diferentes horizontes de tempo. Essa técnica também requer uma etapa de processamento baseada no algoritmo K-médias, na qual o especialista deve conhecer o número de grupos relevantes. Dessa forma, a compreensão do comportamento do fenômeno em estudo depende de parâmetros que são mantidos constantes, o que inclui a definição de horizontes de tempo relevantes e o número de grupos. O CLUSTREAM, assim como outras técnicas anteriormente mencionadas, não está preparado para cenários em que o comportamento observado durante a etapa de projeto do algoritmo de agrupamento e determinação de seus parâmetros varie significativamente.

A segunda categoria de algoritmos de agrupamento processa dados por meio da adaptação de grupos em resposta direta a alterações no comportamento observadas em fluxos de dados. Diversos algoritmos do segundo tipo são conhecidos (Carpenter et al., 1991; Fritzke, 1995; Biehl et al., 2006; Young et al., 2010), os quais são frequentemente baseados em redes neurais artificiais não supervisionadas e em versões incrementais de algoritmos de agrupamento. Dentre os algoritmos baseados em redes neurais artificiais pode-se mencionar: SelfOrganizing Maps (Kohonen, 1997), Recurrent Self-Organizing Maps e Grow When Required (Marsland, 2002). Dentre os algoritmos de agrupamento incrementais estão o algoritmo Growing K-médias (Daszykowski et al., 2002), a abordagem OlindDA (Spinosa et al., 2007) e o algoritmo Sequential Leader Clustering (Hartigan, 1975).

A arquitetura de rede neural artificial Self-Organizing Maps (Kohonen, 1997) (SOM) é um método tradicional de aprendizado não supervisionado baseado em modelos associativos, no qual neurônios respondem a estímulos treinando o protótipo mais adequado. Uma rede SOM é composta por um número fixo de neurônios, representados por protótipos, que são vetores numéricos de mesma dimensionalidade que os padrões de entrada. O objetivo da etapa de treinamento de uma rede $\underline{\mathrm{SOM}}$ é obter protótipos representativos para esses padrões. Durante essa etapa, o protótipo do neurônio que responde mais fortemente ao estímulo de um 
vetor de entrada, aproxima-se desse vetor de acordo com uma taxa de aprendizado $\eta \geq 0$. Portanto, o treinamento da rede SoM utiliza a Equação 2.1 para atualizar, de acordo com uma taxa de aprendizado $\eta \in \mathbb{R}$, o protótipo $\vec{w}_{\mathrm{BMU}}(t)$ do neurônio Best-Matching Unit (BMU), o qual apresentou a resposta mais forte em relação ao vetor de entrada $\vec{I}(t)$ no instante de tempo t. Os neurônios vizinhos ao BMU atualizam seus protótipos de acordo com uma função de proximidade de vizinhança $\Theta_{\mathrm{BMU}}$, na forma $\Theta_{\mathrm{BMU}} \cdot \eta \cdot\left(\vec{I}(t)-\vec{w}_{\mathrm{BMU}}(t-1)\right)$.

$$
\vec{w}_{\mathrm{BMU}}(t)=\vec{w}_{\mathrm{BMU}}(t-1)+\eta \cdot\left(\vec{I}(t)-\vec{w}_{\mathrm{BMU}}(t-1)\right)
$$

A arquitetura $\widehat{S O M}$ tem sido utilizada como modelo para algoritmos de agrupamento de fluxos de dados. Por exemplo, a arquitetura Recurrent Self-Organizing Maps (RSOM (Varsta et al., 1997) acopla ao treinamento de protótipos da SoM um fator de memória no aprendizado de estímulos. Esse fator de memória de padrões $\vec{m}_{\mathrm{BMU}}(t)$ é definido na Equação 2.2 de acordo com um coeficiente de esquecimento $\varphi \in(0,1]$. Quanto maior o valor de $\varphi$, menor é a influência de padrões passados no treinamento. A equação de atualização de um protótipo $\vec{w}_{\mathrm{BMU}}(t)$ é estabelecida como $\vec{w}_{\mathrm{BMU}}(t)=\vec{w}_{\mathrm{BMU}}(t-1)+\Theta_{\mathrm{BMU}} \cdot \eta \cdot \vec{m}_{\mathrm{BMU}}(t)$. Devido à manutenção de uma memória, a rede $\mathrm{RSOM}$ apresenta a capacidade de aprender sobre o contexto sequencial dos dados. Porém o parâmetro que controla tal memória é mantido constante, independentemente de variações de comportamento observadas em dados, o que limita o estudo de fenômenos.

$$
\vec{m}_{\mathrm{BMU}}(t)=\vec{m}_{\mathrm{BMU}}(t) \cdot(1-\varphi)+\varphi\left(\vec{I}(t)-\vec{w}_{\mathrm{BMU}}(t-1)\right)
$$

Outros estudos buscaram permitir que redes modifiquem, ao longo do processo de aprendizado, a estrutura do mapa de seus neurônios. Marsland (2002), em sua tese de doutorado, realizou um levantamento de técnicas que adicionam neurônios para alterar a forma do mapa auto-organizável conforme a necessidade de representação dos dados. Entre essas técnicas estão Reduced Coulomb Energy (Reilly et al., 1982), Neural Gas (Martinetz \& Schulten, 1994) e Growing Neural Gas (Fritzke, 1995). Essas técnicas apresentam em comum a necessidade de definir uma regra que determina o momento de adição de novos neurônios. Contudo, Marsland (2002) observou que as regras propostas não orientam adequadamente a ocorrência de modificações, que devem ser realizadas em momentos de significativa variação de comportamento nos dados.

A partir dessa observação, o algoritmo Grow When Required (GWR) foi proposto, o qual segue um modelo diferencial baseado em um fenômeno biológico denominado habituação (Stanley, 1976). Esse fenômeno consiste na atenuação da resposta de neurônios a estímulos frequentes e indica o amadurecimento de seu aprendizado. A habituação foi utilizada para indicar variações de comportamento entre padrões observados, ou seja, caso o neurônio com 
maior resposta esteja com um alto nível de habituação e o vetor de entrada tenha caído fora de um limiar de distância mínimo, então um novo neurônio deve ser criado, caso contrário o neurônio BMU deve ser adaptado.

Esses algoritmos de aprendizado auto-organizável utilizados em agrupamento de fluxos de dados inspiram-se em abordagens utilizadas em agrupamento incremental, sem fundamentação biológica. Algoritmos incrementais de agrupamento visam adicionar novos padrões de entrada a grupos sem a reavaliação de dados prévios (Marsland, 2002; Itti \& Baldi, 2005). Devido à baixa complexidade computacional, essas técnicas apresentam um grande potencial para aplicação em fluxos de dados. Abordagens baseadas em algoritmos de agrupamento incremental, tais como os algoritmos Sequential Leader Clustering (Hartigan, 1975) e Growing K-médias (Daszykowski et al., 2002), buscam representar padrões de entrada modificando os protótipos mais representativos, ou, caso os padrões se diferenciam significantemente dos protótipos atuais, adicionando gradualmente novos grupos. O algoritmo Growing K-médias utiliza uma regra de adaptação do protótipo na forma da Equação 2.1, sem criar novos grupos. Por outro lado, no algoritmo Sequential Leader Clustering, a pertinência de um novo vetor de entrada a grupos é verificada por meio do uso de um limiar estático. Caso esse vetor pertença a um grupo, nada é realizado, caso contrário, um novo grupo é criado tendo como protótipo o próprio vetor de entrada.

Outras abordagens mais sofisticadas, tais como a abordagem OLINDDA (Spinosa et al., 2007), incluem mecanismos explícitos para a caracterização de comportamento normal e modificação dos parâmetros de agrupamento. A abordagem OLINDDA utiliza uma etapa inicial de agrupamento em que um algoritmo K-médias estabelece uma partição de referência, denominada comportamento normal. Posteriormente, durante a etapa de agrupamento de fluxos de dados, esse comportamento normal é ajustado. Novos grupos no modelo normal podem ser definidos ao longo do tempo de acordo com um parâmetro que impõe um limiar mínimo de número de elementos por grupo. Adicionalmente, caso um conjunto de elementos forme um grupo ausente no modelo normal, um novo grupo pode ser criado.

Young et al. (2010) propuseram um algoritmo baseado na abordagem Winner Takes All, denominado Starvation WTA, equivalente a redes neurais auto-organizáveis cujo grupo escolhido para representar o padrão de entrada, ou seja, o neurônio BMU, não possui vizinhos para propagar atualizações. Essa abordagem orienta o processo de agrupamento a partir de uma medida de Starvation (em português, privação), que aumenta a probabilidade e a taxa de treinamento de protótipos menos representativos. O uso dessa medida permite a adaptação de grupos quando eles se tornam inadequados devido a variações de comportamento.

Contudo, mesmo algoritmos mais recentes como Starvation WTA e OLINDDA não chegaram ao ponto de definir como objetivo a adaptação do viés de parâmetros de aprendizado durante o processo de agrupamento. Eles reconhecem variações de comportamento, adequam os agrupamentos existentes, ou adicionam novos grupos, porém mantêm o viés indutivo está- 
tico.

Em resumo, algoritmos de agrupamento para fluxos de dados apresentam limitações para reconhecer variações de comportamento e adaptar parâmetros que influenciam o viés indutivo. As características de falta de adaptabilidade do CLUSTREAM são comuns à maior parte das técnicas da primeira categoria de algoritmos de agrupamento de fluxos de dados, que os consideram como grandes conjuntos de dados estáticos. Além disso, a maior parte dos algoritmos do primeiro tipo, i.e., que reduzem fluxos de dados a conjuntos finitos, não são adequados para reconhecer variações de comportamento, enquanto algoritmos do segundo tipo, tais como GWR e OLINDDA, i.e., que processam diretamente fluxos de dados, podem reconhecer, mas não são preparados para prover adaptação do viés indutivo em cenários de variação de comportamento.

\subsubsection{Análise comparativa de algoritmos de agrupamento de fluxos de dados}

Com o objetivo de prover uma perspectiva formal para a compreensão de algoritmos de agrupamento de fluxos de dados, assim como foi realizado por Kleinberg (2002), uma definição formal do problema foi proposta por meio de um arcabouço teórico baseado em propriedades e conceitos de Teoria dos Conjuntos.

O uso de propriedades básicas para a análise de algoritmos de agrupamento é incipiente. Porém, elas estabelecem condições básicas, as quais provêm ferramentas úteis para a compreensão aprofundada de problemas (Ben-David \& Ackerman, 2009). O estudo de agrupamento de fluxos de dados a partir de propriedades básicas permite a compreensão de princípios teóricos de algoritmos (Ben-David \& Ackerman, 2009) e o projeto e a seleção de algoritmos por meio da escolha de propriedades levando em conta fatores utilitários e econômicos inerentes ao domínio da aplicação (Antzoulatos \& Vrahatis, 2011).

Especula-se que, no futuro, essa formalização deva permitir o acúmulo de conhecimento genérico o suficiente sobre o problema de agrupamento e auxilie na composição de uma Teoria de Agrupamento em busca da unificação da literatura atual (Meila, 2005; Antzoulatos \& Vrahatis, 2011).

As propriedades propostas na Seção 2.4 são as primeiras que representam as características inerentes a fluxos de dados. Essas propriedades são: Propriedade 4, Invariância Espaço-temporal; Propriedade 5, Completude em Fluxos de Dados; Propriedade 6, Consistência Temporal; e Propriedade 7, Coerência. A partir dessas propriedades, é possível realizar uma comparação dos algoritmos de agrupamento de fluxos de dados atualmente mais relevantes. A comparação é resumida ${ }^{4}$ na Tabela 2.2 , na qual foram selecionados algorit-

\footnotetext{
${ }^{4} \mathrm{O}$ símbolo ‘- ' representa que não foi possível obter a respectiva avaliação.
} 
mos que apresentam características marcantes: BIRCH (Zhang et al., 1996), WAVECLUSTER (Sheikholeslami et al., 1998), CLUSTREAM (Aggarwal et al., 2003), OLINDDA (Spinosa et al., 2007) e Starvation WTA (Young et al., 2010).

Tabela 2.2: Avaliação das propriedades de algoritmos de agrupamento de fluxos de dados

\begin{tabular}{ccccc}
\hline Algoritmo & Invariância & Completude & Consistência & Coerência \\
\hline BIRCH (1996) & $\mathrm{N}$ & $\mathrm{N}$ & $\mathrm{N}$ & $\mathrm{N}$ \\
WaveCluster (1998) & $\mathrm{N}$ & $\mathrm{N}$ & - & $\mathrm{N}$ \\
CluStream (2003) & $\mathrm{N}$ & $\mathrm{N}$ & $\mathrm{N}$ & $\mathrm{N}$ \\
OLINDDA (2007) & $\mathrm{S}$ & $\mathrm{N}$ & $\mathrm{N}$ & $\mathrm{N}$ \\
WTA (2010) & $\mathrm{S}$ & $\mathrm{N}$ & $\mathrm{S}$ & $\mathrm{S}$ \\
\hline
\end{tabular}

Usualmente, para verificar se algoritmos respeitam tais propriedades, pode-se provar um teorema ou apresentar um contraexemplo. Contudo, em alguns casos não é possível realizar nenhuma das duas opções. Contudo, existem outras opções para verificar se um algoritmo respeita uma dada propriedade. Por exemplo, sabe-se que, caso o algoritmo limite o número de grupos, então ele não respeita a propriedade de Completude (Kleinberg, 2002). Também se sabe que um algoritmo não respeita a Propriedade 4, i.e., Invariância Espaço-temporal, caso utilize um limiar de aceitação de elementos em grupos. Isso porque, para qualquer limiar de aceitação estabelecido, existe uma constante para mudança de escala dos dados que fará com que elementos mudem de grupos. Um algoritmo também não respeita a Propriedade 4 e a Propriedade 7, i.e., Coerência, caso não considere a ordem dos dados para agrupamento.

Uma outra opção para esse tipo de análise é utilizar as provas previamente estabelecidas para algoritmos tradicionais como K-médias e algoritmos hierárquicos Single-linkage em algoritmos que utilizam microagrupamento para identificar o atendimento às propriedades de fluxos de dados (Aggarwal et al., 2003; Zhang et al., 1996). Portanto, algoritmos que utilizam K-médias não respeitam a Propriedade 7 pois, apesar da inserção de um elemento em um microagrupamento poder causar apenas uma junção ou separação de grupos, a utilização do algoritmo K-médias para obtenção de agrupamento não garante sua continuidade.

\subsection{Considerações finais}

Neste capítulo, introduziu-se o problema de agrupamento de fluxos de dados de maneira formal utilizando elementos da Teoria dos Conjuntos. Essa formalização, proposta nesta tese, apresenta propriedades para algoritmos de agrupamento de fluxos de dados, as quais foram utilizadas para analisá-los. A partir dessa análise, pôde-se verificar que, atualmente, algoritmos de agrupamento não estão preparados para a adaptação automática de viés indutivo por meio de parâmetros de acordo com variações de comportamento presentes em fluxos de dados. Para isso seria necessário que esses algoritmos respeitassem a Propriedade de 
Coerência, com o objetivo de garantir a correta avaliação da variação de comportamento entre partições consecutivas. Vale ressaltar a importância de formalizações tal como a proposta para auxiliar no acúmulo de conhecimento, possibilitar a análise comparativa da literatura atual e orientar o projeto e desenvolvimento de novos algoritmos dedicados à compreensão de fenômenos complexos (Meila, 2005; Antzoulatos \& Vrahatis, 2011). 



\section{Caracterização de Variação de}

\section{Comportamento em Fluxos de Dados}

\subsection{Considerações iniciais}

Pesquisas atuais em agrupamento de fluxos de dados têm se concentrado em prover algoritmos cuja execução apresente baixa complexidade de tempo e espaço (Xu \& Wunsch, 2005). Essas pesquisas assumem a observação de fenômenos razoavelmente estáveis, a partir dos quais, caso o processo indutivo inicial seja bem projetado, agrupamentos serão incrementalmente construídos e a qualidade de suas hipóteses aumentarão em função da coleta de mais dados, ou seja, mais evidências que confirmam, refinam ou refutam as hipóteses.

A premissa de estabilidade não é adequada para o agrupamento de diversos fluxos de dados, durante o qual é possível que ocorram variações comportamentais do fenômeno em estudo. No início do processo de agrupamento, assim como em outros processos indutivos, essas características são estudadas para definir as premissas de funcionamento para o projeto de algoritmos, ou seja, o viés indutivo (Kifer et al., 2004; Pavlidis et al., 2011).

A variação de comportamento de fluxos de dados é a característica mais desafiadora para o projeto desses algoritmos pois prejudica o bom funcionamento da indução ao longo 
da coleta de dados. Em Aprendizado de Máquina, a tarefa de identificar variações comportamentais é conhecida como detecção de novidades (Markou \& Singh, 2003). Dessa maneira, técnicas de detecção de novidades podem ser utilizadas para avaliar e adaptar o viés indutivo de algoritmos de agrupamento em função de comportamentos presentes nos dados.

A seguir, este capítulo apresenta conceitos e técnicas de caracterização de comportamento e detecção de novidades, de acordo com o objetivo estabelecido para esta tese. Observa-se a utilização desses conceitos em diversas áreas, tais como Psicologia (Kaufmann, 2004), Biologia (Middleton, 1996), Medicina (Bardo et al., 1996) e Economia (Bertarelli \& Censolo, 2000). Esses conceitos e técnicas foram organizados, conforme requisitos de aplicações, em uma hierarquia apresentada por Albertini \& Mello (2008).

\subsection{Conceitos e técnicas}

Em Biologia, o conceito de novidade é associado aos mecanismos de sobrevivência de animais e reconhecimento de perigos (Middleton, 1996). Em Economia, novidade tem sido relacionada com a determinação de preços de bens de consumo e o surgimento de nichos de mercado especializados (Bertarelli \& Censolo, 2000). Em Psicologia, determina-se a magnitude de novidades para a caracterização de comportamentos criativos (Kaufmann, 2004). De modo geral, essa diversidade de conceitos relacionados a novidades é resultado da necessidade de reconhecer e adaptar-se a mudanças.

Em Computação e Estatística, essa necessidade é demonstrada por meio do desenvolvimento de técnicas de Aprendizado de Máquina que visam se adaptar a variações comportamentais observadas em fenômenos. Nesse contexto, novidades referem-se à identificação de informações inesperadas, raras ou desconhecidas segundo um modelo referência de conhecimento.

Técnicas de detecção de novidades em Aprendizado de Máquina são utilizadas em um amplo espectro de aplicações, tais como diagnóstico de doenças (Middleton, 1996), identificação de variacões de tendências climáticas (Lau \& Weng., 1995: Hasselmann., 1997; Luterbacher et al., 2004) e detecção de falhas em discos rígidos (Hughes et al., 2002). Essas aplicações apresentam particularidades que regem o desenvolvimento de técnicas especializadas. Dentre tais particularidades, estão a necessidade por aprendizado contínuo, esquecimento ou predição. A heterogeneidade de aplicações originou várias nomenclaturas para o termo novidade, tais como: anomalia, falha, desvio abrupto, ruído, outlier e exceção (Hodge \& Austin, 2004).

Essa variabilidade de áreas e termos motiva pesquisadores a realizar levantamentos de trabalhos relacionados visando o mapeamento, comparação de técnicas e identificação das atuais tendências de desenvolvimento. Por meio desses levantamentos, áreas aparentemente distintas podem ser relacionadas, contribuindo para o intercâmbio de técnicas e conceitos. 


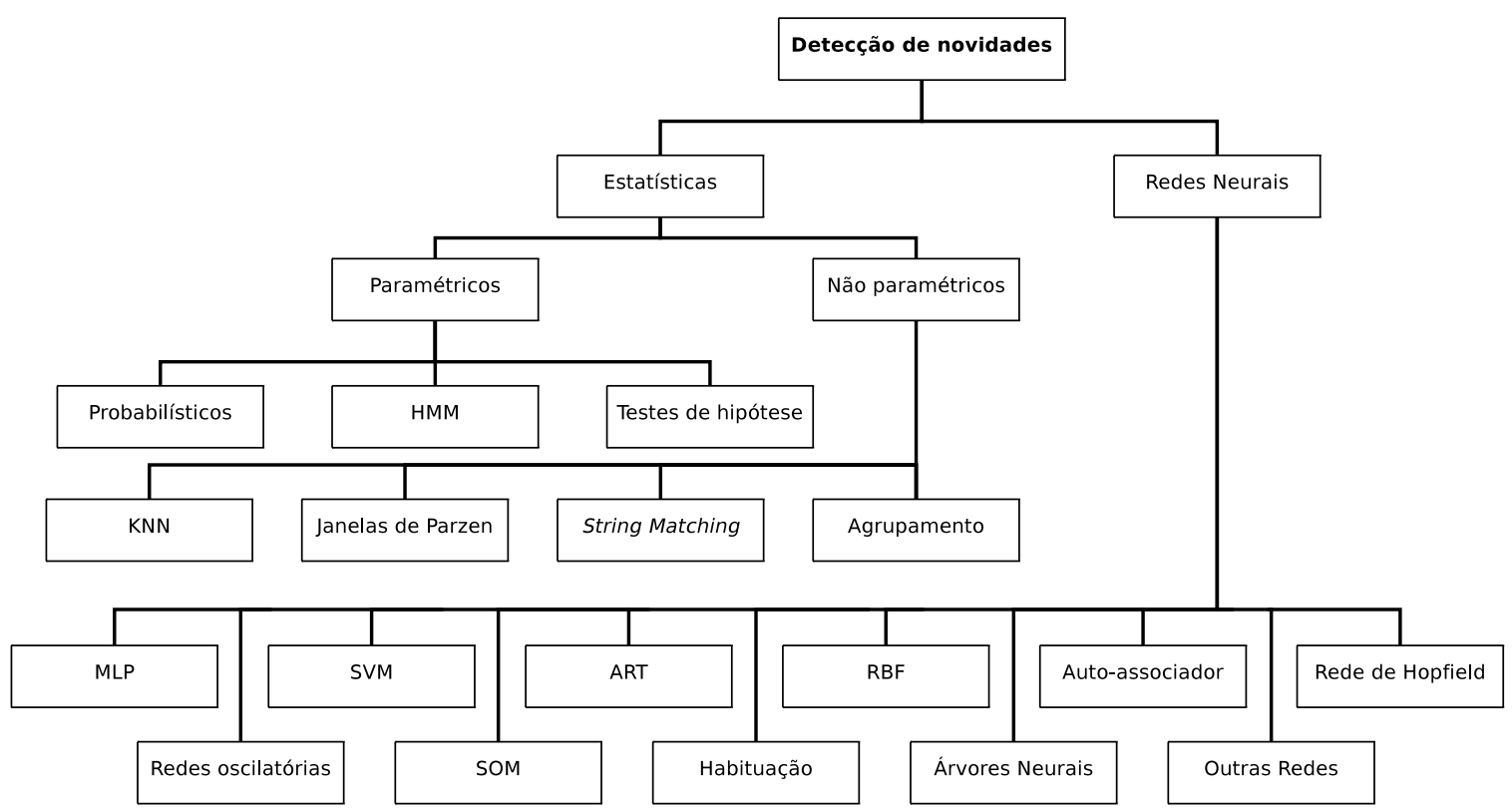

Figura 3.1: Árvore de classificação de técnicas proposta por Singh \& Markou (2005)

Um dos primeiros levantamentos realizados sobre detecção de novidades foi o trabalho de Willsky (1976), focado na área de detecção de falhas em sistemas dinâmicos lineares. Para Willsky, a detecção de falhas é composta por três tarefas: alarme, isolamento e estimação. Nesse contexto, a tarefa de alarme consiste em saber se houve alguma falha. O problema de isolamento consiste em determinar o tipo e a origem da falha. A estimação envolve identificar a extensão do problema no sistema (se a fonte de falha não está operante ou está sob algum tipo de dano). A tarefa de alarme de Willsky é uma atividade de detecção de novidades. Essa tarefa é realizada por meio da verificação de consistência de estimativas de parâmetros em modelos do sistema.

Enquanto Willsky dedicou-se a questões específicas de detecção em sistemas dinâmicos lineares, outros levantamentos mais recentes, como o realizado por Markou \& Singh (2003), envolvem uma gama mais ampla de aplicações e técnicas. Nesse levantamento, técnicas são classificadas, conforme apresentado na Figura 3.1, segundo os paradigmas baseados em redes neurais artificiais e abordagens estatísticas. Entre as redes neurais encontram-se Multi-Layer Perceptron (MLP), Support-Vector Machine ( $(\underline{\mathrm{SVM}})$, Adaptive Resonance Theory (ART), Radial Basis Function Network (RBF), rede neural artificial de Hopfield, Self-Organizing Map (SOM), auto-associadores (e.g., MLP, Principal Component Analysis, SoM), baseada em habituação (e.g., Grow When Required - GWR), Árvores Neurais (Neural Tree) e redes oscilatórias. As técnicas estatísticas incluem métodos probabilísticos, testes de hipóteses, modelos de Markov com estados escondidos (Hidden Markov Models - HMM), K-Nearest Neighbors ( $\mathrm{KNN}$, janelas de Parzen, abordagens de agrupamento e de casamento de cadeias (do inglês, string matching), tais como as utilizadas em sistemas imunológicos artificiais.

A divisão realizada por Markou \& Singh (2003) identifica os tipos de técnicas utili- 
zadas sem analisar as características de construcão do modelo de conhecimento. Hodge \& Austin (2004), sob a nomenclatura de detecção de outliers, identificaram três abordagens com diferentes paradigmas de aprendizado aplicadas na construção de modelos de conhecimento para detecção de novidades. A primeira é baseada em aprendizado não supervisionado e utiliza dados normais e novidades sem prévia rotulação. A segunda utiliza uma abordagem supervisionada baseada na classificação de dados previamente conhecidos e rotulados. A terceira é realizada por meio de aprendizado semisupervisionado, utilizando apenas informações identificadas como normais.

Os trabalhos de Markou \& Singh (2003) e Hodge \& Austin (2004) avaliaram e classificaram técnicas de detecção de novidades sob o enfoque das abordagens utilizadas. Entretanto, a linha de desenvolvimento de tais técnicas advém dos requisitos impostos pelas aplicações. Atualmente, um dos principais requisitos é resultante das aplicações que necessitam detectar novidades em sequências de dados (Al-Subaie \& Zulkernine, 2006). Tais requisitos distinguem-se pela modelagem explícita e detecção de variações de relações de causa e efeito que são denominadas nesta tese de novidades temporais.

Considerando a distinção entre as abordagens propostas para novidades temporais e as somente voltadas para aplicações não temporais, i.e., com novidades não temporais, propõe-se, em função dos trabalhos estudados, a hierarquia de classificação apresentada na Figura 3.2. Nessa hierarquia, abordagens que consideram novidades não temporais, representadas pelo Ramo 2a, são aquelas que apresentam definições de novidades sem representação explícita de causa e efeito entre dados. Elas são divididas em dois subgrupos segundo os conceitos aplicados: as abordagens estatísticas (Ramo 3a), e métodos de agrupamento e classificação (Ramo 3b).

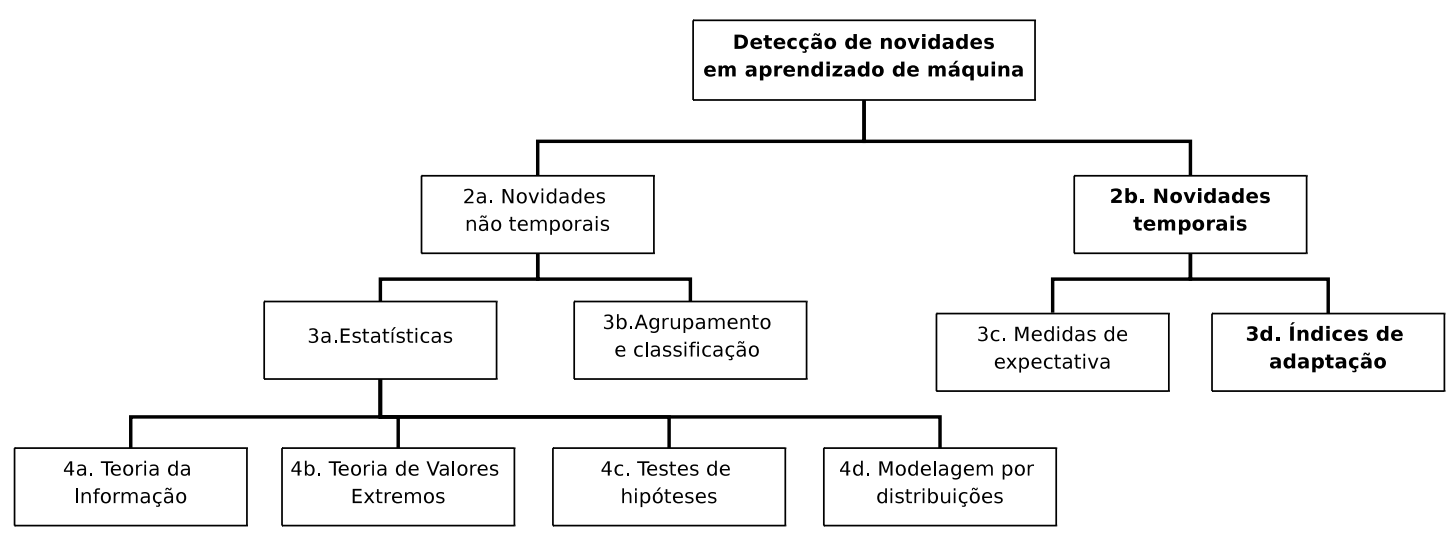

Figura 3.2: Árvore hierárquica de detecção de novidades em Aprendizado de Máquina

As abordagens do subgrupo Estatísticas são divididas na forma: aplicações de conceitos de Teoria da Informação (Ramo 4a), teoremas de Teoria de Valores Extremos (Ramo 4b), técnicas baseadas em testes de hipóteses de inferência estatística (Ramo 4c) e modelagem por meio de distribuições de probabilidades (Ramo 4d). O Ramo 4a, i.e., Teoria da Informação, refere-se a conjuntos de conceitos e teoremas voltados para analisar a geração 
de informações. Shannon (1948) definiu entropia como a quantidade média de informação de uma fonte aleatória de dados e self-information como a medida de informação de cada possível evento gerado por essa fonte. O conceito de self-information foi considerado por Tribus (1961) para quantificar níveis de surpresas. Uma aplicação dos conceitos de entropia foi proposta por Itti \& Baldi (2005), que avaliaram e compararam a entropia de quadros consecutivos de vídeos à surpresa que tais imagens geravam em humanos, visando estabelecer uma relação entre surpresa e entropia. Nesse trabalho, identificou-se que as áreas mais observadas por humanos coincidem com aquelas de maior entropia.

O Ramo 4b, i.e., Teoria de Valores Extremos (Roberts, 1999), contém abordagens baseadas em teoremas para a representação de distribuições assintóticas de funções de probabilidades, ou seja, das regiões extremas em que novidades são geralmente originadas. De acordo com o teorema de Fisher \& Tippett (1928), funções que respeitem condições relativamente genéricas têm distribuições assintóticas de apenas três tipos: de Gumbel, de Fréchet ou de Weibull. Roberts (1999) utiliza esse teorema para aplicações com regiões extremas na forma da distribuição de Gumbel. Ele aplica um método de aproximação por meio de mistura de gaussianas sobre dois conjuntos de dados: o primeiro contendo informações sobre epilepsia e, o segundo, ruídos em imagens. Bensalah (2000) utiliza conceitos de Teoria de Valores Extremos para obter informações sobre riscos em aplicações financeiras.

O Ramo 4c, i.e., testes de hipóteses de inferência estatística, considera conceitos voltados para avaliar a consistência de estimação de parâmetros de modelos. Exemplos de técnicas que utilizam tal abordagem são encontrados no levantamento bibliográfico de Willsky (1976) que cita a utilização de testes de hipótese Qui-quadrado para verificar a consistência de parâmetros estimados em modelos de sistemas eletrônicos. Outro exemplo de aplicação é encontrado em Hughes et al. (2002), o qual utiliza o teste de hipótese Wilcoxon Rank-Sum (Wilcoxon, 1945; Mann \& Whitney, 1947) com o objetivo de reduzir a taxa de alarmes falsos de um sistema de diagnóstico e predição de falhas em discos rígidos, denominado Self-Monitoring, Analysis and Reporting Technology (SMART). O teste utilizado avalia se dois conjuntos amostrais foram obtidos a partir da mesma função de distribuição de probabilidades. Outros métodos baseados em testes de hipóteses podem ser encontrados no livro de tratamento estatístico de outliers de Barnett \& Lewis (1994).

O Ramo 4d, i.e., modelagem por meio de distribuições de probabilidades, refere-se a técnicas para construção de modelos de representação de informações por meio de funções de distribuição de probabilidades. Um exemplo de abordagem desse tipo foi proposto por Bishop (1995), o qual detecta novidades estimando a densidade de dados de entrada e rotulando informações identificadas em áreas de baixa probabilidade. A mesma abordagem foi aplicada por Tarassenko et al. (1999) com o objetivo de detectar regiões cancerígenas em mamogramas e por Lauer (2001), a partir de um método similar, sobre um conjunto de dados de diagnóstico médico, denominado BIOMED, com o objetivo de identificar portadores de uma rara desordem 
genética.

O segundo subgrupo do ramo de técnicas sem novidades temporais reúne métodos baseados em agrupamento e classificação (Ramo 3b). Esses métodos apresentam duas abordagens distintas e podem atuar por meio da classe de identificação ou pela aplicação de medidas de avaliação.

As abordagens que utilizam a classe de identificação utilizam supervisão para definir a diferença entre elementos normais e novidades. Um exemplo é o trabalho de Rosen et al. (1996), que adota uma rede neural artificial multi-layer perceptron para identificar tendências anormais em batimentos cardíacos de fetos (cardiotocografia). De maneira similar. Spinosa \& de Carvalho (2005) utilizam a técnica de classificação binária Support Vector Machine ( $(\mathbf{S V M})$, baseada na Teoria de Aprendizado Estatístico de Vapnik (1995), para detectar novidades nos conjuntos de dados de bioinformática ALL-AmL Leukemia (Golub et al., 1999) e Diffuse Large B-cell Lymphoma (DLBLC) (Alizadeh et al., 2000). Adotando os mesmos conceitos, Flexer et al. (2005) aplicam detecção de novidades para recuperar músicas por meio da similaridade de espectro e rotulação de estilos. Nesse trabalho, dois minutos centrais de cada música são avaliados e novidades são detectadas utilizando dois algoritmos. O primeiro, denominado Ratio-reject, utiliza uma distribuição de densidade de dados, obtida na fase de treinamento, para classificação. O segundo algoritmo, denominado KNN-reject, define uma vizinhança durante o treinamento, a qual é utilizada para classificar dados conhecidos ou novidades.

Por outro lado, abordagens do Ramo 3b, que utilizam medidas de avaliação, consideram a instabilidade de algoritmos de classificação para detectar novidades. Com esse objetivo, Ypma \& Duin (1997) utilizam a rede auto-organizável Self-Organizing Maps de Kohonen (1997) adotando diferentes parâmetros de aprendizado. Avalia-se cada mapa criado por meio de métricas de qualidade de representação como, por exemplo, distância dos dois neurônios com maior ativação no mapa. Experimentos indicam que conjuntos de dados com anomalias geram valores de erro de classificação maiores que conjuntos normais. Em uma abordagem similar, Tax \& Duin (1998) propõem a utilização de um método de amostragem aleatória, com reposição, em que são criados novos conjuntos do mesmo tamanho do conjunto de dados original. Cada novo conjunto é classificado e as diferenças nos resultados de classificação são utilizadas para definir anormalidades. Segundo os autores, uma novidade causa instabilidade nos resultados de classificação.

No lado direito da hierarquia da Figura 3.2 estão técnicas baseadas em novidades temporais (Ramo 2b), que modelam explicitamente o comportamento de dados para a detecção de variações em relações de causa e efeito entre dados. Técnicas que detectam esse tipo de novidade são classificadas em: medidas de expectativa (Ramo 3c) e índices de adaptação (Ramo 3d).

As técnicas sob o Ramo 3c, i.e., medidas de expectativa, definem novidades como a 
distância de um valor obtido em relação a uma medida de expectativa. Medidas frequentemente utilizadas são valores esperados e predições. As técnicas sob o Ramo 3d, i.e., índices de adaptação, avaliam o quanto um modelo de conhecimento é modificado após a inserção de dados.

Um exemplo de trabalho sob o Ramo 3c, i.e., medidas de expectativa, é o proposto por Marsland (2002). Nesse trabalho foi projetada uma rede neural artificial, denominada Grow When Required, em que neurônios são capazes de avaliar a frequência de padrões por meio de uma métrica inspirada em um fenômeno biológico denominado habituação. Nessa métrica, quanto maior a habituação de um neurônio, menor é a novidade de um padrão a ele relacionado. Em outro exemplo, Ma \& Perkins (2003) propuseram uma técnica em que novidades são definidas na forma de sequências de dados com um número mínimo de surpresas, as quais ultrapassam um valor de expectativa máximo. A modelagem é realizada utilizando uma função de regressão com vetores de suporte, a qual permite atualização incremental. Essa função é utilizada para garantir graus de confiança a novidades, considerando o número consecutivo de surpresas.

Técnicas para detecção de novidades temporais a partir do uso de índices de adaptação, apresentam desenvolvimento recente. Dentre os trabalhos classificados sob esse ramo, Priebe et al. (2005) analisaram um conjunto de dados contendo informações sobre a troca de mensagens de correio eletrônico da empresa de energia norte-americana Enron, durante uma época em que ocorreram fraudes contábeis. Essa análise foi conduzida com o objetivo de encontrar anomalias na comunicação interna da empresa por meio de estatísticas de variação de grafos. Por meio dessas estatísticas buscou-se regiões de grafos com excessiva comunicação, as quais caracterizavam anomalias. Para detectar novidades em vídeos, Itti \& Baldi (2005) propõem o uso de entropia relativa, ou divergência de Kullback-Leibler (Kullback, 1959), para medir a diferença de modelos probabilísticos do conteúdo de imagens em instantes de tempo consecutivos. Ainda sob o mesmo ramo, Gamon (2006) aborda a detecção de novidades em textos jornalísticos considerando contexto prévio. Com esse objetivo, modela-se sentenças dos documentos em grafos. Após a inserção de novas sentenças, medidas de modificação do modelo (e.g., número de vértices e arestas, grau de conectividade médio e soma de pesos das arestas) são consideradas com objetivo de determinar o grau de variação do modelo de conhecimento e, portanto, detectar novidades em coleções de textos.

\subsection{Considerações finais}

A variação de comportamento de fenômenos é, atualmente, o fator mais desafiador para o projeto de algoritmos de agrupamento de fluxos de dados. A detecção de novidades é essencial, portanto, para a avaliação do impacto de variações comportamentais de fenômenos 
na adaptação do viés indutivo de algoritmos.

Considerando a distinção entre as diversas abordagens existentes para a detecção de novidades, que se diferenciam no contexto desta tese entre novidades temporais, capazes de representar variações comportamentais em sequências de dados, e as voltadas para aplicações não temporais, i.e., com novidades não temporais, propôs-se neste trabalho a hierarquia de classificação anteriormente apresentada.

A partir dessa hierarquia de classificação, observa-se, no contexto de fluxos de dados, a importância da utilização de medidas de novidades temporais a partir de técnicas que modelam explicitamente o comportamento de fenômenos para cumprir o objetivo desta tese, que é adaptar o viés indutivo de algoritmos de agrupamento de fluxos de dados. 


\section{4}

\section{Adaptação de Viés Indutivo de}

\section{Algoritmos de Agrupamento de Fluxos}

de Dados

\subsection{Considerações iniciais}

Diversas áreas de pesquisa são dedicadas à compreensão de fenômenos que exigem a coleta ininterrupta de sequências de amostras, denominadas fluxos de dados. Esses fenômenos frequentemente apresentam comportamento variável e são estudados por meio de indução não supervisionada baseada em agrupamento de dados.

Atualmente, o processo de agrupamento tem exibido sérias limitações em sua aplicação a fluxos de dados, devido às exigências impostas pelas variações comportamentais e pelo modo de coleta de dados.

Embora pesquisadores têm se dedicado ao desenvolvimento de algoritmos com baixa complexidade espaço-temporal para processar grandes volumes de dados obtidos a altas taxas, há a necessidade de estudos sobre a interferência de variações comportamentais nos valores adequados para os parâmetros de algoritmos (e.g., taxas de aprendizado e limiares de proximi- 
dade), que guiam a produção de grupos, influenciando na compreensão efetiva do fenômeno em estudo.

Portanto, observou-se lacunas no que se refere à adaptação do viés indutivo de algoritmos de acordo com variações comportamentais. Estabeleceu-se, dessa forma, como objetivo desta tese o desenvolvimento de uma abordagem de adaptação do viés indutivo de parâmetros de algoritmos de agrupamento de acordo com medidas de caracterização de variações de comportamento em fluxos de dados. A seguir, são apresentados estudos e atividades para atingir tal objetivo.

\subsection{Histórico de desenvolvimento da tese}

Esta seção fornece, em linhas gerais, uma descrição do histórico de desenvolvimento desta tese de doutorado com o objetivo de oferecer ao leitor uma perspectiva das decisões de projeto que ocorreram ao longo de sua elaboração e um resumo das atividades realizadas.

Em 2005, estudos de iniciação científica realizados pelo autor desta tese sobre detecção de faltas em Sistemas Computacionais Distribuídos (Albertini \& Mello, 2005) evidenciaram a importância de compreender fenômenos complexos a partir da modelagem automática de dados. Nesse contexto, modelos são utilizados para distinguir o comportamento normal das variações de comportamento apresentadas em um sistema com faltas. Observou-se, também, que, em fenômenos complexos, dados são frequentemente coletados a altas taxas e quantidades, o que motivou a condução de estudos sobre técnicas de modelagem de fluxos de dados. Esses estudos evidenciaram a importância de considerar as influências de variações comportamentais presentes em fluxos de dados durante o processo de modelagem, uma vez que elas representam informações importantes e podem interferir na compreensão de fenômenos (Mello et al., 2010; Itti \& Baldi, 2005; Gamon, 2006).

$\mathrm{Na}$ área de Aprendizado de Máquina, a tarefa de identificar variações comportamentais de fenômenos é denominada detecção de novidades (Markou \& Singh, 2003). O aprofundamento nesse tema permitiu o conhecimento das diversas abordagens utilizadas sob pontos de vista de diferentes domínios, conforme apresentado nos Capítulos $2 \mathrm{e} 3$.

A partir do conhecimento acumulado nesses estudos, pôde-se observar que atualmente grandes esforços têm sido dispendidos na busca por técnicas para, uma vez mais, transformar a maneira de compreender fenômenos complexos, a partir da coleta de quantidades de dados cada vez maiores (Hey et al., 2009). Esses esforços têm sido guiados pela necessidade de evidenciar tendências, obter informações e descrever princípios de fenômenos que, devido à quantidade de dados necessária para sua descrição ou a sua alta complexidade, especialistas não são capazes de efetivamente analisá-los, estudá-los e, portanto, compreendê-los com as ferramentas atualmente disponíveis. 
Dentre as principais alternativas consideradas para compreender tais fenômenos, que não apresentam informações providas por agentes externos ou especialistas, destacam-se métodos não supervisionados de indução, tais como algoritmos de agrupamento de dados, redes neurais artificiais e modelos estatísticos (Markou \& Singh, 2003). O estudo de técnicas utilizadas em agrupamento de dados, tais como Self-Organizing Maps (Kohonen, 1997), Growing K-médias (Daszykowski et al., 2002), Sequential Leader Clustering (Hartigan, 1975) e Grow When Required (Marsland, 2002), permitiu observar que esses algoritmos utilizam limiares de pertinência de dados a grupos na forma de parâmetros, os quais são definidos na etapa de projeto do algoritmo de agrupamento (Figura 1.1), a partir da análise de amostras inicialmente coletadas. Nessa etapa, especialistas buscam definir valores para esses limiares com o intuito de obter a melhor partição possível para todo o conjunto de dados.

Contudo, observou-se que variações de comportamento presentes em fluxos de dados interferem na execução de algoritmos para a obtenção de bons modelos e que os limiares estabelecidos na etapa de projeto podem se tornar inadequados ao longo do tempo. Essa observação motivou a proposta de uma arquitetura de rede neural artificial denominada Self-Organizing Novelty Detector (SONDE) (descrita na Seção 4.4), a qual é baseada em equações de aprendizado para a contínua obtenção de estruturas de conhecimento a fim de caracterizar o comportamento do fenômeno em estudo. Devido a avaliações experimentais de algoritmos de agrupamento e de detecção de novidades, optou-se por definir que o aprendizado da arquitetura SONDE seria baseado em equações de médias móveis exponenciais ponderadas, conforme apresentado na Seção 4.4. Essas equações de aprendizado são descritas por meio de parâmetros de ponderação, constituintes do viés indutivo dessa arquitetura, que conduzem à atualização de estruturas de conhecimento baseadas em protótipos de grupos e da dispersão de padrões em torno desses protótipos. Por sua vez, o limiar de pertinência de dados a grupos seria, diferentemente das técnicas então avaliadas, adaptado continuamente, de acordo com as estruturas de conhecimento.

Imediatamente se notou que os parâmetros das equações de aprendizado da SoNDE também necessitariam de adaptação de acordo com as variações comportamentais observadas em fluxos de dados. De forma geral, conjecturou-se que qualquer parâmetro constituinte do viés indutivo de agrupamento, cujo valor inicial é definido na etapa de projeto, necessitaria de adaptação ao longo do processamento de fluxos de dados. A partir dessa conjectura surgiram questionamentos sobre como esses parâmetros deveriam ser adaptados.

Portanto, a necessidade de compreender fenômenos com variação de comportamento a partir da coleta de fluxos de dados e a falta de técnicas que continuamente adaptam parâmetros de agrupamento motivaram a definição da hipótese desta tese de doutorado:

Hipótese: Adaptando o viés indutivo de algoritmos de agrupamento de fluxos de 
dados de acordo com variações comportamentais do fenômeno em estudo pode-se garantir a contínua obtenção de conhecimento.

A partir dos estudos e experimentos realizados e da hipótese estabelecida, definiu-se como objetivo desta tese:

Objetivo: Projetar e desenvolver uma abordagem de adaptação do viés indutivo de parâmetros de algoritmos de agrupamento de fluxos de dados, considerando fenômenos que apresentam variação comportamental.

A abordagem idealizada nesse objetivo foi estabelecida, conforme o diagrama ilustrado na Figura 1.2, para conter três principais componentes: i) um algoritmo de agrupamento de fluxos de dados; ii) uma medida para avaliar variações comportamentais presentes em fluxos de dados; iii) e uma técnica de adaptação de viés indutivo por meio de parâmetros do algoritmo de agrupamento de acordo com a variação de comportamento de fenômenos.

(i)

Algoritmo de
Agrupamento $\longrightarrow \begin{gathered}\text { Avaliação de } \\ \text { Comportamento }\end{gathered} \longrightarrow \begin{gathered}\text { Adaptação de } \\ \text { Viés Indutivo }\end{gathered}$

Figura 1.2: Diagrama de componentes da abordagem idealizada no objetivo desta tese

Ao longo da elaboração desta tese, encontrou-se diferentes opções de projeto para a abordagem idealizada, de acordo o objetivo estabelecido. Dentre essas opções, concentraram-se os esforços de execução na proposta de três abordagens que auxiliaram na comprovação da hipótese desta tese.

Os estudos realizados para a primeira abordagem basearam-se, conforme os componentes apresentados na Figura 1.2, em: i) utilizar a arquitetura de rede neural artificial auto-organizável Self-Organizing Novelty Detector (SONDE) no agrupamento de dados de maneira adaptativa e incremental; ii) avaliação de comportamento presente em fluxos de dados por meio da estimação de cadeias de Markov (Seneta, 2006) e da entropia de Shannon (1948); e iii) projeto de uma técnica de adaptação de viés indutivo por meio da minimização da variação de entropia de cadeias de Markov ocorrida em virtude da inadequação dos parâmetros que constituem o viés da arquitetura SONDE,

Nessa primeira abordagem, descrita na Seção 4.6, a arquitetura projetada SONDE (Seção 4.5) permite representar grupos por meio de equações de aprendizado baseadas em proximidade espacial entre elementos, as quais possuem parâmetros que constituem seu viés 
indutivo. A representação de grupos por meio de neurônios da SoNDE permite a estimação da matriz de probabilidades de transições de cadeias de Markov. A avaliação comportamental do fenômeno em estudo é baseada na variação de entropia entre cadeias de Markov consecutivas (Mello et al., 2010; de Gouveia et al., 2011).

Essa medida foi utilizada no projeto da técnica de adaptação de viés indutivo inspirada em um método de otimização estocástica de gradiente descendente. O projeto de tal técnica partiu da hipótese que a variação de entropia entre cadeias de Markov consecutivas é um produto de modificações de agrupamentos presentes em fluxos de dados e da inadequação do viés indutivo em atender variações de comportamento do fenômeno em estudo. Dessa forma, o objetivo dessa técnica foi adaptar os parâmetros de aprendizado da SoNDE a fim de corrigir as inadequações apresentadas em seu viés indutivo. Para isso, buscou-se projetar tal técnica na forma de um método de otimização baseado em gradiente descendente, similar ao utilizado em redes neurais artificiais com treinamento baseado em retropropagação de erros (Mitchell, 1997). Um método de gradiente descendente visa otimizar uma função de custo, conhecida como função-objetivo, por meio de uma busca orientada pela direção de maior variação da função, ou seja, o seu gradiente.

Contudo, apesar de exaustivos esforços em seu projeto e desenvolvimento, a primeira abordagem provou-se de difícil concretização graças a problemas na obtenção da funçãoobjetivo da técnica de adaptação de viés indutivo. Tradicionalmente, métodos baseados em gradiente descendente exigem a descrição dessa função a partir do conhecimento analítico sobre a superfície a ser explorada. No caso da primeira abordagem, a superfície da funçãoobjetivo da técnica de adaptação é formada por elementos desconhecidos ou variantes, tal como a distribuição de probabilidades do fluxo de dados. Para contornar essa limitação, foram propostas aproximações e hipóteses simplificadoras para o comportamento assintótico de aprendizado de fluxos de dados. Porém, apesar de plausíveis, essas aproximações provaram-se ineficazes e os resultados obtidos em experimentos foram insatisfatórios.

A ineficácia da primeira abordagem, originada nas dificuldades em caracterizar a relação entre o viés indutivo e variações comportamentais presentes em fluxos de dados, motivou o desenvolvimento de uma segunda proposta, denominada Data Stream Dynamic Clustering (DSDC) DSDC (Albertini \& Mello, 2013).

Essa abordagem constituiu, conforme os componentes na Figura 1.2, da: i) utilização do algoritmo de agrupamento tradicional de dados K-médias (Hartigan, 1975) para a produção de partições em blocos de subsequências; ii) estimação de propriedades assintóticas de cadeias de Markov para a verificação de equivalência de comportamento entre partições consecutivas (Ornstein, 1989; Seneta, 2006); e iii) aplicação de um algoritmo de otimização que define o número de grupos para o algoritmo K-médias de acordo com a medida de qualidade de partições Silhueta (Rousseeuw, 1987). 
Nessa segunda abordagem, a detecção de variações de comportamentos do fenômeno utiliza a verificação de isomorfismo do comportamento assintótico entre cadeias de Markov estimadas a partir de partições, as quais são obtidas com o algoritmo K-médias aplicado em blocos de subsequências de dados (Ornstein, 1989). Para adaptar os parâmetros de agrupamento disponíveis para o algoritmo K-médias, ou seja, o número de grupos $k$, utilizou-se uma busca exaustiva pelo valor de $k$, o que provê um agrupamento com melhor qualidade, conforme a medida Silhueta (Rousseeuw, 1987).

A proposta da abordagem DSDC, apesar de não permitir a adaptação do viés indutivo de maneira contínua, segundo estabelecido na hipótese desta tese, permitiu confirmar a melhora de qualidade da sequência de partições obtida quando o parâmetro do algoritmo de agrupamento é atualizado, confirmando a possibilidade de melhor representar fenômenos com comportamento variável.

Além disso, essa proposta foi útil para verificar que algoritmos baseados em divisão de fluxos de dados em blocos é limitada, pois é difícil determinar o tamanho ótimo para esses blocos. Essa limitação também prejudica a obtenção de partições e a estimativa de cadeias de Markov, o que resulta em falsos positivos para a detecção de variação de comportamento e, portanto, na realização de buscas desnecessárias pelo parâmetro $k$. Esse fato é atribuído ao uso de blocos de dados em vez de atualização contínua de agrupamentos. Além disso, deve-se ter em conta que o emprego do algoritmo K-médias com inicialização de protótipos aleatórios não garante a continuidade das partições, o que também pode interferir na detecção de variação de comportamento.

Contudo, conforme descrito na hipótese desta tese, ainda se observou a necessidade de uma abordagem contínua para realizar adaptações ao viés indutivo ao longo da obtenção dos dados, provendo informações imediatas relativas às variações de comportamento de fenômenos. Dessa forma, motivou-se a realização de estudos para o projeto de uma terceira abordagem, considerando a adaptação contínua do viés indutivo do algoritmo de agrupamento de fluxo de dados.

Esses estudos basearam-se, conforme os componentes na Figura 1.2, em: i) utilização da arquitetura SONDE como algoritmo de agrupamento; ii) emprego de uma medida de estabilidade do comportamento presente no fluxo de dados; e iii) projeto de uma técnica de adaptação de viés indutivo baseada na relação entre a medida de estabilidade e os parâmetros das equações da arquitetura SONDE.

A proposta da terceira abordagem partiu da associação dos parâmetros das equações de aprendizado da SONDE à necessidade de representar a dependência temporal entre dados de acordo com a estabilidade de comportamento observada nos dados. Para isso, projetou-se uma técnica de adaptação baseada em uma função de mapeamento entre a medida de estabilidade de dados e os parâmetros das equações da arquitetura SONDE. Essa técnica atua sobre os parâ- 
metros de forma a aumentar a memória das equações de aprendizado, caso dados apresentem maior estabilidade e diminuir, caso contrário. Resultados obtidos a partir de experimentos realizados com fluxos de dados sintéticos, utilizando essa técnica, permitiram verificar as vantagens da adaptação contínua do viés indutivo de agrupamento, comprovando a hipótese desta tese.

É importante ter em conta que a dedicação de esforços do autor desta tese se concentraram no projeto dessas três abordagens brevemente apresentadas e que os resultados obtidos permitiram ampliar a compreensão do problema de agrupamento de fluxos de dados observados a partir de fenômenos com variação de comportamento.

Ainda assim, outras alternativas foram consideradas durante os estudos realizados para a proposta da abordagem pretendida. Dentre essas alternativas pode-se mencionar técnicas de adaptação baseadas em modelos descritos por equações diferenciais, tais como sistemas de amortecimento, o oscilador de van der Pol (1927) e equações obtidas por meio da transformação de Liénard (1928). Além disso, foram consideradas funções de distribuição de probabilidades, tais como a função Gama (Andrews et al., 2001), para representar a distância de dados aos protótipos em seus grupos de maneira genérica, e exaustivos estudos para a obtenção de propriedades de convergência de aprendizado para a arquitetura SONDE. Porém, essas alternativas foram, por diferentes motivos, consideradas inviáveis para a realização de estudos aprofundados.

Adicionalmente, estudos de formalização do problema de agrupamento de conjuntos tradicionais de dados (Kleinberg, 2002) permitiram estabelecer, nesta tese, uma formalização similar para agrupamento de fluxos de dados. Essa formalização auxiliou na compreensão das propriedades únicas ao cenário de agrupamento de fluxos e na caracterização da natureza do viés indutivo de algoritmos de agrupamento de fluxos de dados.

Nas seções a seguir, são apresentados maiores detalhes sobre os estudos anteriormente mencionados. Primeiramente, na Seção4.3, são introduzidos conceitos sobre a constituição do viés de indutivo de algoritmos de agrupamento. A seguir, apresenta-se a arquitetura SONDE, Após isso, é descrito um método de estimação de cadeias de Markov com base em agrupamento de fluxos de dados e caracterização de variação de comportamento de fenômenos a partir da medição da entropia de Shannon (Seção 4.5). Esse método é utilizado no equacionamento da primeira abordagem de adaptação de viés indutivo de agrupamento (Seção 4.6). Na seção seguinte, descreve-se a segunda abordagem desta tese, o algoritmo Data Stream Dynamic Clustering (DSDC). Após isso, na Seção 4.8, a terceira abordagem de adaptação do viés indutivo, baseada na avaliação da estabilidade de fluxos de dados, é apresentada. Enfim, são feitas considerações finais. 


\subsection{Vieses de algoritmos de agrupamento}

Formalmente, modelos são conjuntos de hipóteses sobre as propriedades dos dados. Um modelo é considerado ideal, segundo o princípio da Navalha de Occam ${ }^{1}$ (Hawking, 2003, p. 731), quando é descrito pelo menor conjunto de hipóteses capaz de explicar correta e completamente o fenômeno observado. Essas hipóteses são formadas por meio de elementos de uma linguagem adequadamente projetada com o objetivo de representar as possíveis tendências e propriedades dos dados, a linguagem de representação de hipóteses. O conjunto de todas as hipóteses que tal linguagem é capaz de descrever é conhecido como o espaço de representação da linguagem (Mitchell, 1997). Esse espaço é determinado pelas propriedades e condições estabelecidas em seu projeto e está contido no universo de todas as hipóteses. Tais propriedades e condições especializam o uso da linguagem e são conhecidas em Aprendizado de Máquina como viés indutivo de linguagem (Dehaspe \& Raedt, 1996).

Como exemplo, observe na Figura 4.1, uma ilustração ${ }^{2}$ que retrata os vieses para duas diferentes linguagens. O conjunto universo de todas as hipóteses é representado pelo retângulo U, que, em agrupamento, é constituído por todas as partições possíveis dos dados. Os subespaços de todas as hipóteses descritas para duas diferentes linguagens de representação são retratados pelos conjuntos $\mathcal{L}_{1}$ e $\mathcal{L}_{2}$. Note que elas compartilham similaridades e contêm hipóteses em comum.

O projeto de algoritmos indutivos deve também considerar condições ideais para sua aplicação no fenômeno observado. Essas condições, denominadas viés indutivo de preferência, ou viés de preferência, estabelecem, dentro do subespaço definido pelo viés indutivo de linguagem, um subespaço de busca de hipóteses em que o algoritmo procurará por modelos. No caso do exemplo apresentado na Figura 4.1, os subespaços de hipóteses nos quais algoritmos podem atuar, respeitando o espaço das linguagens $\mathcal{L}_{1}$ e $\mathcal{L}_{2}$, são, respectivamente, $\left\{\mathcal{A}_{1}, \mathcal{A}_{2}\right\}$ e $\left\{\mathcal{B}_{1}, \mathcal{B}_{2}\right\}$. Esses subespaços, definidos pelo viés indutivo de preferência de código, ou viés de código, são decorrentes de decisões de projeto na codificação desses algoritmos. Adicionalmente, esses subespaços definidos pelo viés de preferência de código dependem de parâmetros disponíveis na codificação dos algoritmos ${ }^{3}$, o que se denomina viés indutivo de preferência de parâmetros, ou viés de parâmetros. A partir desses parâmetros é possível guiar o viés de preferência de algoritmos. No caso do algoritmo que define $\mathcal{B}_{2}$, os subespaços $\phi_{1}$ e $\phi_{2}$ são definidos por dois diferentes conjuntos de valores para os parâmetros que orientam esse algoritmo a obter certas hipóteses em detrimento de outras.

\footnotetext{
${ }^{1}$ We are to admit no more causes of natural things than such as are both true and sufficient to explain their appearances, Isaac Newton.

${ }^{2}$ A ilustração na Figura 4.1 tem o intuito de apenas exemplificar relações de pertinência entre conjuntos e não tem o objetivo de retratar, implícita ou explicitamente, a existência de uma relação de ordem entre hipóteses (Skvarcius \& Robinson, 1986).

${ }^{3}$ Também denominados hiperparâmetros, para diferenciá-los de parâmetros de modelos estatísticos.
} 
$\mathrm{O}$ espaço definido pelos parâmetros de $\phi_{2}$ para o algoritmo $\mathcal{B}_{2}$ é aquele que mais se aproxima de um modelo ideal $\Gamma_{0}$. Na prática, a busca por um modelo ideal $\Gamma_{0}$, que seja completo, correto e parcimonioso, pode ser inviável, caso seu custo seja demasiadamente alto, ou impossível de ser atendido, caso $\Gamma_{0}$ não esteja dentro do alcance do viés indutivo determinado pelo projeto da linguagem de representação de hipóteses, do algoritmo indutivo e de seus parâmetros. Isso é, os espaços definidos pelos parâmetros de $\phi_{1}$ ou $\phi_{2}$ para o algoritmo $\mathcal{B}_{2}$, que utiliza a linguagem $\mathcal{L}_{2}$, não contêm o modelo ideal $\Gamma_{0}$. Portanto, é necessário encontrar um modelo factível, denotado $\Gamma_{1}$, que aproxima $\Gamma_{0}$ e equilibra os custos computacionais e os requisitos de ser completo, correto e parcimonioso (von Luxburg \& Schoelkopf, 2008).

Tomando como base a formalização apresentada por Kleinberg (2002), observou-se, no desenvolvimento desta tese, que os elementos que definem os vieses indutivos de linguagem, de preferência de código e de preferência de parâmetros podem ser associados ao problema de agrupamento de dados. A definição de uma função, ou matriz de similaridades, que fornece valores para a comparação entre dados é associada, pelo autor desta tese, à linguagem de representação de hipóteses, enquanto a função de particionamento, que utiliza tal matriz, ao indutor.

No caso do exemplo anterior, referente à Figura 4.1, o conjunto universo de todas as hipóteses corresponde ao conjunto de todas as possíveis partições dos elementos disponíveis, representado pelo retângulo U. Sabe-se que o espaço U é finito, porém cresce rapidamente com a quantidade de elementos a serem agrupados. Por exemplo, para um conjunto de 100 elementos, existem cerca de $4 \times 10^{115}$ diferentes configurações de agrupamento (Pitman, 1997).

Os subconjuntos de todas as partições possíveis de serem descritas para cada uma das linguagens de representação são denotados pelas elipses $\mathcal{L}_{1}$ e $\mathcal{L}_{2}$. O conjunto definido pelo viés da linguagem $\mathcal{L}_{1}$ pode representar os agrupamentos definidos por uma função de distância baseada em densidade associada ao algoritmo de agrupamento que define $\mathcal{A}_{1}$, e.g., a técnica DBSCAN (Ester et al., 1996). O conjunto $\mathcal{L}_{2}$ pode representar o uso de uma função de distância euclidiana. Nesse caso, $\mathcal{B}_{1}$ pode ser definido pelo algoritmo K-médias (Rousseeuw, 1987), que se limita a encontrar agrupamentos em subespaços com partições de somente $k$ subconjuntos, e $\mathcal{B}_{2}$, por um algoritmo de agrupamento hierárquico (Ward, 1963), que tem seu viés de parâmetros definido por um critério de corte de dendrogramas.

Os subespaços $\phi_{1}$ e $\phi_{2}$ podem representar conjuntos de agrupamentos para diferentes vieses de parâmetros estabelecidos, por exemplo, a partir de distintos critérios de corte para uma hierarquia de grupos obtida com o algoritmo Single-linkage (Ward, 1963). O subespaço $\phi_{1}$ representaria um critério de corte determinado a partir da distância mínima entre grupos e $\phi_{2}$, a partir de um critério dependente do tamanho mínimo de grupos. A partição factível $\Gamma_{1}$ é encontrada em um conjunto de partições definido em $\phi_{2}$, enquanto $\Gamma_{0}$ seria uma partição ideal, difícil ou impossível de ser encontrada, se considerado um algoritmo de agrupamento cujo viés indutivo define o subespaço $\mathcal{B}_{2}$, que não contém $\Gamma_{0}$. 


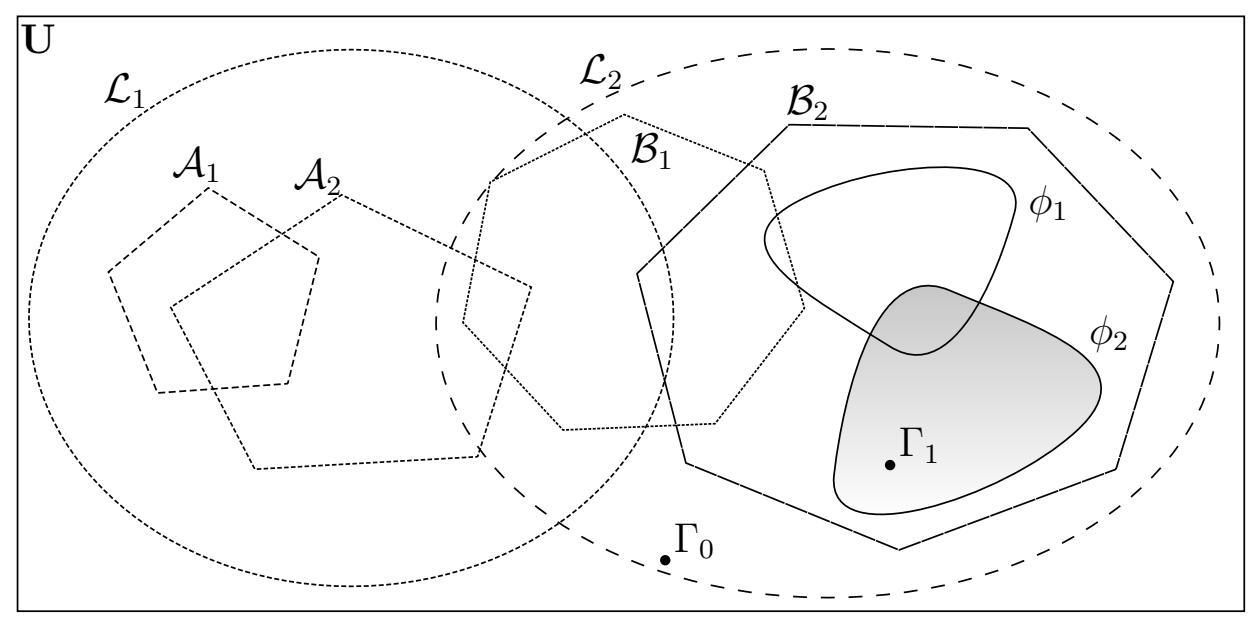

Figura 4.1: Ilustração de diferentes vieses indutivos de acordo com linguagens de representação de hipóteses $\left(\mathcal{L}_{1}\right.$ e $\left.\mathcal{L}_{2}\right)$, algoritmos indutivos $\left(\mathcal{A}_{1}, \mathcal{A}_{2}, \mathcal{B}_{1}\right.$ e $\left.\mathcal{B}_{2}\right)$ e parâmetros $\left(\phi_{1}\right.$ e $\left.\phi_{2}\right)$. $\mathrm{O}$ modelo ideal $\Gamma_{0}$ e o factível $\Gamma_{1}$ são definidos no conjunto universo de hipóteses $\mathbf{U}$

Em resumo, o sucesso do processo de agrupamento é dependente das escolhas da linguagem de representação de hipóteses, do algoritmo indutivo e de seus parâmetros. Essas escolhas são, em geral, realizadas por especialistas na etapa de projeto de algoritmos de agrupamento de fluxos de dados. Abordagens para tomar decisões relativas ao agrupamento são diversas e as mais comuns baseiam-se em: conhecimento prévio do especialista, estudos de propriedades dos dados, algoritmos de otimização com base em amostras e metadados (Brazdil, 2009).

O processo indutivo é bem sucedido desde que sejam consideradas premissas realistas que orientem a escolha de vieses adequados. Contudo, devido à natureza de fluxos de dados, ao longo desse processo podem ocorrer variações comportamentais do fenômeno que, se não forem consideradas, prejudicam os modelos obtidos. Por isso, conforme visto no Capítulo 2 , existem diversos algoritmos de agrupamento que visam adaptar partições de acordo com tais variações.

A adaptação de partições ao longo da coleta de dados é necessária, mas trabalhos existentes não consideram a influência do comportamento variável sobre o viés indutivo estabelecido no projeto de algoritmos de agrupamento de fluxos de dados. Algumas das possíveis consequências do comportamento variável presente em fluxos de dados são: a modificação da relevância de atributos, a inadequação da função de distância utilizada para representar as relações de similaridade entre elementos e o fato do viés de parâmetros tornar-se obsoleto ao longo da coleta de dados.

Recentemente, tem-se buscado alternativas para tratar as consequências de variações de comportamento presentes em fluxos de dados. Pesquisas na área de Meta-aprendizado têm sido dedicadas a esse problema (Brazdil et al., 2009) e têm como objetivo automatizar a escolha do viés indutivo por meio de métodos que aprendem quais linguagens de representação de hipóteses, algoritmos indutivos e parâmetros são adequados de acordo com a descrição dos 
fenômenos observados e dos dados coletados.

As ideias apresentadas em Meta-aprendizado têm começado a refletir na abordagem de problemas causados pela variação de comportamento em fluxos de dados (Brazdil, 2009). Contudo, em um levantamento por abordagens que consideram tais problemas, apenas duas foram encontradas. Ainda assim, essas duas abordagens consideram apenas processos indutivos supervisionados (Brazdil et al., 2009; Pavlidis et al., 2011) e têm como objetivo automatizar a adaptação de viés de preferência de algoritmos de classificação. Elas são baseadas no cálculo de erro em relação à supervisão fornecida por especialistas, a fim de adaptar o viés indutivo de preferência de parâmetros (Pavlidis et al., 2011) e o viés indutivo de preferência de código (Brazdil et al., 2009).

Diferentemente de métodos supervisionados, a indução por meio de agrupamento de dados não conta com a supervisão de especialistas para estabelecer um objetivo a ser alcançado. Portanto, as abordagens propostas para o processo indutivo supervisionado não são diretamente aplicáveis à exploração analítica de fluxos de dados. Dessa forma, observa-se a necessidade de técnicas que adaptem o viés indutivo de algoritmos de agrupamentos de dados, de acordo com variações de comportamentos presentes em fluxos de dados.

\subsection{Proposta da arquitetura de rede neural artificial auto- organizável Self-Organizing Novelty Detector}

Algoritmos incrementais de agrupamento visam adicionar novos elementos a grupos sem a reavaliação de dados prévios (Marsland, 2002; Itti \& Baldi, 2005). Devido à baixa complexidade computacional, esses algoritmos apresentam um grande potencial para aplicação em fluxos de dados. Contudo, atualmente eles apresentam limitações em relação à adaptação de parâmetros de aprendizado de acordo com a ocorrência de variação de comportamento de fenômenos. Essas limitações foram abordadas na proposta da arquitetura de rede neural artificial Self-Organizing Novelty Detector (SONDE) (Albertini \& Mello, 2007), cujo objetivo é representar estados comportamentais do fenômeno por meio da adaptação de estruturas de conhecimento de neurônios. Dessa forma, a arquitetura SONDE constituiu a primeira parte do desenvolvimento da abordagem proposta no objetivo desta tese.

A unidade básica de representação de conhecimento da SONDE é o neurônio, o qual acumula informações históricas sobre elementos, que se denominam padrões de entrada no contexto de redes neurais artificiais. Cada neurônio representa um grupo de padrões similares e é continuamente adaptado de acordo com suas variações. A arquitetura SONDEé composta por três camadas (Figura 4.2): uma camada de entrada para pré-processamento, na qual padrões são opcionalmente normalizados, quando o número de atributos, ou dimensões, $l$ é maior 
que 1; uma camada de armazenamento, na qual neurônios são utilizados para representar as tendências dos padrões de entrada; e a camada competitiva, na qual neurônios disputam para serem eleitos como a unidade mais adequada para representar o padrão observado, ou seja, em inglês, Best-Matching Unit (BMU).

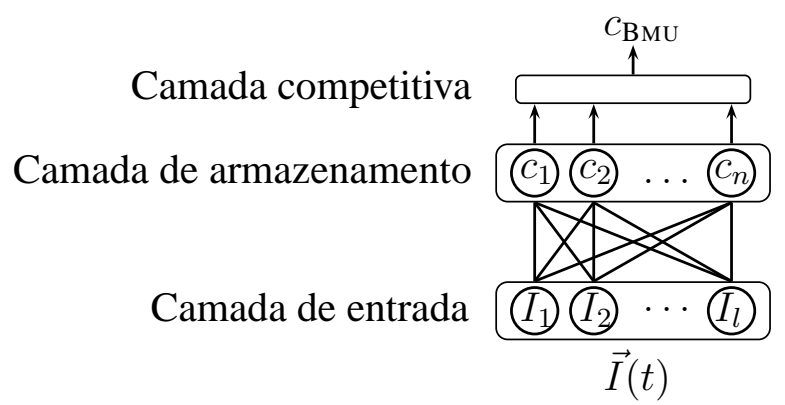

Figura 4.2: Arquitetura de rede neural artificial Self-Organizing Novelty Detector (SONDE)

Seja um padrão de entrada obtido no instante de tempo $t$ um vetor de $l$ dimensões de números reais, ou seja, $\vec{I}(t) \in \mathbb{R}^{l}$. O processamento desse padrão inicia-se na primeira camada de neurônios, a qual recebe as $l$ dimensões simultaneamente e, quando $l>1$, a arquitetura pode ser, opcionalmente, configurada para normalizar o padrão, transformando-o na forma $\vec{I}(t)=\frac{\vec{I}(t)}{\|\vec{I}(t)\|}$, de acordo com a norma euclidiana $\|\vec{I}\|=\sqrt{I_{1}^{2}+\ldots+I_{l}^{2}}$, a qual destaca as relações, em vez da magnitude, entre características do padrão. Na segunda camada, cada neurônio $c_{i}$ recebe da camada anterior o padrão de entrada e é ativado conforme sua afinidade a ele. Por sua vez, cada neurônio transmite seu sinal de ativação à terceira camada que elegerá o neurônio mais representativo para o padrão corrente. Esse processo de ativação ocorre, para cada neurônio $c_{i}, \forall i$ da camada de representação, de acordo com as estruturas de conhecimento $\left\{\vec{w}_{i}, \mathrm{R}_{i}, \alpha_{i}\right\}$, em que $\vec{w}_{i}$ representa a tendência média, ou protótipo, dos padrões de entrada agrupados por $c_{i} ; \mathrm{R}_{i} \in \mathbb{R}^{+}$quantifica a dispersão média dos padrões de entrada em torno do protótipo $\vec{w}_{i}$, ou raio médio; e $\alpha_{i}$ estabelece o limiar de ativação mínimo para um neurônio ser considerado capaz de representar um padrão.

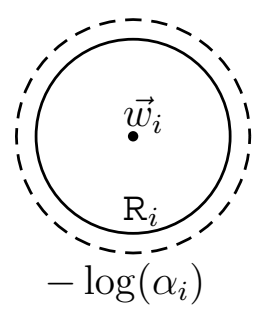

Figura 4.3: Arquitetura SONDE: ilustração de um neurônio $c_{i}$ e suas estruturas de conhecimento

As estruturas de conhecimento de um neurônio $c_{i}$ são adaptadas quando esse é eleito, na camada competitiva, o neurônio mais representativo, ou seja, $c_{\mathrm{BMU}}$. A adaptação de $c_{\mathrm{BMU}}$ 
modifica essas estruturas, i.e., o protótipo $\vec{w}_{\mathrm{BMU}}$, o raio médio $\mathrm{R}_{\mathrm{BMU}}$ e o limiar de ativação $\alpha_{\mathrm{BMU}}$, por meio das Equações 4.1, 4.2, 4.4.

As equações de adaptação utilizam médias móveis exponenciais ponderadas (Roberts, 1966; Winters, 1960; Box \& Jenkins, 1976; Holt, 2004), que provêm adaptação incremental ao conhecimento dos neurônios de acordo com variações presentes nos padrões de entrada. Essas equações atuam por meio dos parâmetros de ponderação $\gamma \in(0,1]$ e $\Omega \in(0,1]$, que caracterizam o aprendizado da tendência atual dos dados compensado por um esquecimento exponencial do passado (Albertini \& Mello, 2008). Essas equações permitem que os neurônios sigam modificações de tendência e comportamento do fenômeno observado. Observe que se define $\vec{w}_{\mathrm{BMU}}(0)=\vec{I}(0)$ e $\mathrm{R}_{\mathrm{BMU}}(0)=-\log (\alpha(0))$.

$$
\begin{gathered}
\vec{w}_{\mathrm{BMU}}(t)=(1-\gamma) \vec{w}_{\mathrm{BMU}}(t-1)+\gamma \vec{I}(t) \\
\mathrm{R}_{\mathrm{BMU}}(t)=(1-\Omega) \mathrm{R}_{\mathrm{BMU}}(t-1)+\Omega\left\|\vec{I}(t)-\vec{w}_{\mathrm{BMU}}(t-1)\right\|
\end{gathered}
$$

A última estrutura de conhecimento do neurônio, isso é, o limiar de ativação $\alpha_{i}$, que define um valor mínimo para a ativação $a_{i}(\vec{I}(t))$ de um neurônio $c_{i}$ (Equação 4.3 ) para que esse seja considerado capaz de representar $\vec{I}(t)$.

$$
a_{i}(\vec{I}(t))=\exp ^{-\left\|\vec{I}(t)-\vec{w}_{i}\right\|}
$$

O limiar de ativação do neurônio $c_{\mathrm{Bmu}}$, i.e., $\alpha_{\mathrm{BMU}}$, é adaptado de acordo com a Equação 4.4, na qual $\theta(t)$ é a taxa de modificação relativa ao raio médio $\mathrm{R}_{\mathrm{BMu}}(t)$ (Equação 4.5).

$$
\begin{gathered}
\alpha_{\mathrm{BMU}}(t)=\min \left\{(1+\theta(t)) \alpha_{\mathrm{BMU}}(t-1), \exp ^{-(1+\theta(t)) \mathrm{R}_{\mathrm{BMU}}(t)}\right\} \\
\theta(t)=\frac{\left|\mathrm{R}_{\mathrm{BMU}}(t)-\mathrm{R}_{\mathrm{BMU}}(t-1)\right|}{\max \left\{\mathrm{R}_{\mathrm{BMU}}(t), \mathrm{R}_{\mathrm{BMU}}(t-1)\right\}}
\end{gathered}
$$

Essa técnica de adaptação para $\alpha_{\mathrm{BMU}}(t)$ visa influenciar o aprendizado de padrões de forma a ponderar a distância entre o raio médio $\mathrm{R}_{\mathrm{BMU}}(t)$, que representa um limite interno para a especialização do neurônio, e a projeção do limiar de ativação $\exp ^{-\alpha_{\text {Bмu }}(t-1)}$. Ela pode adaptar o limiar de ativação a partir de um valor proporcional a $\alpha_{\mathrm{BMU}}(t-1)$, ou seja, o termo $(1+\theta(t)) \alpha_{\mathrm{BMU}}(t-1)$, ou a partir de um valor proporcional ao próprio valor da dispersão média dos padrões de entrada em torno do protótipo do neurônio, ou seja, o termo $\exp ^{-(1+\theta(t)) \mathrm{R}_{\mathrm{Bмu}}(t)}$. Ambos termos são ponderados pela taxa de modificação relativa ao raio médio $\theta(t)$. Entre essas duas opções de especialização para representar padrões de entrada, 
a regra de adaptação do limiar de ativação (Equação 4.4) seleciona aquela que provê a maior generalização aos dados, buscando um equilíbrio no aprendizado. Dessa forma, quanto maior a distância entre o raio médio e o logaritmo do limiar de ativação, mais rápida é a especialização para representar padrões de entradas. Por outro lado, quando padrões são bem distribuídos pela área de cobertura formada por $\alpha_{\mathrm{BMU}}(t)$, a projeção do limiar de ativação e o raio médio tendem a valores similares, indicando convergência.

Na terceira camada da arquitetura, neurônios competem para representar cada padrão. O neurônio $c_{i}$ é eleito o neurônio mais representativo, ou seja, $c_{\mathrm{Bmu}}$, se o protótipo $\vec{w}_{i}(t)$, quando comparado ao padrão de entrada $\vec{I}(t)$, obtiver a maior ativação entre todos os neurônios, de acordo com a Equação 4.3 , e se a ativação obtida $a_{i}(\vec{I}(t))$ for, ainda, maior que o limiar de ativação $\alpha_{i}$.

Caso nenhum neurônio satisfaça as condições de ser eleito $c_{\mathrm{BMU}}$, um novo neurônio é criado. As estruturas de conhecimento desse novo neurônio $c_{\mathrm{BMU}}$ são definidas da seguinte forma: $\vec{w}_{n e w}=\vec{I}(t), \mathrm{R}_{\text {new }}=-\log \left(\alpha_{0}\right)$ e $\alpha_{\text {new }}=\alpha_{0}$, em que $\alpha_{0}$ é uma constante.

Um algoritmo para a implementação da arquitetura SONDE e uma análise de sua complexidade de tempo e espaço são apresentados no Apêndice $\mathrm{A}$ desta tese.

\subsection{Caracterização de comportamento baseada em Entropia de Shannon e em cadeias de Markov}

Como parte da segunda etapa de projeto da abordagem para a adaptação de viés de parâmetros para agrupamento de fluxos de dados, necessita-se de uma medida que avalie variações comportamentais do fenômeno em estudo. Estudos sobre detecção de novidades permitiram observar a importância da utilização de medidas de novidades temporais para a caracterização de comportamento de fenômenos e motivaram a proposta de um método de estimação de cadeias de Markov utilizando a arquitetura SONDE,

Cadeias de Markov são modelos estocásticos que representam relações de causa e efeito entre estados comportamentais:w do fenômeno observado. Um estado é definido por uma conjunção notável de condições do fenômeno observadas a partir dos dados coletados. Por exemplo, um ventilador pode assumir estados em que se observa diferentes velocidades de rotação da hélice: fraco, médio, forte ou desligado. Modelos baseados em cadeias de Markov representam uma grande variedade de fenômenos em que dependências temporais são caracterizadas por transições probabilísticas entre estados. Um sistema típico modelado por cadeias de Markov apresenta estados cujas transições dependem apenas dele mesmo. Dessa forma, no exemplo do ventilador, a transição do estado desligado para qualquer outro é provavelmente relacionada à temperatura do ambiente e independente dos estados anteriores 
ao atual.

Mais formalmente, uma cadeia de Markov é representada por um dígrafo $C=(V, T)$ , em que $V$ é um conjunto de $k$ estados comportamentais e $T$ é um conjunto de arestas com probabilidades associadas a transições entre estados. Essa cadeia pode ser representada por uma matriz de probabilidades de transições $\mathbf{M}$ (Equação 4.6), na qual o peso de uma aresta do conjunto $T$, ou seja, probabilidade de transição de um estado $i$ a outro $j$, corresponde ao elemento $p_{i, j}$ de $\mathbf{M}$, conforme apresentado na Equação 4.6.

$$
\mathbf{M}=\left(\begin{array}{ccc}
p_{1,1} & \ldots & p_{1, j} \\
\vdots & p_{i, j} & \vdots \\
p_{i, 1} & \ldots & p_{n, n}
\end{array}\right)
$$

Com o objetivo de estimar cadeias de Markov a partir do aprendizado da arquitetura SONDE, realiza-se a contagem das transições entre neurônios BMU após a classificação de cada padrão de entrada, ou seja, $\left\{\ldots, c_{\mathrm{BMU}}(t-1), c_{\mathrm{BMU}}(t)\right\}$. Esse processo é um método de estimação de cadeias de Markov baseado em uma amostra longa (Ciuperca \& Girardin, 2007). Nesse contexto, para cada neurônio da SONDE, adiciona-se um estado em $V$. A estimação da matriz de transições $M$ é realizada por meio da contagem do número de ativações ocorridas do neurônio $c_{i}$ seguidas da ativação pelo neurônio $c_{j}$, definida pelo elemento $l_{i, j}$ da matriz $\mathbf{L}$ na Equação 4.7 .

$$
\mathbf{L}=\left(\begin{array}{ccc}
l_{1,1} & \ldots & l_{1, j} \\
\vdots & l_{i, j} & \vdots \\
l_{i, 1} & \ldots & l_{n, n}
\end{array}\right)
$$

Cada elemento da matriz de probabilidades $\mathbf{M}$, ou seja, $p_{i, j}$, é estimado por meio da divisão do elemento $l_{i, j}$ pela soma $l_{i}=\sum_{j=1}^{n} l_{i, j}$ dos elementos da linha $i$ de $\mathbf{L}$, na forma $p_{i, j}=\frac{l_{i, j}}{l_{i}}$. Considerando o aprendizado incremental da SoNDE, para cada novo padrão de entrada no instante de tempo $t$, uma nova cadeia de Markov $\mathbf{M}(t)$ é estimada.

A partir da sequência de cadeias de Markov $\mathbf{M}(t)$ estimadas durante o aprendizado de uma sequência, é possível aplicar medidas de variação de conhecimento (Pincus, 1991), tal como a entropia $\mathbb{H}(t)$ de Shannon (1948), que é obtida a partir de uma matriz de probabilidades $\mathbf{M}(t)$ de acordo com a Equação 4.8 .

$$
\mathbb{H}(t)=-\sum_{i=1}^{n} \sum_{j=1}^{n} p_{i, j}(t) \log \left(p_{i, j}(t)\right)
$$

Ao longo da obtenção de uma sequência de cadeias de Markov, é possível medir a 


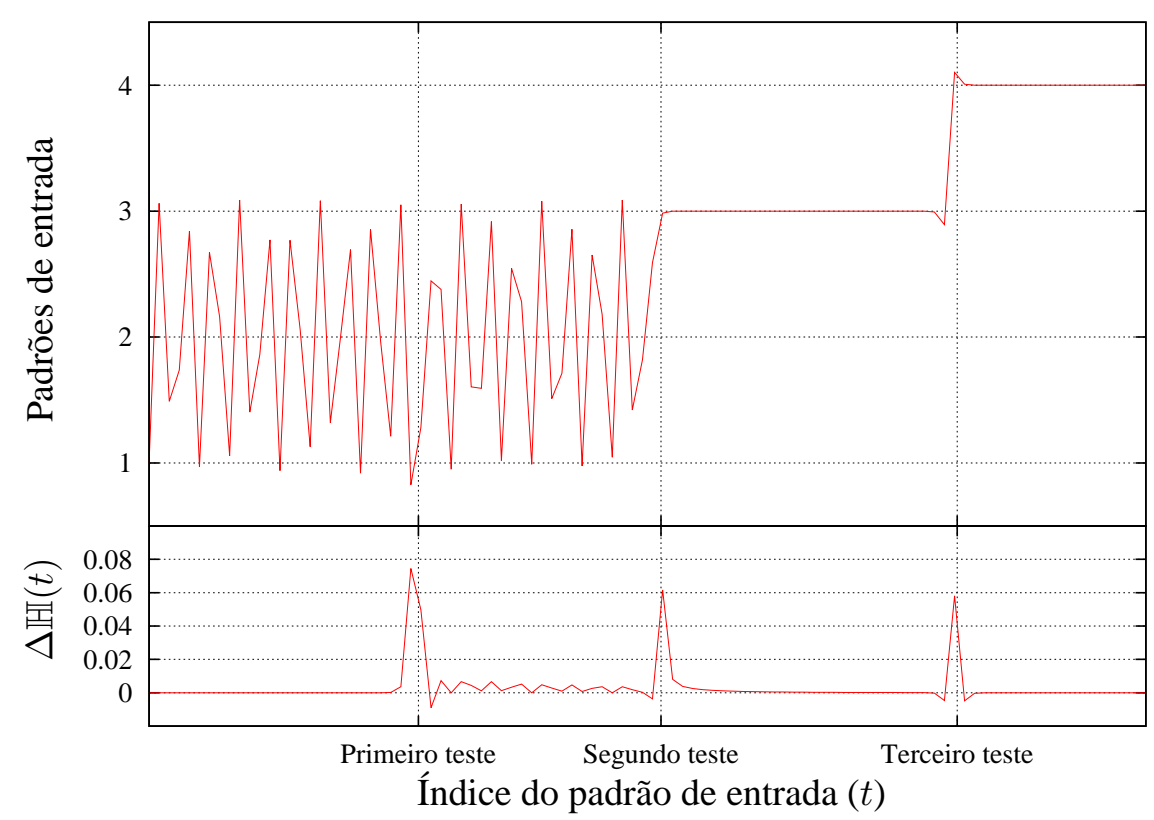

Figura 4.4: Experimento de detecção de novidades em uma sequência periódica com três valores

variação de comportamento em fluxos de dados utilizando a diferença da entropia $\Delta \mathbb{H}(t)$ entre instantes de tempo consecutivos, de acordo a Equação 4.9. A variação de entropia $\Delta \mathbb{H}(t)$ provê uma medida para detectar novidades no comportamento de fluxos de dados e adaptar o viés indutivo do algoritmo de agrupamento. Observe que essa medida também pode ser obtida para qualquer outro algoritmo de agrupamento e não somente para a arquitetura SONDE.

$$
\Delta \mathbb{H}(t)=\mathbb{H}(t)-\mathbb{H}(t-1)
$$

A seguinte simulação foi realizada com o objetivo de exemplificar a detecção de novidades a partir da variação da entropia. Nesse experimento, ilustrado na Figura 4.4 a arquitetura SONDE, parametrizada com $\gamma=0,1, \Omega=0,1$ e $\alpha_{0}=0,4$, recebe a sequência $\{1,2,3,1,2,3, \ldots, 1,1,1,2,3,1,2,3, \ldots, 3,3,3, \ldots, 4,4,4, \ldots\}$ que representa padrões de entrada unidimensionais.

Nesse experimento, três testes foram realizados para exemplificar a detecção e medição de níveis de novidade. O primeiro correspondeu à inserção do elemento 1 , previamente conhecido pela SONDE, contudo em ordem inesperada (após outro 1). Essa inserção não causa a criação de um novo neurônio na SONDE, porém provoca variação de entropia. $\mathrm{O}$ segundo teste tratou da inserção de repetidos valores 3 , que também não provocam a criação de neurônios, mas variam a entropia. Nota-se que, após a apresentação da sequência de valores 3 , a variação de entropia é atenuada, correspondendo a quedas nos níveis de novidade.

Esse teste ilustra a importância do uso de medidas, tais como a entropia, capazes de 
caracterizar a variação de comportamento em termos relativos, ou seja, em níveis, em vez de apenas indicar se houve ou não. Dessa forma, é possível avaliar continuamente o fenômeno em observação. Por último, foram apresentados elementos de valor 4, até então desconhecidos, os quais provocam a criação de um novo neurônio e, ainda, implicam na variação de entropia devido ao aumento de estados e alteração das probabilidades de transição da cadeia de Markov.

Em geral, nesse experimento foi possível perceber que a detecção de novidades somente baseada em medidas espaciais, ou seja, na criação de novos neurônios não é suficiente, pois não é capaz de utilizar informações implícitas na sequência de coleta dos padrões de entrada para indicar novidades temporais (quando o 1 e 3 apareceram em momento inesperado). Adicionalmente, graças à variação de entropia observada no segundo teste, pode-se realizar um paralelo de sua atenuação com a redução do nível de novidade devido ao aprendizado do novo comportamento, observado pela sequência de valores 3 .

Em redes neurais artificiais auto-organizáveis, tais como SOM GWR algoritmos que tratam fluxos de dados como conjuntos tradicionais, esse tipo de variação de comportamento não seria detectado, pois esses algoritmos consideram apenas aspectos espaciais e não aspectos relativos à sequência de coleta. Por outro lado, algoritmos que permitem a utilização de medidas de novidades temporais, conforme apresentado no Capítulo 3, são adequados para a avaliação de comportamento de fenômenos e adaptação do viés indutivo.

\subsection{Primeira abordagem para a adaptação de viés indutivo}

A primeira abordagem para adaptação de viés de parâmetros foi motivada pelo uso da medida de entropia para a detecção de variação de comportamento de fenômenos. Essa abordagem assume que a variação de entropia é um produto de modificações de agrupamentos presentes em fluxos de dados e da inadequação do viés de parâmetros em permitir que o algoritmo de agrupamento represente adequadamente as variações de comportamento do fenômeno em estudo. O objetivo da primeira abordagem foi o de atribuir e corrigir as inadequações do viés indutivo a partir de um método de otimização baseado em gradiente descendente, conforme utilizado em redes neurais com treinamento baseado em retropropagação de erros (Werbos, 1993). O método baseado em gradiente descendente visa otimizar uma função de custo, conhecida como função-objetivo, por meio de uma busca orientada pela direção de maior variação da função, ou seja, o seu gradiente.

Para o desenvolvimento dessa abordagem, primeiramente, observou-se que toda variação de entropia em cadeias de Markov está associada a uma transição de um estado passado $X_{i}(t-1)$ para o estado atual $X_{j}(t)$. A modificação que ocorreu no tempo $t$ no valor da entropia $\mathbb{H}(t)$ é medida por meio da Equação 4.10, assumindo que $\mathbb{H}(t)$ seja uma função contínua com amostras periódicas. Nesse contexto, define-se o número de estados na cadeia no instante de 
tempo $t$ como $k(t)$.

$$
\frac{\partial \mathbb{H}_{i}(t)}{\partial t}=-\sum_{h=1}^{k(t)} \frac{\partial\left[p_{i, h} \log _{2}\left(p_{i, h}\right)\right]}{\partial t}
$$

A adequação do viés de parâmetros desta primeira abordagem é conduzida por uma função-objetivo que relaciona a variação de entropia $\frac{\partial \mathbb{H}_{i}(t)}{\partial t}$ e parâmetros do viés indutivo. Uma vez que se considera a arquitetura SONDE para o agrupamento de fluxos de dados, deve-se atualizar seus parâmetros $\gamma_{j}$ e $\Omega_{j}$ a fim de adaptar seu viés indutivo em relação às variações comportamentais do fenômeno em estudo. Para isso derivou-se como função-objetivo $f\left(\gamma_{j}, \Omega_{j}, t\right)$ (Equação 4.11), na qual $p_{i, h}$ depende de $\gamma_{j}$ e $\Omega_{j}$ (definidos nas Equações 4.1 e 4.2). Essa função-objetivo representa a variação de entropia induzida pelos parâmetros do neurônio atual $c_{j}$, que corresponde ao estado $X_{j}$.

$$
f\left(\gamma_{j}, \Omega_{j}, t\right)=\frac{\partial \mathbb{H}_{i}(t)}{\partial t}=-\sum_{h=1}^{k(t)}\left[\frac{1}{\log 2} \frac{\partial p_{i, h}}{\partial t}\left(\log _{2}\left(p_{i, h}\right)+1\right)\right]
$$

A função-objetivo $f\left(\gamma_{j}, \Omega_{j}, t\right)$ deve ser minimizada durante o processo de aprendizado por meio de $\gamma_{j}$ e $\Omega_{j}$, considerando os parâmetros $\gamma_{j} \in(0,1]$ e $\Omega_{j} \in(0,1]$. Portanto, nesta abordagem, considera-se que cada neurônio $c_{i}$ tem seus parâmetros individuais $\gamma_{i}$ e $\Omega_{i}$.

O desenvolvimento desta abordagem consistiu em uma etapa de análise de convergência de aprendizado (Seção 4.6.1), na qual se procurou identificar propriedades de convergência de aprendizado com o objetivo de descrever a relação entre $p_{i, j}$ e os parâmetros $\gamma_{j}$ e $\Omega_{j}$, uma etapa de obtenção de um vetor-gradiente a partir das propriedades de convergência (Seção 4.6.2) e uma etapa de estudos sobre a orientação das buscas para otimização da função-objetivo, de acordo com as restrições dos valores de $\gamma_{j}$ e $\Omega_{j}$ (Seção 4.6.3).

\subsubsection{Análise da convergência de aprendizado}

Com o objetivo de adaptar o viés indutivo da arquitetura SONDE, deve-se minimizar $f\left(\gamma_{j}, \Omega_{j}, t\right)$ a partir da relação entre as probabilidades $p_{i, j}$ e os parâmetros $\gamma_{j}$ e $\Omega_{j}$. Porém, devido ao desconhecimento de relações exatas entre essas probabilidades, que dependem do fluxo de dados com comportamento variável, e os parâmetros mencionados, foi necessário buscar por propriedades de convergência para as equações de adaptação das estruturas de conhecimento da arquitetura SONDE, Tais propriedades de convergência, obtidas a partir da premissa de que é provável a observação de sequências de padrões suficientemente longas e estáveis, descrevem relações entre probabilidades e os parâmetros independentemente de conhecimento sobre o comportamento dos padrões de entrada $\vec{I}(t)$, coletados a partir da observação do fenômeno em estudo. 
Inicialmente, representou-se a probabilidade $p_{i, j}$ em termos da ativação neural proveniente da Equação 4.12, com $a_{j}(\vec{I}(t))$ (Equação 4.3) sendo o nível de ativação de um padrão de entrada $\vec{I}(t)$ para o neurônio $c_{j}$ com um nível mínimo de similaridade $\alpha_{j}(t)$, no instante de tempo $t$. Após a convergência de aprendizado, raramente se espera competição entre dois neurônios por um padrão. Define-se convergência de aprendizado quando a ativação de $c_{j}$ é certamente a mais alta, i.e., $\operatorname{Pr}\left(\left\{a_{j}(t) \geq a_{h}(t), \forall c_{h}\right\}\right) \approx 1$. Nessas condições, $p_{i, j}$ tende ${ }^{4}$ a $p_{i, j}^{\infty}$ (Equação 4.13).

$$
\begin{gathered}
p_{i, j}=\operatorname{Pr}\left(X(t)=c_{j} \mid X(t)=c_{i}\right)=\operatorname{Pr}\left(\left\{a_{j}(\vec{I}(t))>\alpha_{j}(t)\right\} \cap\left\{a_{j}(t)>a_{h}(t), \forall h\right\}\right) \\
p_{i, j}^{\infty}=\operatorname{Pr}\left(a_{j}(\vec{I}(t)) \geq \alpha_{j}(t)\right) \approx p_{i, j}
\end{gathered}
$$

Para definir a função de distribuição de probabilidades de $p_{i, j}^{\infty}$, buscou-se descrever a relação entre a ativação do neurônio $c_{j}$, sua equação de adaptação e o nível mínimo de similaridade $\alpha_{j}(t)$. Essa relação depende das estruturas de conhecimento do neurônio $c_{j}$, i.e., o protótipo $\vec{w}_{j}(t)$, que utiliza o parâmetro $\gamma_{j}$ (Equação 4.1) para sua adaptação, e o raio médio $\mathrm{R}_{j}(t)$, obtido em função do parâmetro $\Omega_{j}$ (Equação 4.2). Observe que ambos parâmetros são idealmente definidos de acordo com a necessidade de adaptação do conhecimento ao longo do tempo.

No passo seguinte, analisou-se a convergência de $\mathrm{R}(t)$ ao aplicar a equação de adaptação do protótipo (Equação 4.1) na adaptação de raio (Equação 4.2):

$$
\mathrm{R}(t)=\left(1-\Omega_{j}\right) \mathrm{R}(t-1)+\Omega_{j}\left\|\vec{I}(t)-\left(1-\gamma_{j}\right) \vec{w}(t-2)-\gamma_{j} \vec{I}(t-1)\right\|
$$

Considerando que padrões similares são representados pelo mesmo protótipo em uma instância treinada da arquitetura da SONDE, observa-se que $\mathrm{R}(t)$ converge para $\mathrm{R}^{\infty}(t)$ quando as entradas nos instantes $t$ e $t-1$ se aproximam, i.e., quando a sequência de padrões representada pelo mesmo protótipo é similar:

$$
\begin{array}{r}
\mathrm{R}^{\infty}(t)=\left(1-\Omega_{j}\right) \mathrm{R}^{\infty}(t-1)+\Omega_{j}\left\|\left(1-\gamma_{j}\right) \vec{I}(t-1)-\left(1-\gamma_{j}\right) \vec{w}(t-2)\right\| \\
=\left(1-\Omega_{j}\right) \mathrm{R}^{\infty}(t-1)+\Omega_{j}\left(1-\gamma_{j}\right)\|\vec{I}(t-1)-\vec{w}(t-2)\|
\end{array}
$$

Neste ponto, é possível substituir o termo correspondente ao padrão de entrada $\vec{I}(t-$

\footnotetext{
${ }^{4}$ As variáveis marcadas com $\infty$ são aproximações ou resultados de condições de convergência.
} 
1), que segue uma função de distribuição de probabilidades desconhecida, com medidas de dispersão em torno do protótipo $\mathrm{R}^{\infty}(t-1) \approx\|\vec{I}(t-1)-\vec{w}(t-2)\|$. Essa substituição é razoável uma vez que $\mathrm{R}(t)$ é um estimador para o valor esperado $E(\|\vec{I}(t-1)-\vec{w}(t-2)\|)$, assim como o é para $E(\|\vec{I}(t)-\vec{w}(t-1)\|)$, e, além disso, $c_{j}$ é o neurônio $c_{\mathrm{BMU}}$ para $\vec{I}(t-1)$. Portanto, obtém-se a Equação 4.14 que resume a tendência da especialização de raio considerando os parâmetros passíveis de alteração, i.e., $\gamma_{j}$ e $\Omega_{j}$.

$$
\mathrm{R}^{\infty}(t)=\left(1-\Omega_{j} \gamma_{j}\right) \mathrm{R}^{\infty}(t-1)
$$

A convergência de aprendizado também afeta o nível mínimo de similaridade $\alpha(t)$ (Equação 4.4), regulado pela medida de especialização $\theta(t)$ (Equação 4.5). O processo de aprendizado inicia-se com um nível mínimo de similaridade $\alpha_{0}$ e, para cada nova entrada $\overrightarrow{(t)}$, ele, por definição, segue o valor mais baixo entre o limiar de ativação $\alpha(t-1)(1+\theta(t))$ e a variação média dos padrões de entrada $\mathrm{R}(t-1)(1+\theta(t))$.

Durante a especialização de $\alpha(t)$, se $(1+\theta(t)) \cdot \alpha(t-1)<\exp ^{-\mathrm{R}(t)(1+\theta(t))}$, então $\mathrm{R}(t)$ convergirá para a variação dos padrões restritos por $\alpha(t)$. Se $\alpha(t-1)(1+\theta(t))>$ $\exp ^{-\mathrm{R}(t)(1+\theta(t))}$, então $\alpha(t)$ assumirá $\exp ^{-\mathrm{R}(t)(1+\theta(t))}$. Portanto, o termo $\alpha(t)$ se aproximará do raio de dispersão $\mathrm{R}(t)$, conforme apresentado na Equação 4.15 ,

$$
\alpha(t) \approx \alpha(t-1)(1+\theta(t)) \approx \exp ^{-\mathrm{R}(t)(1+\theta(t))}
$$

Considerando que $\gamma_{j}$ e $\Omega_{j}$ são positivos, verifica-se a partir da Equação 4.14 que $\mathrm{R}^{\infty}(t-1)>\mathrm{R}^{\infty}(t)$ e $\frac{\mathrm{R}^{\infty}(t)}{\mathrm{R}^{\infty}(t-1)}=1-\gamma_{j} \Omega_{j}$, o que permite o uso de $\mathrm{R}^{\infty}(t)$ para simplificar a medida de especialização $\theta(t)$ em $\theta^{\infty}(t)$, conforme a Equação 4.16, e aplicá-la para obter $\alpha^{\infty}(t)$, conforme definido na Equação 4.17.

$$
\begin{gathered}
\theta(t)=\frac{|\mathrm{R}(t)-\mathrm{R}(t-1)|}{\max \{\mathrm{R}(t), \mathrm{R}(t-1)\}} \\
\theta^{\infty}(t) \approx\left|\frac{\mathrm{R}^{\infty}(t)-\mathrm{R}^{\infty}(t-1)}{\mathrm{R}^{\infty}(t-1)}\right|=\left|\frac{\mathrm{R}^{\infty}(t)}{\mathrm{R}^{\infty}(t-1)}-1\right|=\left|1-\gamma_{j} \Omega_{j}-1\right|=\gamma_{j} \Omega_{j} \\
\alpha^{\infty}(t) \approx \alpha(t) \approx \exp ^{-\mathrm{R}(t)(1+\theta(t))} \approx \exp ^{-\mathrm{R}^{\infty}(t)\left(1+\theta^{\infty}(t)\right)}=\exp ^{-\mathrm{R}^{\infty}(t-1)\left(1-\gamma_{j}^{2} \Omega_{j}^{2}\right)}
\end{gathered}
$$

Aplica-se o limiar de ativação mínimo convergido $\alpha^{\infty}(t)$ (Equação 4.17) e a ativação do neurônio (Equação 4.3) na definição da probabilidade $p_{i, j}^{\infty}$ (Equação 4.13) para obter a probabilidade assintótica $p_{i, j}^{\infty}$ (Equação 4.18). Essas probabilidades definem a aceitação de padrões por parte do neurônio $c_{j}$ como relacionada ao aprendizado do raio $\mathrm{R}(t)$, sob a influência 
dos parâmetros $\gamma_{j}$ e $\Omega_{j}$. A sequência dos padrões de entrada representada pelo neurônio $c_{j}$ irá gradualmente se especializar, o que levará à redução de $\mathrm{R}(t)$ em torno do protótipo $\vec{w}_{j}$, de acordo com a Equação 4.14 .

$$
p_{i, j}^{\infty}=\operatorname{Pr}\left(\left\|\vec{I}(t)-\vec{w}_{j}(t-1)\right\|<\mathrm{R}(t)\left(1-\gamma_{j}^{2} \Omega_{j}^{2}\right)\right)
$$

\subsubsection{Obtenção de um vetor-gradiente da superfície do viés de parâme- tros}

Após as etapas de formalização e definição de propriedades de convergência simplificadoras, obtém-se, nesta seção, um método de otimização da função-objetivo (Equação 4.10) utilizando probabilidades assintóticas $p_{i, j}^{\infty}$ descritas na seção anterior (Equação 4.18). A otimização da função-objetivo busca adaptar os parâmetros da rede SoNDEde acordo com a parcela da variação de entropia atribuída ao comportamento do fenômeno observado.

Para obter as equações de otimização da função-objetivo, primeiramente, organizam-se as probabilidades assintóticas $p_{i, j}^{\infty}$ para a Equação 4.19 utilizando $\phi\left(\gamma_{j}, \Omega_{j}, t\right)$ (Equação 4.20), ou, para simplificar a notação $\phi=\phi\left(\gamma_{j}, \Omega_{j}, t\right)$, como uma função da dispersão de padrões $\mathrm{R}(t)$ e parâmetros $\gamma_{j}$ e $\Omega_{j}$.

$$
\begin{gathered}
p_{i, j}^{\infty}=\operatorname{Pr}\left(Y<\phi\left(\gamma_{j}, \Omega_{j}, t\right)\right) \\
\phi\left(\gamma_{j}, \Omega_{j}, t\right)=\left(1-\gamma_{j}^{2} \Omega_{j}^{2}\right) \mathrm{R}(t)
\end{gathered}
$$

A seguir, observa-se que $Y=\left\|\vec{I}(t)-\vec{w}_{j}(t-1)\right\|$ representa a distância entre um protótipo $\vec{w}_{j}$ e seus padrões correspondentes $\vec{I}(t)$. Devido ao fato que um protótipo $\vec{w}_{j}(t)$ deve aproximar uma sequência de padrões de entrada $\vec{I}(t)$ representados por $c_{j}, Y$ é considerada ter um comportamento de erro e, portanto, assume-se que é descrita de acordo com uma função de distribuição de probabilidades Normal $Y \sim N\left(\mu, \sigma^{2}\right)$. Para simplificar a notação, sem prejuízo à obtenção do gradiente da função-objetivo, considera-se a média de erros $\mu=0$ e assume-se que $y$ possa assumir valores negativos. Dessa forma, as probabilidades para os valores observados de $y=\phi$ são representados pela Equação 4.21, que é descrita em termos da função de erro erf $(y)$, apresentada na Equação 4.22 (Shefler, 1988).

$$
\operatorname{Pr}(Y<y)=\frac{1}{\sigma \sqrt{2 \pi}} \int_{-\infty}^{y} \exp \left(-\frac{(y-\mu)^{2}}{2 \sigma^{2}}\right)=\frac{1}{2}\left(1+\operatorname{erf}\left(\frac{y-\mu}{\sigma \sqrt{2}}\right)\right)
$$




$$
\operatorname{erf}(y)=\frac{2}{\sqrt{\pi}} \int_{-\infty}^{y} \exp ^{-u^{2}} d u
$$

Quando a distribuição cumulativa Normal $\operatorname{Pr}(Y<y)$ é aplicada às condições da equação de probabilidade assintótica $p_{i, j}^{\infty}$, tem-se:

$$
p_{i, j}^{\infty}=\frac{1}{2}+\frac{1}{\sqrt{\pi}} \int_{-\infty}^{\frac{\phi\left(\gamma_{j}, \Omega_{j}, t\right)}{\sigma \sqrt{2}}} \exp ^{-u^{2}} d u=\frac{1}{2}\left(1+\operatorname{erf}\left(\frac{\phi\left(\gamma_{j}, \Omega_{j}, t\right)}{\sigma \sqrt{2}}\right)\right)
$$

A Equação 4.23 é derivada com o intuito de determinar a direção de variação máxima da entropia conforme estabelecido pela função-objetivo na Equação 4.10, por meio da qual se realiza a adaptação de viés de parâmetros da arquitetura SONDE caracterizado por $\gamma_{j}$ e $\Omega_{j}$. Essas derivadas compõem o vetor-gradiente $\nabla f\left(\gamma_{j}, \Omega_{j}, t\right)=\nabla \frac{\partial \mathbb{H}(t)}{\partial t}$ (Equação 4.24), definido pelos componentes nas Equações 4.25 e 4.26, com o objetivo de orientar a adaptação incremental de parâmetros e adequá-los à variação de comportamento do fenômeno em estudo.

$$
\begin{gathered}
\nabla \frac{\partial \mathbb{H}(t)}{\partial t}=\left(\frac{\partial^{2} \mathbb{H}(t)}{\partial \gamma_{j} \partial t}, \frac{\partial^{2} \mathbb{H}(t)}{\partial \Omega_{j} \partial t}\right) \\
\frac{\partial^{2} \mathbb{H}(t)}{\partial \gamma_{j} \partial t}=-\sum_{h=1}^{k(t)} \frac{\partial\left[\frac{1}{\log 2} \frac{\partial p_{i, h}^{\infty}}{\partial t}\left(\log _{2}\left(p_{i, h}^{\infty}\right)+1\right)\right]}{\partial \gamma_{j}} \\
\frac{\partial^{2} \mathbb{H}(t)}{\partial \Omega_{j} \partial t}=-\sum_{h=1}^{k(t)} \frac{\partial\left[\frac{1}{\log 2} \frac{\partial p_{i, h}^{\infty}}{\partial t}\left(\log \left(p_{i, h}^{\infty}\right)+1\right)\right]}{\partial \Omega_{j}}
\end{gathered}
$$

O desenvolvimento dessas equações segue-se a partir da expansão dos termos relativos às probabilidades assintóticas $p_{i, j}^{\infty}$ para obter a Equação 4.27 .

$$
\begin{aligned}
\frac{\partial p_{i, j}^{\infty}\left(\gamma_{j}, \Omega_{j}, t\right)}{\partial t} & =\frac{\partial}{\partial t} \frac{1}{\sqrt{\pi}} \int_{-\infty}^{\frac{\phi}{\sigma \sqrt{2}}} \exp ^{-u^{2}} d u \\
& =\frac{1}{\sqrt{\pi}} \exp ^{-\left(\frac{\phi}{\sigma \sqrt{2}}\right)^{2}} \frac{\partial \mathrm{R}(t)}{\partial t}\left(1-\gamma_{j}^{2} \Omega_{j}^{2}\right)
\end{aligned}
$$

A seguir, ao aplicar a regra de derivada de produtos nos termos das Equações 4.25 e 4.26, que dependem de $\gamma_{j}$ e $\Omega_{j}$, obtêm-se as Equações 4.28 e 4.29 para representar o vetor-gradiente.

$$
\frac{\partial^{2} \mathbb{H}_{i, j}(t)}{\partial \gamma_{j} \partial t}=\frac{1}{\log 2}\left[\frac{\partial^{2} p_{i, j}^{\infty}}{\partial \gamma_{j} \partial t}\left(\log _{2}\left(p_{i, j}^{\infty}\right)+1\right)+\frac{\partial p_{i, j}^{\infty}}{\partial t} \frac{\partial \log _{2}\left(p_{i, j}^{\infty}\right)}{\partial \gamma_{j}}\right]
$$




$$
\frac{\partial^{2} \mathbb{H}_{i, j}(t)}{\partial \Omega_{j} \partial t}=\frac{1}{\log 2}\left[\frac{\partial^{2} p_{i, j}^{\infty}}{\partial \Omega_{j} \partial t}\left(\log _{2}\left(p_{i, j}^{\infty}\right)+1\right)+\frac{\partial p_{i, j}^{\infty}}{\partial t} \frac{\partial \log _{2}\left(p_{i, j}^{\infty}\right)}{\partial \Omega_{j}}\right]
$$

Deve-se, ainda, expandir cada termo dependente de derivadas presentes nas Equações 4.28 e 4.29. Para isso, primeiramente, obtêm-se os termos $\frac{\partial^{2} p_{i, j}^{\infty}\left(\gamma_{j}, \Omega_{j}, t\right)}{\partial \gamma_{j} \partial t}$ (Equação 4.30) e $\frac{\partial^{2} p_{i, j}^{\infty}\left(\gamma_{j}, \Omega_{j}, t\right)}{\partial \Omega_{j} \partial t}$ (Equação 4.31), considerando que $\Delta \phi(t)=\Delta \phi\left(\gamma_{j}, \Omega_{j}, t\right)=\Delta \mathrm{R}(t)\left(1-\gamma_{j}^{2} \Omega_{j}^{2}\right)$, na qual $\Delta \mathrm{R}(t)=\mathrm{R}(t)-\mathrm{R}(t-1)$ :

$$
\begin{aligned}
\frac{\partial^{2} p_{i, j}^{\infty}\left(\gamma_{j}, \Omega_{j}, t\right)}{\partial \gamma_{j} \partial t} & =\frac{\partial}{\partial \gamma_{j}} \frac{1}{\sqrt{\pi}} \exp ^{-\left(\frac{\phi}{\sigma \sqrt{2}}\right)^{2} \Delta \phi(t)} \\
& =\frac{2 \gamma_{j} \Omega_{j}^{2} \Delta \mathrm{R}(t)}{\sqrt{\pi}}\left[\left(\frac{\phi^{2}}{\sigma^{2}}-1\right) \exp ^{\left.-\left(\frac{\phi}{\sigma \sqrt{2}}\right)^{2}\right]}\right. \\
\frac{\partial^{2} p_{i, j}^{\infty}\left(\gamma_{j}, \Omega_{j}, t\right)}{\partial \Omega_{j} \partial t} & =\frac{2}{\sqrt{\pi}} \gamma_{j}^{2} \Omega_{j} \Delta \mathrm{R}(t)\left[\left(\frac{\phi^{2}}{\sigma^{2}}-1\right) \exp ^{-\left(\frac{\phi}{\sigma \sqrt{2}}\right)^{2}}\right]
\end{aligned}
$$

Isso também é realizado para $\frac{\partial \log \left(p_{i, j}^{\infty}\left(\gamma, \Omega_{j}, t\right)\right)}{\partial \gamma}$ (Equação 4.32) e $\frac{\partial \log \left(p_{i, j}^{\infty}\left(\gamma, \Omega_{j}, t\right)\right)}{\partial \Omega_{j}}$ (Equação 4.33).

$$
\begin{aligned}
\frac{\partial \log _{2}\left(p_{i, j}^{\infty}\right)}{\partial \gamma_{j}} & =\frac{\left(\frac{1}{\sqrt{\pi}}\right) \cdot\left(-\frac{\mathrm{R}(t)}{\sigma \sqrt{2}}\right) 2 \gamma_{j} \Omega_{j}^{2} \exp ^{-\left(\frac{\phi}{\sigma \sqrt{2}}\right)^{2}}}{p_{i, j}^{\infty}} \\
& =-\frac{\sqrt{2} \mathrm{R}(t) \gamma_{j} \Omega_{j}^{2} \exp ^{2}\left(\frac{\phi}{\sigma \sqrt{2}}\right)^{2}}{p_{i, j}^{\infty} \sigma \sqrt{\pi}} \\
\frac{\partial \log _{2}\left(p_{i, j}^{\infty}\right)}{\partial \Omega_{j}} & =-\frac{\sqrt{2} \mathrm{R}(t) \gamma_{j}^{2} \Omega_{j} \exp ^{-\left(\frac{\phi}{\sigma \sqrt{2}}\right)^{2}}}{p_{i, j}^{\infty} \sigma \sqrt{\pi}}
\end{aligned}
$$

Com esses termos desenvolvidos, pode-se obter os componentes do vetor-gradiente (Equação 4.34) apresentados nas Equações 4.35 e 4.36, Esses componentes permitem adaptar os parâmetros $\gamma_{j}$ e $\Omega_{j}$ do neurônio $c_{j}$ e, dessa maneira acompanhar a variação de comportamento presente no fluxo de dados.

$$
\begin{gathered}
\nabla \frac{\partial \mathbb{H}_{i, j}(t)}{\partial t}=\left(\frac{\partial^{2} \mathbb{H}_{i, j}(t)}{\partial \gamma_{j} \partial t}, \frac{\partial^{2} \mathbb{H}_{i, j}(t)}{\partial \Omega_{j} \partial t}\right) \\
\frac{\partial^{2} \mathbb{H}_{i, j}(t)}{\partial \gamma_{j} \partial t}=\gamma_{j} \Omega_{j}^{2} \Delta \mathrm{R}(t) \frac{2 \exp -\left(\frac{\phi}{\sigma \sqrt{2}}\right)^{2}}{\log 2 \sqrt{\pi}}\left[\begin{array}{r}
\left(\frac{\phi^{2}}{\sigma^{2}}-1\right)\left(\log _{2}\left(p_{i, j}^{\infty}\right)+1\right) \\
\left.-\frac{\sqrt{2} \phi}{\sqrt{\pi}} \frac{\exp -\left(\frac{\phi}{\sigma \sqrt{2}}\right)^{2}}{2 p_{i, j}^{\infty} \sigma}\right]
\end{array}\right.
\end{gathered}
$$




$$
\begin{aligned}
& \frac{\partial^{2} \mathbb{H}_{i, j}(t)}{\partial \Omega_{j} \partial t}=\gamma_{j}^{2} \Omega_{j} \Delta \mathrm{R}(t) \frac{2 \exp -\left(\frac{\phi}{\sigma \sqrt{2}}\right)^{2}}{\log 2 \sqrt{\pi}} \quad\left[\left(\frac{\phi^{2}}{\sigma^{2}}-1\right)\left(\log _{2}\left(p_{i, j}^{\infty}\right)+1\right)\right. \\
& \left.-\frac{\sqrt{2} \phi}{\sqrt{\pi}} \frac{\exp ^{-\left(\frac{\phi}{\sigma \sqrt{2}}\right)^{2}}}{2 p_{i, j}^{\infty} \sigma}\right]
\end{aligned}
$$

A regra de adaptação de $\gamma_{j}$ e $\Omega_{j}$, apresentada na Equação 4.37, utiliza o vetor-gradiente $\nabla \frac{\partial \mathbb{H}_{i, j}(t)}{\partial t}$ ponderado pelo fator $\eta \in \mathbb{R}^{+}$, o qual define uma taxa de aprendizado para a adaptação de viés de parâmetros de maneira incremental. O intuito dessa regra de adaptação é o de induzir a melhora do processo de agrupamento.

$$
\left(\gamma_{j}(t), \Omega_{j}(t)\right)=\left(\gamma_{j}(t-1), \Omega_{j}(t-1)\right)-\eta \nabla \frac{\partial \mathbb{H}_{i, j}(t)}{\partial t}
$$

Conforme previamente mencionado, a variação de entropia é atribuída parte à inadequação do viés indutivo e parte à desatualização do agrupamento em função do comportamento presente no fluxo de dados. O uso dessa regra de adaptação do viés indutivo busca atenuar a variação de entropia originada a partir da inadequação dos parâmetros do algoritmo de agrupamento.

\subsubsection{Restrições da adaptação do viés indutivo}

Faz-se necessária a definição de restrições para a adaptação do viés indutivo da arquitetura SONDE, uma vez que seus parâmetros $\gamma_{j}$ e $\Omega_{j}$ são definidos no intervalo $(0,1]$. Sem essas restrições, $\gamma_{j}$ ou $\Omega_{j}$ poderiam ser modificados a zero, fazendo com que novos padrões fossem ignorados, ou então, caso atualizados a valores negativos, com que um protótipo ou raio médio siga tendência oposta à do comportamento atual do fenômeno.

Porém, o uso da regra de adaptação, Equação 4.37 não garante respeito a tais restrições. Com o objetivo de garantir $\left(\gamma_{j, t-1}, \Omega_{j, t-1}\right)-\eta \nabla \frac{\partial \mathbb{H}_{i, j}(t)}{\partial t}>0$, ou seja, que os parâmetros atualizados sejam positivos, obtém-se um fator de compensação $\lambda \in \mathbb{R}^{+}$que é composto com $\eta$ da forma $\eta^{*}=\lambda \cdot \eta$ para manter a direção e reduzir a magnitude de adaptação.

O vetor-gradiente $\nabla \frac{\partial \mathbb{H}_{i, j}(t)}{\partial t}=\nabla f\left(\gamma_{j}, \Omega_{j}, t\right)=\left(\frac{\partial f}{\partial \gamma_{j}}, \frac{\partial f}{\partial \Omega_{j}}\right)$ pode ter um ou ambos componentes negativos. Portanto, para tratar tal questão, divide-se o problema em três cenários: quando i) o gradiente é positivo, ii) apenas um componente é negativo, ou iii) ambos são negativos.

O processo de adaptação segue as Equações 4.38 e 4.39. Durante o processo, $\eta, \Omega_{j} \mathrm{e}$ $\gamma_{j}$ devem ser sempre positivos. O valor de $\lambda$ é obtido de acordo com $\frac{\partial f}{\partial \gamma_{j}}$ e $\frac{\partial f}{\partial \Omega_{j}}$. Quando $\frac{\partial f}{\partial \Omega_{j}}$ é negativo, escreve-se como ${\frac{\partial f}{\partial \Omega_{j}}}^{-}$, caso contrário, quando positivo, utiliza-se ${\frac{\partial f}{\partial \Omega_{j}}}^{+}$. 


$$
\begin{aligned}
\gamma_{j}(t+1) & =\gamma_{j}(t)-\lambda \cdot \eta \frac{\partial f}{\partial \gamma_{j}} \\
\Omega_{j}(t+1) & =\Omega_{j}(t)-\lambda \cdot \eta \frac{\partial f}{\partial \Omega_{j}}
\end{aligned}
$$

O primeiro cenário, apresentado nas Equações 4.40 e 4.41, tem ambos os componentes do vetor-gradiente positivos. Portanto, o intervalo da solução para $\lambda$, quando $\frac{\partial f}{\partial \gamma_{j}}>0 \mathrm{e}$ $\frac{\partial f}{\partial \Omega_{j}}>0$, é definido por $\lambda<\min \left\{\frac{\gamma_{j}(t)}{\eta \frac{\partial f}{\partial \gamma_{j}}+}, \frac{\Omega_{j}(t)}{\eta \frac{\partial f}{\partial \Omega_{j}}+}\right\}$. Esse cenário é solucionado por $\lambda=$ $\frac{1}{2} \min \left\{\frac{\gamma(t)}{\eta \frac{\partial f}{\partial \gamma_{j}}}+\frac{\Omega_{j}(t)}{\eta \frac{\partial f}{\partial \Omega_{j}}}+\right.$.

$$
\begin{gathered}
\gamma_{j}(t)-\lambda \cdot \eta{\frac{\partial f}{\partial \gamma_{j}}}^{+}>0 \Rightarrow-\lambda>-\frac{\gamma_{j}(t)}{\eta \frac{\partial f}{\partial \gamma_{j}}} \Rightarrow \lambda<\frac{\gamma_{j}(t)}{\eta{\frac{\partial f}{\partial \gamma_{j}}}^{+}} \\
\Omega_{j}(t)-\lambda \cdot \eta{\frac{\partial f}{\partial \Omega_{j}}}^{+}>0 \Rightarrow-\lambda>-\frac{\Omega_{j}(t)}{\eta{\frac{\partial f}{\partial \Omega_{j}}}^{+}} \Rightarrow \lambda<\frac{\Omega_{j}(t)}{\eta{\frac{\partial f}{\partial \Omega_{j}}}^{+}}
\end{gathered}
$$

O segundo cenário ocorre quando um dos componentes do vetor-gradiente é negativo. O intervalo de soluções para $\frac{\partial f}{\partial \gamma_{j}}<0$ e $\frac{\partial f}{\partial \Omega_{j}}>0$, nas Equações 4.42 e 4.43, é $\frac{\gamma_{j}(t)}{\eta \frac{\partial f}{\partial \gamma_{j}}-}<\lambda<$

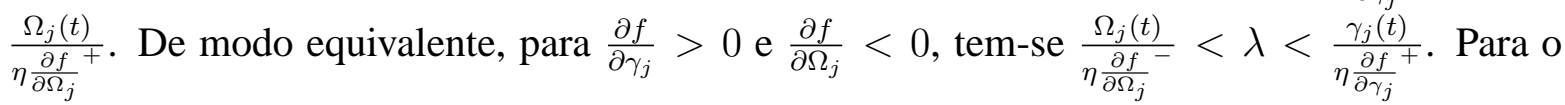
segundo cenário, pode-se escolher $\lambda=\frac{1}{2} \frac{\Omega_{j}(t)}{\eta \frac{\partial f}{\partial \Omega_{j}}}+$ ou $\lambda=\frac{1}{2} \frac{\gamma_{j}(t)}{\eta \frac{\partial f}{\partial \gamma_{j}}}$.

$$
\begin{gathered}
\gamma_{j}(t)-\lambda \cdot \eta{\frac{\partial f}{\partial \gamma_{j}}}^{-}>0 \Rightarrow-\lambda<-\frac{\gamma_{j}(t)}{\eta \frac{\partial f}{\partial \gamma_{j}}-} \Rightarrow \lambda>\frac{\gamma_{j}(t)}{\eta{\frac{\partial f}{\partial \gamma_{j}}}^{-}} \\
\Omega_{j}(t)-\lambda \cdot \eta{\frac{\partial f}{\partial \Omega_{j}}}^{+}>0 \Rightarrow-\lambda>-\frac{\Omega_{j}(t)}{\eta{\frac{\partial f}{\partial \Omega_{j}}}^{+}} \Rightarrow \lambda<\frac{\Omega_{j}(t)}{\eta{\frac{\partial f}{\partial \Omega_{j}}}^{+}}
\end{gathered}
$$

No terceiro cenário, em que $\frac{\partial f}{\partial \Omega_{j}}<0$ e $\frac{\partial f}{\partial \gamma_{j}}<0$, obtêm-se as Equações $4.44 \mathrm{e} 4.45$, que demonstram que $\lambda$ deve ser menor que $\max \left\{\frac{\gamma_{j}(t)}{\eta \partial \gamma_{j}^{-}}, \frac{\Omega_{j}(t)}{\eta \partial \Omega_{j}^{-}}\right\}$. Observa-se que, para esse caso, é possível definir $\lambda=1$ para manter o valor atual de $\eta$.

$$
\begin{gathered}
\gamma_{j}(t)-\lambda \cdot \eta \frac{\partial f}{\partial \gamma_{j}}->0 \Rightarrow-\lambda<-\frac{\gamma_{j}(t)}{\eta \frac{\partial f}{\partial \gamma_{j}}-} \Rightarrow \lambda>\frac{\gamma_{j}(t)}{\eta \frac{\partial f}{\partial \gamma_{j}}}- \\
\Omega_{j}(t)-\lambda \cdot \eta{\frac{\partial f}{\partial \Omega_{j}}}^{-}>0 \Rightarrow-\lambda<-\frac{\Omega_{j}(t)}{\eta \frac{\partial f}{\partial \Omega_{j}}-} \Rightarrow \lambda>\frac{\Omega_{j}(t)}{\eta{\frac{\partial f}{\partial \Omega_{j}}}^{-}}
\end{gathered}
$$




\subsubsection{Discussão dos resultados obtidos a partir da primeira abordagem}

A implementação da primeira abordagem para adaptação do viés de parâmetros foi realizada, porém, seu funcionamento não refletiu as expectativas e o objetivo desejados. Esse fato é atribuído à grande dependência por parte da técnica de adaptação em relação ao funcionamento do algoritmo de agrupamento. Tradicionalmente, técnicas de otimização baseadas em gradiente exigem um espaço contínuo e diferenciável para sua aplicação. Como esse espaço, no caso desta abordagem, é desconhecido, teve-se que assumir condições, na forma de propriedades de convergência, para obter uma aproximação do vetor-gradiente. Contudo, por meio de experimentos, verificou-se que as condições assumidas para a convergência do aprendizado da arquitetura SONDE, utilizadas na obtenção do vetor-gradiente, raramente são atingidas.

Mesmo quando tais condições são satisfeitas, as suposições e simplificações realizadas demonstraram-se ser inadequadas fazendo com que as adaptações nos valores dos parâmetros não fossem coerentes com as expectativas quanto à obtenção de agrupamentos ao longo do tempo. Essas inadequações resultaram em um pior desempenho da SoNDE ao utilizar adaptação, em termos de representatividade do fluxo de dados. Também observou-se, contra as expectativas do projeto de uma técnica de adaptação guiada pela medição de variações comportamentais presentes em dados, uma grande dependência dos valores iniciais para os parâmetros $\gamma, \Omega$ e, também, $\eta$, o qual se refere à taxa de aprendizado do processo de adaptação. Essa característica indica uma baixa qualidade da técnica de adaptação do viés indutivo.

Ao considerar as propriedades definidas no Capítulo 2, pode-se observar que, devido às características da arquitetura SONDE, a primeira abordagem respeita as propriedades de Completude em Fluxos de Dados (Propriedade 5 e Coerência (Propriedade 7 porém não respeita as propriedades de Invariância Espaço-temporal (Propriedade 4) e Consistência (Propriedade 6).

\subsection{Segunda abordagem para a adaptação do viés indutivo}

\subsubsection{Descrição da segunda abordagem}

A primeira abordagem para a adaptação do viés indutivo apresentou limitações na caracterização das relações entre os parâmetros do algoritmo de agrupamento e a variação de comportamento do fluxo de dados devido à dificuldade de obter propriedades de convergência realistas. Esta segunda abordagem, motivada pelas limitações apresentadas pela primeira, visou prover uma abordagem de adaptação de parâmetros menos dependente das características do algoritmo de agrupamento. A abordagem desenvolvida nessa segunda proposta, 
denominada Data Stream Dynamic Clustering (DSDC), consiste na reunião de técnicas para a detecção de variação de comportamento e adaptação de agrupamentos.

A abordagem DSDC atua por meio de uma estratégia composta de três partes. A primeira parte consiste em definir uma partição de grupos utilizando um algoritmo de agrupamento para conjuntos tradicionais de dados, tal como o K-médias (Hartigan \& Wong, 1979). A segunda parte consiste em descrever as relações entre os estados de comportamento ao longo do tempo. Um estado de comportamento é uma conjunção notável das variáveis de um sistema. Na segunda parte, as relações de transição entre estados são descritas por meio do modelo estatístico de cadeias de Markov (Ciuperca \& Girardin, 2007). Cadeias de Markov representam uma ampla gama de fenômenos com dependências temporais descritas por transições probabilísticas entre estados. Elas são destinadas a representar sistemas em que a probabilidade de transição para um próximo estado $X(t+1)$ depende apenas do estado atual $X(t)$, e não dos anteriores $\{X(t-k), k \geq 1\}$. Nesse cenário, protótipos obtidos a partir do algoritmo K-médias representam os estados de comportamento do sistema-alvo ${ }^{5}$. A terceira parte consiste na utilização de um teste de variação de comportamento do sistema para ativar um método de busca de parâmetros com o objetivo de adaptar o viés indutivo do algoritmo de agrupamento.

A abordagem DSDC conecta as duas primeiras partes de sua estratégia ao mapear os protótipos fornecidos pelo K-médias em estados de cadeias de Markov, conforme ilustrado na Figura 4.5. Nessa figura, três grupos de padrões de entrada que compõem uma partição $\Gamma$ (Gama) são representados pelas letras A, B e C. Cada um dos conjuntos de dados são mapeados para a cadeia de Markov na parte direita da mesma figura. As probabilidades de transição, representadas por pesos nas arestas, são estimadas de acordo com a ordem que os dados são atribuídos, ao longo do tempo, a seus respectivos grupos utilizando um método de estimação de cadeias de Markov baseado em uma amostra longa (Ciuperca \& Girardin, 2007).
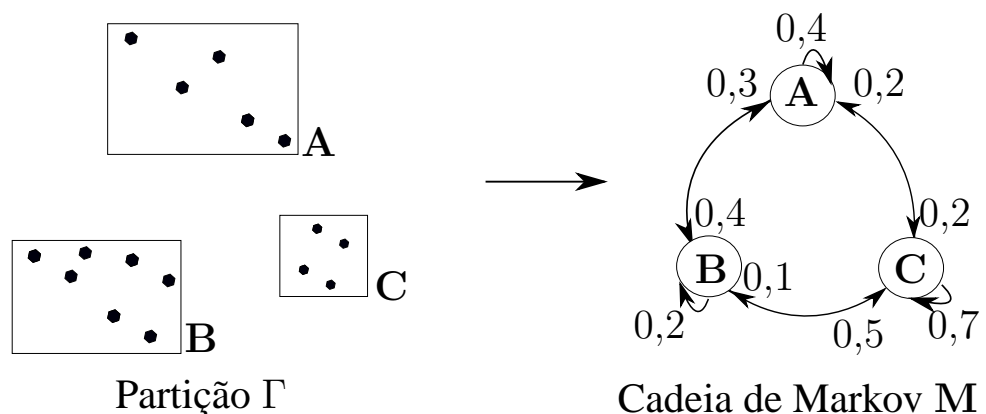

Cadeia de Markov $\mathbf{M}$

Figura 4.5: Ilustração do mapeamento de uma partição de agrupamento em estados de uma cadeia de Markov

O método de estimação das probabilidades de transição de uma cadeia de Markov

\footnotetext{
${ }^{5}$ Sistema é um termo usado para a entidade a partir da qual se obtém os fluxos de dados.
} 
inicia-se com o acúmulo de padrões de entrada. A quantidade necessária de amostras para estimar as probabilidades de transição de uma cadeia com $k$ estados deve depender do erro máximo desejado $\delta$ e de um nível de confiança $\xi$ para as estimativas. Infelizmente, desconhece-se um método para determinar tal quantidade para cadeias de Markov.

No entanto, um método para obter o tamanho do conjunto amostral para estimar as probabilidades de uma distribuição Multinomial pode ser utilizado na forma de uma aproximação (Thompson, 1987). A distribuição Multinomial pode ser considerada como um caso simplificado de cadeias de Markov. Essa distribuição apresenta categorias, diretamente relacionadas aos estados de uma cadeia de Markov cujas probabilidades de transição são tais que $p_{i, j}=p_{k, j}, \forall i, k$. Observando essa similaridade, o método de estimação do tamanho do conjunto amostral para a distribuição Multinomial provê um limitante superior conservador para o número mínimo de padrões de entrada necessário para garantir um erro máximo na estimação das probabilidades de transição da cadeia de Markov. Esse limitante superior (Thompson, 1987, Tabela 1) relaciona a quantidade necessária de amostras para estimar as probabilidades da distribuição Multinomial, o erro máximo $\delta$ e o nível de significância $\xi$ para garantir que esse erro máximo é respeitado.

Considerando essas condições, é possível escolher, para um erro máximo e um nível de significância, o número de padrões a ser acumulado. Para o algoritmo DsDC, escolheu-se como erro máximo de $5 \%$, definido por $\delta=0,05$ e nível de significância $\xi=0,1$, o que resulta em $403 \cdot k$ padrões de entrada necessários para estimação das probabilidades de transição. Esse método, utilizado para determinar o número necessário de padrões para as etapas de agrupamento e estimação de cadeia de Markov, é representado no Algoritmo 1 pela função $\operatorname{tamanho\_ amostral}(k, \delta=0,05, \xi=0,1)$.

Uma vez que um bloco de padrões de entrada é coletado, a execução do algoritmo K-médias provê ao método de estimação de probabilidades de transição de cadeias de Markov, descrito na Seção 4.5, uma partição dos dados (Ciuperca \& Girardin, 2007). Uma matriz $\mathbf{M}$ com $k \times k$ dimensões representa essas probabilidades de transição. Adicionalmente, no processo de estimação de cadeias de Markov, cada transição entre estados é associada a uma probabilidade mínima, tipicamente $10^{-10}$, mesmo se tal transição não foi observada, permitindo a modelagem de sequências de eventos improváveis (Seneta, 2006). Como a matriz de transição M não apresenta probabilidades nulas, um resultado de Álgebra Linear conhecido como Teorema de Perron-Frobenius (Seneta, 2006) garante a existência de pelo menos um autovetor de $\mathbf{M}$, que representa as probabilidades do comportamento assintótico da cadeia de Markov.

As probabilidades de comportamento assintótico representam as tendências a longo prazo do fenômeno, caso esse não varie de comportamento (Seneta, 2006). A obtenção dessas probabilidades é conduzida de acordo com a Equação 4.46, em que $\mathrm{M}$ é a matriz de probabilidades de transições não-nulas e $p$ é o autovetor que representa as probabilidades 
de comportamento assintótico de $\mathrm{M}$ (Seneta, 2006). O autovetor de probabilidades $p$ pode ser estimado, por exemplo, multiplicando $\mathbf{M}$ por um número $t$ suficientemente alto, tal que $\lim _{t \rightarrow \infty} \mathbf{M}^{t}$, até que todas as linhas tenham se igualado a $p$ (Seneta, 2006).

$$
p=\mathbf{M} p
$$

Com o intuito de detectar variações de comportamento no fenômeno observado, cada cadeia de Markov obtida é comparada à anterior por meio de suas probabilidades de comportamento assintótico. Essa comparação permite detectar tais variações, empregando, como medida, a entropia de Shannon (1948) $\mathbb{H}$, definida na Equação 4.47, em que $p_{i}$ representa a probabilidade do sistema assintoticamente convergir ao estado $i$ e $k$ é o número de estados da cadeia de Markov. A entropia de Shannon é isomórfica, ou seja, invariante à representação de cadeias de Markov (Ornstein, 1989). Assim, se os comportamentos assintóticos de duas cadeias de Markov têm a mesma entropia, então elas têm estruturas equivalentes, diferindo apenas na sua representação, i.e., nos protótipos de grupo.

$$
\mathbb{H}(\mathbf{M})=-\sum_{i=1}^{k} p_{i} \log _{2}\left(p_{i}\right)
$$

Para exemplificar a avaliação das variações de comportamento, considere duas cadeias de Markov $\mathbf{M}(0)$ e $\mathbf{M}(1)$ obtidas, respectivamente, em instantes de tempo 0 e 1 . Para verificar variações comportamentais entre elas, calcula-se a entropia do comportamento assintótico de transição entre estados $\mathbb{H}(\mathbf{M}(0))$ e $\mathbb{H}(\mathbf{M}(1))$. Caso a diferença $\frac{|\mathbb{H}(\mathbf{M}(0))-\mathbb{H}(\mathbf{M}(1))|}{\max \{\mathbb{H}(\mathbf{M}(0)), \mathbb{H}(\mathbf{M}(1))\}}$ seja superior a um limiar (próximo a zero) $\tilde{\mathbb{H}}$, sabe-se, então, que ocorreu uma variação de comportamento.

A detecção de variações de comportamento desencadeia o processo de adaptação do algoritmo de agrupamento que, em termos de K-médias, leva a uma busca por uma partição que melhor represente o comportamento atual do fenômeno. No longo prazo, DSDC deverá produzir uma sequência de partições que representa a evolução de um fluxo de dados.

\subsubsection{Algoritmo}

O Algoritmo 1 descreve a implementação da abordagem Data Stream Dynamic Clustering. Esse algoritmo requer a definição de uma constante $\tilde{\mathbb{H}}$, usada para comparar o comportamento representado por duas cadeias de Markov consecutivas, e de um número máximo de grupos utilizado na etapa inicial do algoritmo. DSDC produz uma sequência de partições $\Gamma(t)$, as quais são constituídas a partir do bloco atual $t$ de amostras $b(t)$ obtidas a partir do fluxo de dados. 
O fluxo de dados é divido em blocos a fim de selecionar uma quantidade de padrões de entrada para a aplicação do algoritmo de agrupamento e para a estimativa de cadeias de Markov. A Figura 4.6 apresenta um exemplo em que duas subsequências consecutivas de amostras $b(t-1)$ e $b(t)$ são usadas para estimar as partições $\Gamma(t-1)$ e $\Gamma(t)$ respectivamente, assim como as cadeias de Markov $\mathbf{M}(t-1)$ e $\mathbf{M}(t)$.
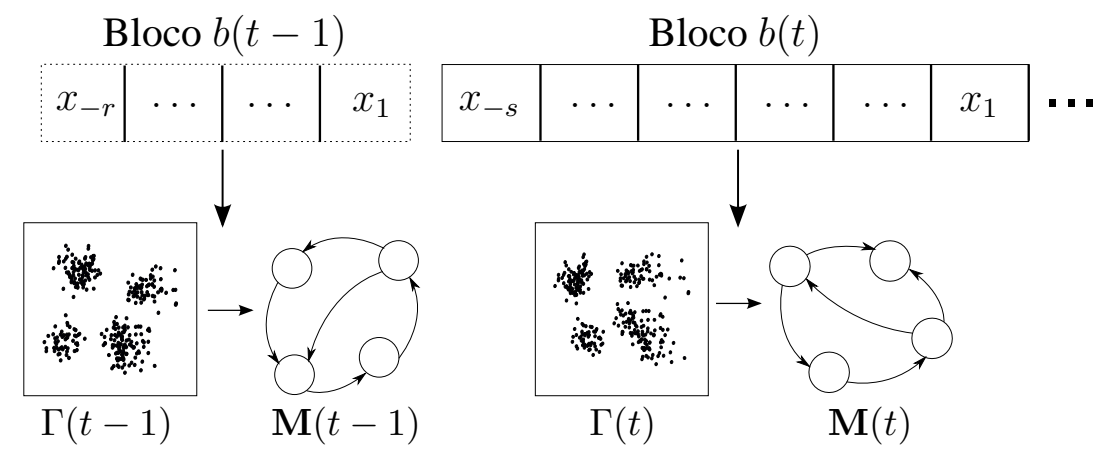

Figura 4.6: Ilustração da abordagem DsDC: coleta e processamento de dados

Antes de cada iteração do laço principal (Linha 3), o número de amostras, neste caso $r$ e $s$, a ser acumulado no bloco de dados seguinte $b(t)$ é determinado de acordo com o número atual de grupos $k$. Conforme apresentado na seção anterior, o tamanho amostral para estimar as probabilidades de transição de uma cadeia de Markov é determinado em termos de um erro máximo $\delta$ e um nível de confiança $\xi$, representado no algoritmo pela função $\operatorname{tamanho\_ amostral}(k, \delta=0,05, \xi=0,1)=403 \cdot k($ Linha 3$)$.

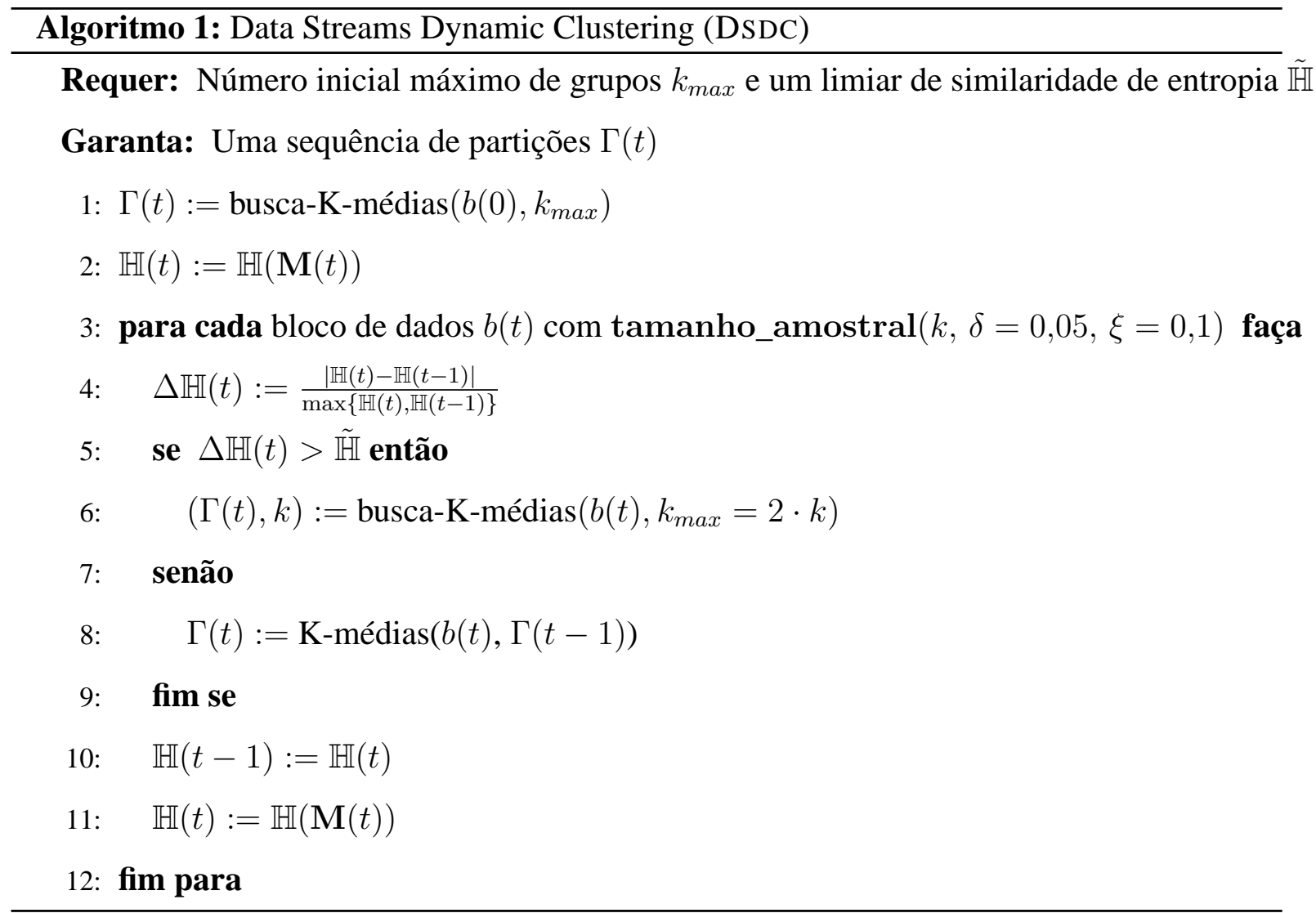

O algoritmo DSDC inicia a obtenção de $\Gamma(t)$ utilizando a primeira parte de amostras 
$b(0)$ (Linha 1). A primeira partição é obtida pelo método busca-K-médias $\left(b(0), k_{\max }\right)$, que executa uma busca exaustiva, utilizando o algoritmo K-médias, para encontrar a partição de $k$ grupos, $\operatorname{com} k$ variando de 2 até o máximo de $k_{\max }$, com o maior valor de Silhueta (Rousseeuw, 1987). Essa medida de qualidade de agrupamentos é definida no intervalo $[-1,1]$, em que quanto maior o valor, maior é a coerência e a separabilidade dos grupos (ver Equação 4.49).

O passo seguinte (Linha 2) consiste em calcular a entropia das probabilidades de comportamento assintótico, que é representado pelo método $\mathbb{H}(t)$, da cadeia de Markov estimada a partir da partição atual $\Gamma(t)$.

No laço principal (Linha 3), primeiramente DSDC verifica se cadeias de Markov consecutivas são isomórficas ao calcular $\Delta \mathbb{H}(t):=\frac{|\mathbb{H}(t)-\mathbb{H}(t-1)|}{\max \{\mathbb{H}(t), \mathbb{H}(t-1)\}}$ (Linha 4), que representa uma proporção da variação de entropia. O valor de $\Delta \mathbb{H}(t)$ é comparado ao limiar $\tilde{\mathbb{H}}$ (Linha 5) para decidir entre a reestruturação dos protótipos e grupos usando, como descrito na Linha 1 , busca-K-médias $\left(b(t), k_{\max }\right)$ (Linha 6 ), ou a sua atualização (Linha 8). A atualização indicada na Linha 8 modifica a partição atual por meio de uma chamada para o algoritmo K-médias na forma $\Gamma(t):=\mathrm{K}$-médias $(b(t), \Gamma(t-1))$. Essa chamada considera os protótipos da partição anterior $\Gamma(t-1)$ para obter uma nova, i.e., $\Gamma(t)$, representando amostras mais recentes. A Linha 6 é utilizada para aumentar o espaço de busca de parâmetros para o dobro do número de grupos atual.

No entanto, se um protótipo em $\Gamma(t-1)$ não for utilizado ou o algoritmo não convergir, DSDC realiza uma nova etapa de pesquisa utilizando o método busca-K-médias.

No final do laço principal, uma nova cadeia de Markov é obtida por meio do método $\mathbb{H}(\mathbf{M}(t))$ (Linha 11), a fim de representar o fluxo de dados.

\subsubsection{Comparação entre algoritmos}

Esta seção compara a abordagem DSDC, a rede neural artificial Grow When Required (GWR (Marsland, 2002) e o algoritmo tradicional K-médias (Hartigan \& Wong, 1979). A GWR foi escolhida devido a sua capacidade de adaptação de agrupamento de acordo com variações de comportamento e, também, devido essa rede ser um bom exemplo dos atuais algoritmos de agrupamento em que fluxos de dados são diretamente processados utilizando aprendizado incremental. O algoritmo K-médias é considerado um padrão de comparação, em virtude da sua relativa simplicidade, uso disseminado e consistência em termos teóricos e experimentais (Jain, 2010). Além disso, o K-médias é frequentemente empregado em algoritmos de agrupamento que primeiro reduzem fluxos de dados a conjuntos de dados finitos e não sequenciais, tais como CLUSTREAM (Aggarwal et al., 2003) e BIRCH (Zhang et al. 1996).

Essa comparação é baseada em fluxos de dados comumente utilizados na literatura, cujas propriedades permitem a observação do desempenho do DSDC sob diversas condições. 
Os fluxos adotados fornecem características de aplicações frequentemente encontradas em contextos reais, tais como alto nível de ruído, caoticidade, não estacionariedade, amostragem de alta frequência e misturas de diferentes fontes (Ishii et al., 2011).

A lista de fluxos de dados selecionados ${ }^{6}$ encontra-se na Tabela 4.1 que mostra o nome de cada fluxo, seu número de dimensões, a natureza (determinística ou estocástica), duração e sua origem (real ou artificial) (Frank \& Asuncion, 2010). Para os experimentos a seguir, cada dimensão de um padrão $\vec{I}(t)$, ou seja, $I_{l}(t)$, é linearmente projetada para intervalo $[0,1]$ na forma $I_{l}(t)=\frac{I_{l}(t)-\min _{t}\left\{I_{l}(t)\right\}}{\max _{t}\left\{I_{l}\right\}-\min _{t}\left\{I_{l}(t)\right\}}$.

Tabela 4.1: Fluxos de dados utilizados na comparação entre DSDC, GwR e K-médias

\begin{tabular}{ccccc}
\hline Fluxo de dados & Dimensões & Natureza & Duração & Origem \\
\hline A & 1 & determinístico & 10093 & artificial \\
B & 3 & estocástico & 34000 & real \\
C & 1 & estocástico & 30000 & real \\
D & 1 & estocástico & 100000 & artificial \\
E & 1 & estocástico & 27204 & real \\
Control Charts & 1 & estocástico & 36000 & artificial \\
Posture & 3 & estocástico & 164860 & real \\
\hline
\end{tabular}

Os fluxos de dados A, B, C, D e E foram utilizados em uma competição de modelagem de séries temporais (Weigend \& Gershenfeld, 1993). O fluxo de dados A é uma sequência obtida a partir de um laser que produz um sinal determinístico e caótico. B é um fluxo de dados multivariado coletado durante o sono de um paciente em laboratório, que contém a frequência cardíaca, o volume do tórax e as medidas de concentração sanguínea de oxigênio. C é um fluxo de dados composto de registros de taxa de câmbio de francos suíços para dólares americanos a partir de 7 de agosto de 1990 a 18 de abril de 1991. D é uma sequência sintética não estacionária baseada no movimento de uma partícula amortecida. O fluxo de dados E consiste em medições astrofísicas ruidosas sobre a curva de luz, com variação temporal da intensidade, da estrela anã branca PG1159-035 durante março de 1989.

O conjunto Control Charts é fornecido como uma coleção de 600 sequências sintéticas não estacionárias de seis tipos diferentes, originalmente destinado a ser utilizado em sistemas de monitoramento (Alcock et al., 1999). A fim de realizar os experimentos, essas sequências foram aleatoriamente ordenadas (sem alterar suas ordens internas), o que resultou em uma sequência com 36000 amostras.

O fluxo de dados Posture foi originalmente coletado para registrar a posição de quatro partes do corpo humano (cintura, tornozelo esquerdo, tornozelo direito e peito), durante várias atividades físicas, a fim de executar o reconhecimento de quedas (Kaluza et al., 2010). Os dados foram sequencialmente coletados a partir de cinco pessoas diferentes. Cada amostra é

\footnotetext{
${ }^{6}$ Esses fluxos de dados estão disponíveis para uso público: http: / / archive. ics . uci .edu/ml/.
} 
descrita por uma posição tridimensional de uma parte do corpo.

As medidas de avaliação de agrupamento Within-Cluster Sum of Squares (WCSS) (Jain, 2010) e Silhueta (Rousseeuw, 1987) foram consideradas para a comparação dos algoritmos. A WCSS (Equação 4.48) é utilizada para avaliar a representatividade dos $k$ protótipos obtidos, em que $\vec{w}_{i}$ é o $i$-ésimo protótipo que representa o grupo $c_{i}$ na partição $\Gamma=$ $\left\{c_{1}, \ldots, c_{k}\right\}$, que é composto de padrões de entrada $\vec{I}_{j} \in c_{i}$. WCSS diminui quando os protótipos melhor representam os dados, ou quando o número de grupos aumenta.

$$
\mathbf{W C S S}=\sum_{i=1}^{k} \sum_{j=1}^{\vec{I}_{j} \in c_{i}}\left\|\vec{I}_{j}-\vec{w}_{i}\right\|^{2}
$$

A medida Silhueta é comumente empregada para avaliar a qualidade de agrupamento com base na dissimilaridade média de amostras dentro do mesmo grupo e na dissimilaridade média entre grupos distintos. A Equação 4.49 define Silhueta, i.e., S, para um conjunto de $k$ elementos, como a média da proporção entre $a_{i}$, i.e., a distância média de cada elemento $i$ para outros elementos de seu grupo, e $b_{i}$, i.e., a distância mínima média do elemento $i$ para elementos de outros grupos. Essa medida é definida para partições com ao menos dois grupos e assume-se -1 para partições com apenas um grupo. A Silhueta não tem uma unidade de medida e varia de -1 , quando os elementos são mal agrupados, até 1 , quando os dados estão perfeitamente agrupados (Rousseeuw, 1987).

$$
\mathbf{S}=\frac{1}{k} \cdot \sum_{i=1}^{k} \frac{b_{i}-a_{i}}{\max \left\{a_{i}, b_{i}\right\}}
$$

\subsubsection{Experimentos}

A fim de realizar experimentos, foi necessário configurar os parâmetros de cada uma das técnicas, ou seja, DSDC, GWR, e K-médias. Para a abordagem DSDC, isso foi realizado a partir de uma análise de sensibilidade de parâmetros (Albertini \& Mello, 2013), que permite observar a importância relativa da atribuição de diferentes valores para cada parâmetro. Dessa forma, o limiar de similaridade de entropia, que é empregado na comparação de similaridade entre cadeias de Markov e para detectar variações de comportamento, foi definido como $\tilde{\mathbb{H}}=$ 0,02 e o número máximo inicial de grupos para a primeira etapa de busca com o algoritmo $\mathrm{K}$-médias foi configurado em $k_{\max }=10$, com o intuito de permitir um espaço razoável de busca pela melhor partição inicial.

Os parâmetros da GWR foram definidos de acordo com valores sugeridos por Marsland
et al. (2002): limite de habituação $h_{T}=0,1$, que é empregado para adaptar grupos de acordo com variações de comportamento; limite de inserção $a_{T}=0,99$, usado para definir 
a pertinência de amostras a grupos; parâmetros de adaptação $\epsilon_{b}=0,2, \epsilon_{n}=0,06, \tau_{b}=3,33$, $\tau_{n}=14,3$, que orientam a aprendizagem de protótipos, e o número máximo de neurônios, que é definido como 40.

A implementacão de K-médias considerada nesta abordagem é a versão de Hartigan \& Wong (1979), disponibilizada pelo R-project (pacote stats) ( $\mathrm{R}$ Development Core Team, 2011). A aplicação do K-médias consistiu no uso do número de grupos encontrado no passo inicial de busca do algoritmo DSDC, o qual seleciona $k$ grupos que provêm a partição com a maior Silhueta encontrada. Com o objetivo de evitar mínimos locais do algoritmo K-médias, partições são obtidas a partir de 30 iterações completas com inicializações aleatórias de protótipos.

Os resultados experimentais estão resumidos na Tabela 4.2. A coluna $k$ lista a média e o desvio padrão do número de grupos e as colunas S e WCSS exibem as médias (e desvio padrão, entre parênteses) de Silhueta e Within-Cluster Sum of Squares para as partições obtidas por um dado algoritmo.

Essas medidas foram calculadas utilizando subsequências de 500 amostras obtidas pela aplicação da técnica de janelas deslizantes (Golab \& Özsu, 2003). Nessa técnica, apenas parte das sequências é utilizada para a obtenção das médias. Por exemplo, a primeira janela deslizante, ou seja, subsequência, é composta por amostras a partir do instante $t=1$ até $t=500$, enquanto que a segunda janela compreende dos valores de $t=2$ até $t=501$ e assim por diante. Cada janela é utilizada para o cálculo das medidas S e WCSS. A sequência de medidas calculadas ao longo do tempo é utilizada para compor suas médias.

Deve-se, porém, tomar em conta que enquanto a avaliação baseada na técnica de janelas deslizantes permite a comparação de diferentes algoritmos, que podem produzir partições em diferentes momentos, algumas subsequências podem ser constituídas por apenas um grupo, fazendo com que a Silhueta seja avaliada como - 1. Pela mesma razão, o uso de janelas deslizantes pode produzir variações no número de grupos $k$ durante a avaliação do algoritmo K-médias, conforme observado na Tabela 4.2.

Para verificar a validade dos resultados, foram realizados testes de hipótese de Wilcoxon-Whitney (Mann \& Whitney, 1947), nos quais o resultado dos algoritmos K-médias e GWR são comparados ao obtidos com o DSDC. As hipóteses nulas $h_{0}$ consideradas foram: a média da Silhueta, ou WCSS, obtida a partir do algoritmo K-médias, ou GWR, é maior que aquela obtida com DSDC. A rejeição de $h_{0}$ é denotada pelo símbolo 'o' quando o $p$-value do teste de hipótese é menor que o nível de significância de 0,05 . De modo similar, o símbolo ' $\bullet$ representa o cenário oposto, em que ocorre a rejeição de $h_{0}$ sendo: a Silhueta média, ou WCSS, obtida com um dado algoritmo é menor que a obtida com o DSDC.

Analisando esses resultados, pôde-se verificar que, em geral, os testes de hipótese sugerem que DSDC apresenta maior desempenho que o algoritmo K-médias, o qual não se 
Tabela 4.2: Resultados para DSDC, GWR e K-médias: média e desvio padrão dos valores para o número de grupos $k$, a Silhueta $\mathbf{S}$ e a WCSS

\begin{tabular}{ccccc}
\hline Fluxo de dados & Técnica & $k$ & S & WCSS \\
\hline $\mathbf{A}$ & DSDC & $2.6(0.92)$ & $0.47(0.29)$ & $37.85(2.12)$ \\
& K-médias & $2.6(0.91)$ & $0.47(0.3)$ & $37.96(2.09) \bullet$ \\
B & GWR & $11.72(0.45) \bullet$ & $0.09(0.08) \circ$ & $16.1(2.88) \circ$ \\
& DSDC & $3.05(1.39)$ & $0.26(0.36)$ & $33.37(14.2)$ \\
& K-médias & $5.49(1.51) \bullet$ & $0.32(0.15) \circ$ & $24.98(8.84) \circ$ \\
$\mathbf{C}$ & GWR & $13.23(4.86) \bullet$ & $0.15(0.13) \circ$ & $21.31(7.26) \circ$ \\
& DSDC & $2.19(0.79)$ & $0.25(0.62)$ & $6.64(2.77)$ \\
& K-médias & $1.98(0.69) \circ$ & $0.16(0.64) \circ$ & $7.85(3.47) \bullet$ \\
D & GWR & $2(0) \circ$ & $0.05(0.04) \circ$ & $12.13(6.36) \bullet$ \\
& DSDC & $2.44(0.84)$ & $0.49(0.23)$ & $42.06(4.18)$ \\
& K-médias & $2.4(0.79) \circ$ & $0.49(0.23)$ & $42.53(3.47) \bullet$ \\
& GWR & $14.31(1.43) \bullet$ & $0.36(0.06) \circ$ & $8.83(2.64) \circ$ \\
& DSDC & $2.07(0.38)$ & $0.53(0.11)$ & $25.71(5.95)$ \\
& K-médias & $2.07(0.38)$ & $0.53(0.11)$ & $25.71(5.95)$ \\
Control Charts & GWR & $3(0) \bullet$ & $-0.05(0.03) \circ$ & $43.11(10.34) \bullet$ \\
& DSDC & $3.85(0.9)$ & $0.51(0.16)$ & $21.26(3.97)$ \\
& K-médias & $3.51(1.06) \circ$ & $0.47(0.21) \circ$ & $25.06(1.92) \bullet$ \\
Posture & GWR & $13.35(2) \bullet$ & $0.35(0.05) \circ$ & $8.41(1.7) \circ$ \\
& DSDC & $4.06(3.06)$ & $0.3(0.35)$ & $65.53(22.04)$ \\
& K-médias & $2.35(0.78) \circ$ & $0.27(0.36) \circ$ & $76.29(17.84) \bullet$ \\
& GWR & $19.83(6.65) \bullet$ & $0.25(0.12) \circ$ & $30.82(9.39) \circ$ \\
\hline
\end{tabular}

O símbolo 'o' denota médias estatisticamente mais baixas que as obtidas com DSDC.

O símbolo ' $\bullet$ ' denota médias estatisticamente mais altas que as obtidas com DSDC.

adapta à variação de comportamento presente em fluxos de dados, e que a rede neural artificial GWR, a qual adapta suas partições ao longo do tempo, porém apresenta viés indutivo estático. Observa-se que a Silhueta média obtida com DSDC para todos fluxos de dados é, pelo menos, similar à obtida pelas outras técnicas. Adicionalmente, em comparação ao K-médias, DSDC apresentou baixos valores de WCSS, embora ambos os algoritmos obtiveram número de grupos $k$ similares. A rede GWR destaca-se por produzir uma média de grupos maior que 10 para 5 dos fluxos de dados, o que acarretou baixos valores de WCSS,

As Figuras 4.7 e 4.8 ilustram duas execuções típicas ${ }^{7}$ dos algoritmos em relação aos fluxos de dados B e Control Charts. A Figura 4.7 apresenta os resultados para o fluxo de dados B, nos quais o algoritmo DSDC exibiu valores instáveis, porém, bons de Silhueta. Apesar de sua instabilidade, a Silhueta obtida com DSDC superou a obtida com os algoritmos K-médias e GWR Por outro lado, a rede GWRé melhor avaliada quando considerando WCSS, ao custo de um alto número de grupos.

Os resultados para o fluxo de dados Control Charts, apresentados na Figura 4.8, ilustra um cenário distinto. A curva de Silhueta para DSDC foi mais estável e com valores mais altos que aquela resultante do K-médias e GWR. Enquanto isso, embora os resultados de

\footnotetext{
${ }^{7}$ Os resultados exibidos nas figuras foram suavizados por meio de curvas de Bézier.
} 


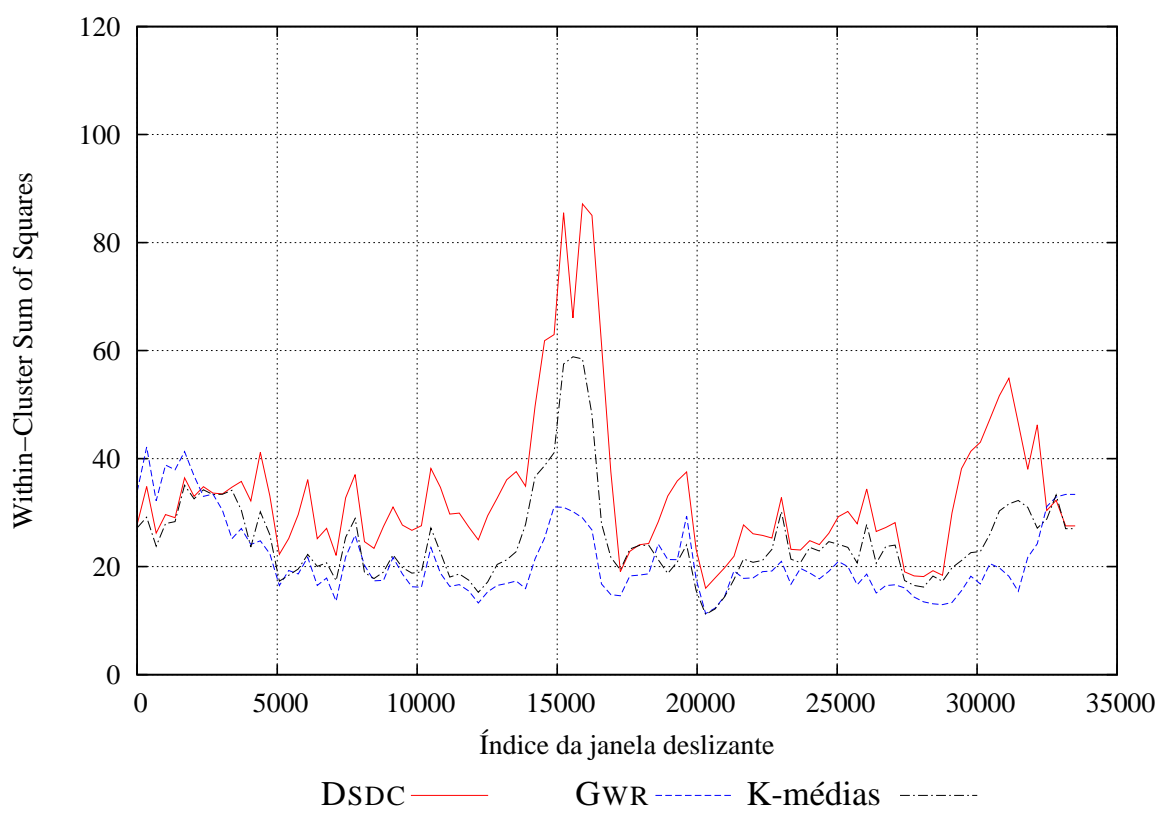

(a) WCSS

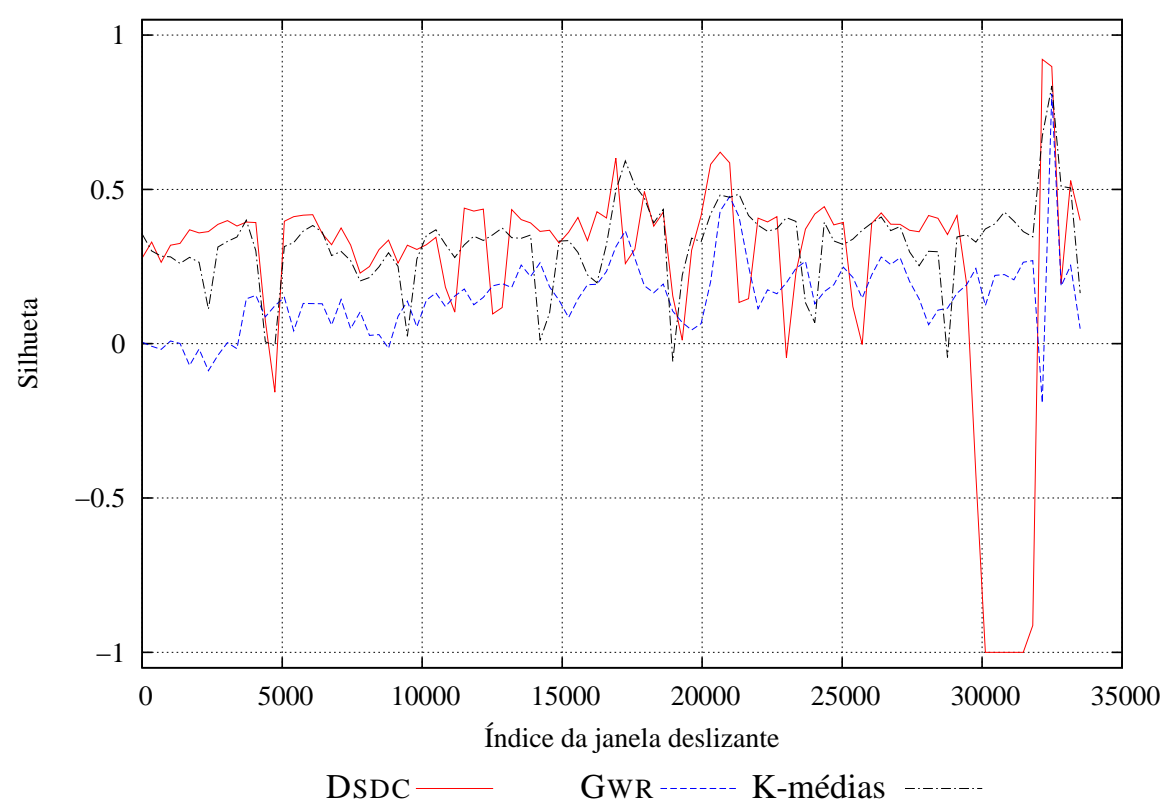

(b) Silhueta

Figura 4.7: Fluxo de dados B: resultados de WCSS e Silhueta 


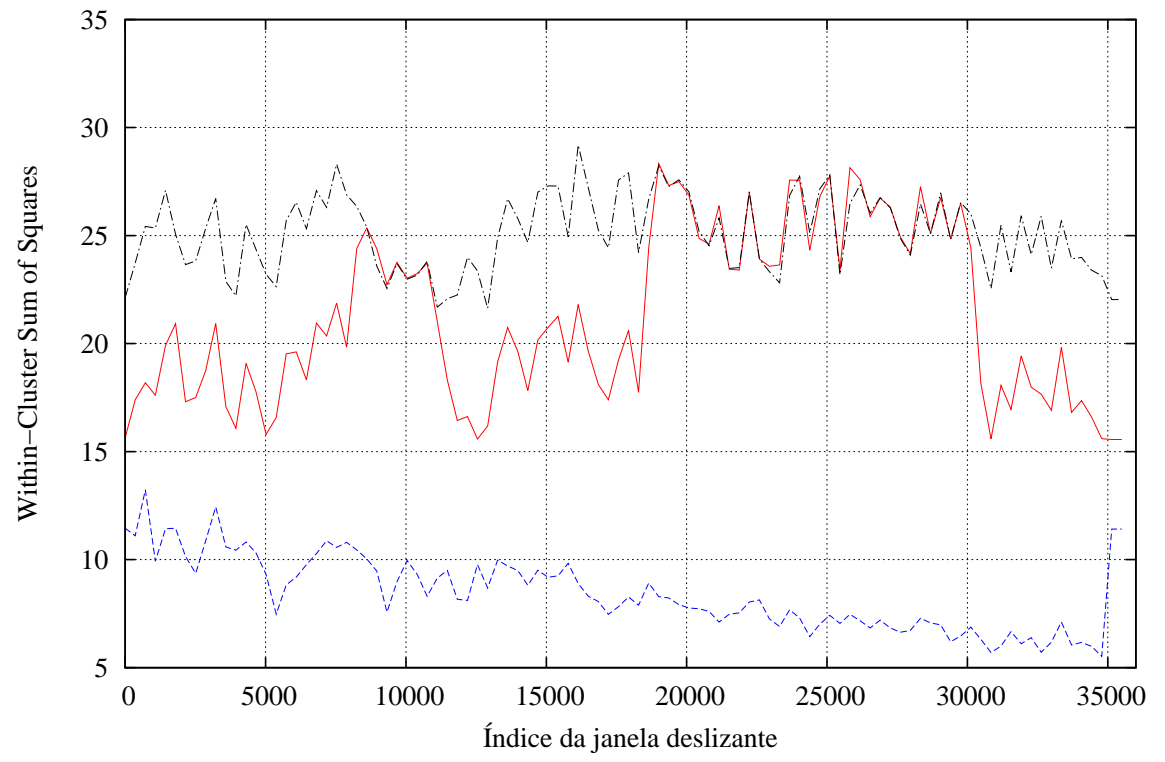

DSDC — GWR --.-- K-médias

(a) WCSS

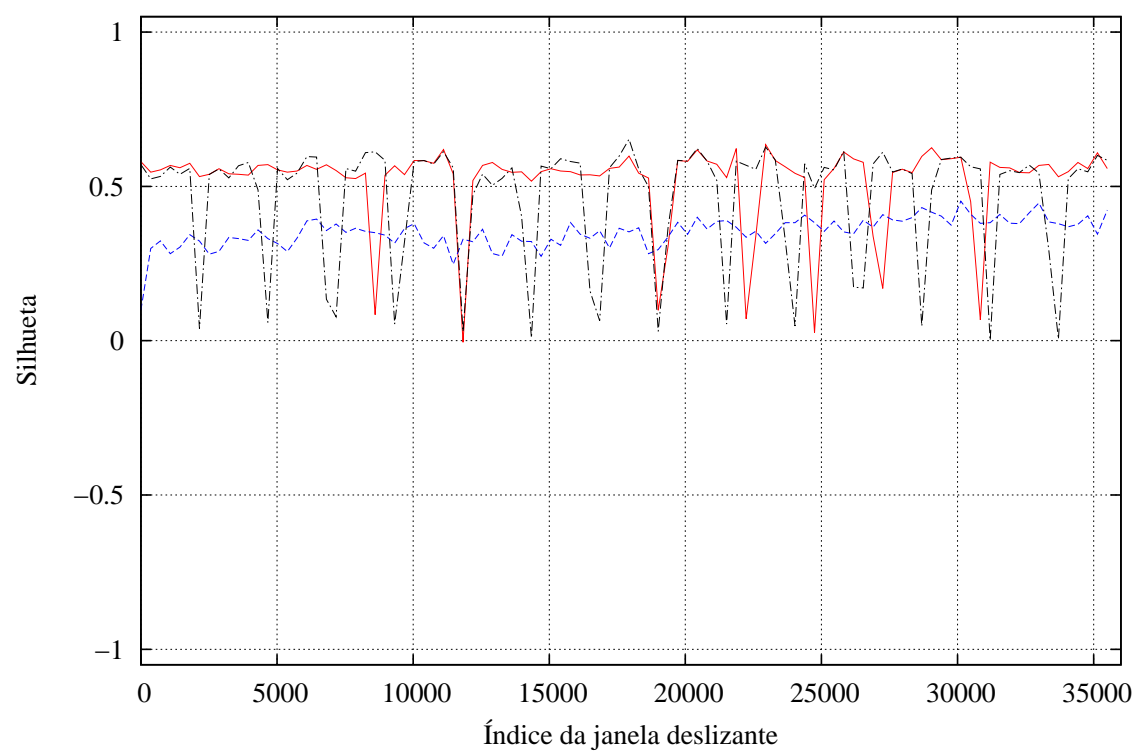

DSDC — GWR ------ K-médias

(b) Silhueta

Figura 4.8: Fluxo de dados Control Charts: resultados de WCSS e Silhueta 
WCSS para o algoritmo K-médias foram mais estáveis, os obtidos com o algoritmo DSDC foram similares ou melhores que os atingidos pelo K-médias, fato atribuído à adaptação do número de grupos $k$ de acordo com a variação de comportamento presente no fluxo de dados. Portanto, esses resultados demonstraram as vantagens da adaptação de viés de parâmetros de agrupamento de fluxos de dados.

\subsubsection{Discussão dos resultados obtidos a partir da segunda abordagem}

A abordagem Data Streams Dynamic Clustering (DSDC) utiliza resultados de Teoria da Informação (Shannon, 1948) e Sistemas Dinâmicos (Ornstein, 1989) para identificar variações de comportamento e atualizar o viés de parâmetros. Experimentos com essa abordagem demonstram resultados interessantes quando aplicados a fluxos de dados provenientes de diferentes campos, com distintas propriedades. Resultados foram comparados com os obtidos por meio do algoritmo K-médias, que não possui adaptação, e da rede neural artificial Grow When Required, que adapta agrupamentos em função de variações de comportamento.

Para a maior parte dos fluxos de dados considerados, DSDC superou GWR K-médias, evidenciando que a adaptação de parâmetros melhora a representatividade dos agrupamentos obtidos. Contudo, a principal desvantagem da DSDC se dá pelo uso de blocos de dados e de um algoritmo de agrupamento tradicional, o que desconsidera a continuidade dos dados. Essa característica limita a compreensão de dinâmicas temporais do fenômeno observado. Além disso, o modo de atualização dos grupos de dados (em blocos) não garante continuidade na produção de partições para fluxos de dados (ver Propriedade da Consistência, Capítulo 2), o que limita a compreensão de dados dessa natureza. Além disso, essa abordagem não respeita as outras propriedades definidas, i.e., Completude em Fluxos de Dados (Propriedade 5, Coerência (Propriedade 7) Invariância Espaço-temporal (Propriedade 4).

\subsection{Terceira abordagem para a adaptação de viés indutivo}

A terceira abordagem desenvolvida nesta tese foi motivada pela necessidade de adaptar o viés indutivo de algoritmos de agrupamento de maneira contínua, provendo informações imediatas relativas a variações de comportamento de fenômenos. Diferentemente de algoritmos de agrupamento que tratam fluxos de dados como conjuntos tradicionais (Zhang et al., 1996; Aggarwal et al., 2003), em que a compreensão do comportamento presente em dados é limitada, algoritmos contínuos permitem avaliar fluxos de dados conforme sua coleta, provendo uma melhor compreensão do comportamento de fenômenos ao longo do tempo.

Essa abordagem foi inspirada pelas propriedades de memória e esquecimento das equações de aprendizado da arquitetura SONDE, definidas nas Equações 4.1 ou 4.2. Tais 


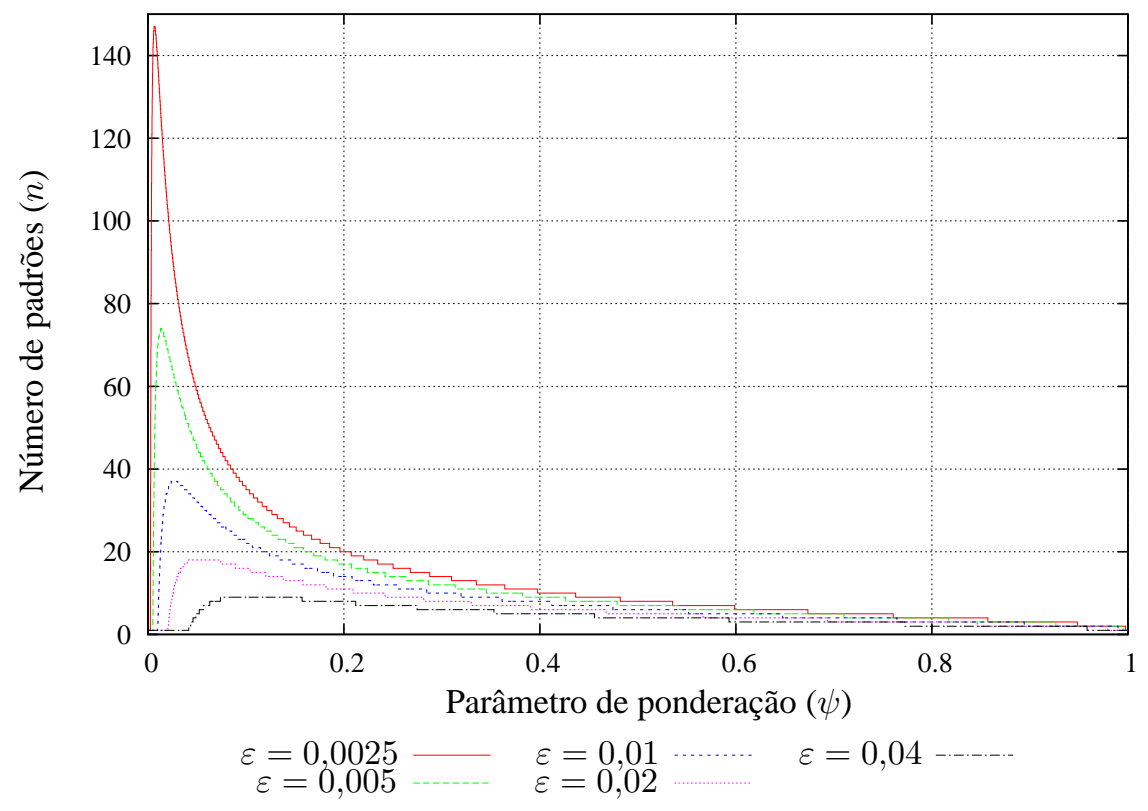

Figura 4.9: Caracterização do número $n$ de padrões necessários para aplicar um fator de esquecimento $\varepsilon \in\{0,0025,0,005,0,01,0,02,0,04\}$ em um padrão passado $\vec{I}(t-n)$, conforme o parâmetro de ponderação $\psi$

equações são médias móveis exponenciais ponderadas (MMEP $)$ no formato da Equação 4.50 , na qual $\vec{m}(t)$ é a média da sequência dos padrões de entrada $\{\ldots, \vec{I}(t-1), \vec{I}(t)\}$, obtida a partir de um parâmetro de ponderação $\psi \in(0,1]$.

$$
\vec{m}(t)=(1-\psi) \cdot \vec{m}(t-1)+\psi \cdot \vec{I}(t)
$$

Com o intuito de compreender a influência do parâmetro de ponderação $\psi$ na composição da memória da equação que define uma MMEP, avaliou-se a taxa de esquecimento incorrida pela adaptação a novos padrões. Essa avaliação foi realizada a fim de estabelecer o número $n$ de padrões de entrada necessários, segundo a desigualdade apresentada na Equação 4.51, para a média móvel exponencial ponderada aplicar um fator de esquecimento $\varepsilon$ a um padrão de entrada aprendido no instante $t-n$ do passado.

$$
\varepsilon \leq \psi \cdot(1-\psi)^{n-1}, n \in \mathbb{Z}, n \geq 1
$$

Uma ilustração do uso dessa desigualdade, para diferentes fatores de esquecimento $\varepsilon$, é apresentada na Figura 4.9. Nela, cada curva traçada caracteriza a menor quantidade $n$ de padrões necessários para a aplicação do fator de esquecimento $\varepsilon$, considerando o parâmetro de ponderação $\psi$. A partir dessa figura é possível observar que, por exemplo, dado um parâmetro de ponderação $\psi=0,2$, o padrão de entrada $\vec{I}(t-20)$ é ponderado por 0,0025 na média $\vec{m}(t)$.

A partir dessa figura, cogita-se que existe um valor adequado para o parâmetro de 
ponderação das equações de aprendizado da arquitetura SONDE proporcional à exigência de manter padrões de entrada em memória, o que, por sua vez, depende da variação de comportamento do fenômeno observado. Dessa forma, conforme o fator de esquecimento $\varepsilon$ aumenta, o valor do parâmetro de ponderação $\psi$ também deve aumentar a fim de permitir a maximização do número de padrões de entrada em memória.

Para melhor compreender esse conceito, considere que um evento extraordinário acontece na vida de uma pessoa, tal como ganhar um alto prêmio na loteria. Nesse momento essa pessoa apresentará um alto fator de esquecimento, e.g., $\varepsilon=0,04$ e, portanto, ela dará pouca importância para os eventos que aconteceram antes disso. Observe na Figura 4.9 que a curva obtida com o maior fator de esquecimento apresentado $\varepsilon=0,04$ apresenta os menores valores para o número de padrões de entrada em memória. Porém, se essa pessoa utilizar um parâmetro de ponderação de cerca de $\psi=0,1$, o número de eventos $n$ em sua memória será maximizado. Por outro lado, se a vida dessa pessoa é monótona, ela dará importância a muitos eventos passados, e.g., $n=140$, com um baixo fator de esquecimento próximo de $\varepsilon=0,0025$, o que indica que o parâmetro de ponderação de sua memória está próximo de $\psi=0,01$.

Dessa forma, no contexto dessa abordagem, fluxos de dados instáveis, que apresentam maior variação de comportamento, requerem fatores de esquecimento mais altos, o que exige a adaptação do parâmetro de ponderação $\psi$, constituinte do viés indutivo da arquitetura SONDE, para valores mais altos. Contudo, esse parâmetro não pode ser adaptado além de certos limites, pois pode ameaçar a representação da tendência atual dos padrões de entrada.

\subsubsection{Descrição da terceira abordagem}

Essa abordagem é baseada na associação entre a variação comportamental presente em fluxos de dados e a definição de fatores de esquecimento. Conforme previamente mencionado, o fator de esquecimento de equações MMEP é proporcional à necessidade de adaptação de acordo com a variação de comportamento presente nos dados. É possível avaliar essa variação utilizando uma medida de estabilidade relacionada às equações MMEP da SONDE, as quais são dependentes dos padrões de entrada coletados.

Para caracterizar a estabilidade de sequência de dados, empregou-se uma medida baseada no cálculo da derivada numérica dos padrões de entrada, a qual avalia o quanto uma função muda de acordo com os dados observados, mesmo na falta de conhecimento analítico sobre tal função. Essa medida pode ser associada à modificação dos parâmetros de ponderação $\gamma$ e $\Omega$, referentes ao parâmetro $\psi$, nas equações de adaptação das estruturas de conhecimento da arquitetura SONDE,

Com o objetivo de definir tal associação, buscou-se por uma função para mapear a derivada numérica dos dados, definida em $\mathbb{R}$, no domínio de $\psi$, ou seja, $(0,1]$. Essa função 
deve fazer com que, quando ocorra completa estabilidade dos dados, a memória da MMEP tenda ao seu máximo, ou seja, $\psi \approx 0$. Por outro lado, quando a instabilidade for alta, o valor do parâmetro $\psi$ é aumentado, provocando, no limite, o esquecimento instantâneo do passado.

Por exemplo, suponha a situação hipotética de dirigir um carro sem freio em um lugar em condições ideais, nas quais uma pista tem subidas e descidas e o objetivo é economizar combustível. Assume-se que a única situação em que se consome combustível é quando se deve subir esse relevo, enquanto que na descida não se recupera energia. Se o carro está em uma superfície plana, basta não usar combustível, pois existe inércia e não há atrito para reduzir sua velocidade. Nessa situação, alterações de relevo exigem soltar ou pressionar o pedal.

No contexto de fluxo de dados, a alteração de relevo representa a variação de comportamento em fluxo de dados e o nível do pedal equivale ao parâmetro de ponderação $\psi \in(0,1]$. Assim, pode-se considerar $\psi$ como um fator que determina o quanto o pedal está pressionado para injetar combustível, sendo que o pedal completamente pressionado corresponde a $\psi=1$ e, solto, a $\psi=0$.

\subsubsection{Formulação}

A estimação de uma MMEP $\vec{m}(t)$ (Equação 4.50) para um fluxo de dados com variação de comportamento deve considerar a adaptação de seu parâmetro de ponderação $\psi$, de forma que $\vec{m}(t)$ represente adequadamente o fenômeno observado.

A adaptação do parâmetro $\psi(t)$ deve mapear a avaliação de estabilidade no intervalo $[0,1]$ e, em seguida, utilizar esse valor para adaptar $\psi(t)$ em sua direção. No contexto desta abordagem, define-se como medida de estabilidade dos padrões de entrada o valor absoluto da derivada numérica dos padrões de entrada, i.e., $\left|\frac{d \vec{I}(t)}{d t}\right|$.

Para mapear, da maneira descrita na seção anterior, a medida de estabilidade $\left|\frac{d \vec{I}(t)}{d t}\right|$, que é definida em $\mathbb{R}$, para o intervalo $[0,1]$, definiu-se uma função não linear $T(\cdot)$ com as seguintes propriedades $T(0)=0$ e $\lim _{x \rightarrow \infty} T(x)=1$. A função escolhida para $T(\cdot)$, que apresenta tais propriedades, foi $T(x)=\tanh (x)$.

A adaptação de $\psi(t)$ deve ser realizada utilizando a função $T\left(\left|\frac{d \vec{I}(t)}{d t}\right|\right)$ para manter o valor de $\psi(t)$, caso $\left|\frac{d \vec{I}(t)}{d t}\right|$ não tenha se modificado significantemente, e altere $\psi(t)$, caso as instabilidades observadas nos dados forem de magnitude suficientemente alta.

A função de adaptação $F(x, y)$, definida em termos da função de mapeamento $T(\cdot)$, deve ser uma função monotônica crescente em $x$ (Equação4.52), ou seja, $F\left(x_{1}, y\right)<F\left(x_{2}, y\right)$ se, e somente se, $x_{1}<x_{2}$, em que $y$ é um valor de referência. Dessa forma a adaptação é realizada com magnitude $F\left(T\left(\left|\frac{d \vec{I}(t)}{d t}\right|\right), \psi(t)\right)$. 


$$
F(x, y)=f(x-y+g(y))
$$

Além disso, a função $F(x, y)$ deve ser tal que $F(x, y)=y$ se, e somente se, $x=y$ e $F(x, y)<y$ se, e somente se, $x<y$, em que $g(y)$ é a função inversa de $f(x)$. Uma escolha natural para $f(x)$ é $f(x)=\frac{\tanh (x)+1}{2}$ e, $\operatorname{assim}, g(y)=\operatorname{arctanh}(2 \cdot(y-0,5))$.

O Algoritmo 2 apresenta as regras para adaptação de uma MMEP para $\vec{m}(t)$ e de seu parâmetro de ponderação $\psi(t)$ de acordo com um fluxo de dados $\vec{I}(t)$.

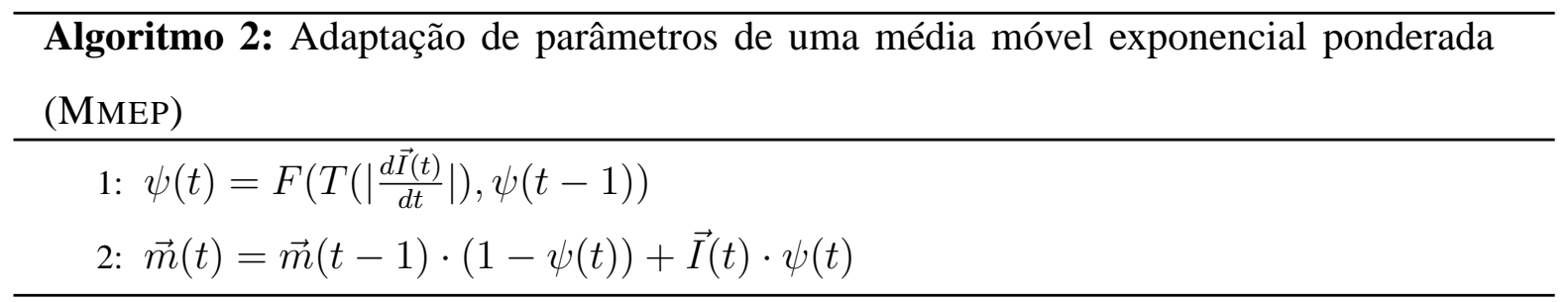

\subsubsection{Experimentos}

Segundo a hipótese definida nesta tese de doutorado, caso o comportamento em um fluxo de dados varie, o viés indutivo de parâmetros do algoritmo de agrupamento deve ser adaptado. Os seguintes experimentos têm o intuito de apresentar cenários que demonstram o funcionamento da abordagem proposta e confirmam tal hipótese ${ }^{8}$.

No contexto dos experimentos a seguir, assuma que uma média MMEP $\vec{m}(t)$ é um estimador para o modelo ideal do fenômeno observado. Portanto, o grau de representatividade dessa média é proporcional a sua distância ao estado verdadeiro. Esse grau de representatividade é obtido a partir da aplicação de uma técnica para o cálculo de distâncias entre sequências denominada Dynamic Time Warping (DTW) (Tormene et al., 2009; Giorgino, 2009).

A distância DTW é tolerante a eventuais desalinhamentos temporais entre sequências, o que é importante para levar em conta possíveis atrasos de aprendizado na avaliação do grau de representatividade. Por exemplo, quando se compara o modo de andar entre duas pessoas, embora elas possam apresentar diferentes velocidades ao longo do tempo, é possível que o comportamento seja similar, o que é mensurado pela distância DTw. Dessa forma, tal distância provê a similaridade de comportamento entre médias MMEPe modelos ideais, o que não seria obtido, por exemplo, a partir do emprego de uma distância euclidiana.

Os experimentos foram realizados a partir da geração de sequências sintéticas de dados com ruídos. Cada sequência sintética é composta de um componente determinístico, que representa o modelo ideal para o fenômeno observado, e um componente com ruídos, que representa interferências indesejadas no processo de coleta dos dados (Ishii et al., 2011). Por

\footnotetext{
${ }^{8}$ Esses experimentos foram prototificados e executados no ambiente de programacão $R$-proiect (R Development Core Team, 2011).
} 
exemplo, para obter a sequência senoide apresentada na Figura 4.10, executou-se, na linguagem $\mathrm{R}$, o comando $\sin (\operatorname{seq}(0,20$, length $=400))+$ rnorm $(400)$, que utiliza a função seno como o modelo ideal para o fenômeno em estudo e a distribuição de probabilidades Normal $N\left(\mu=0, \sigma^{2}=1\right)$ para representar os ruídos identicamente distribuídos e independentes da sequência.

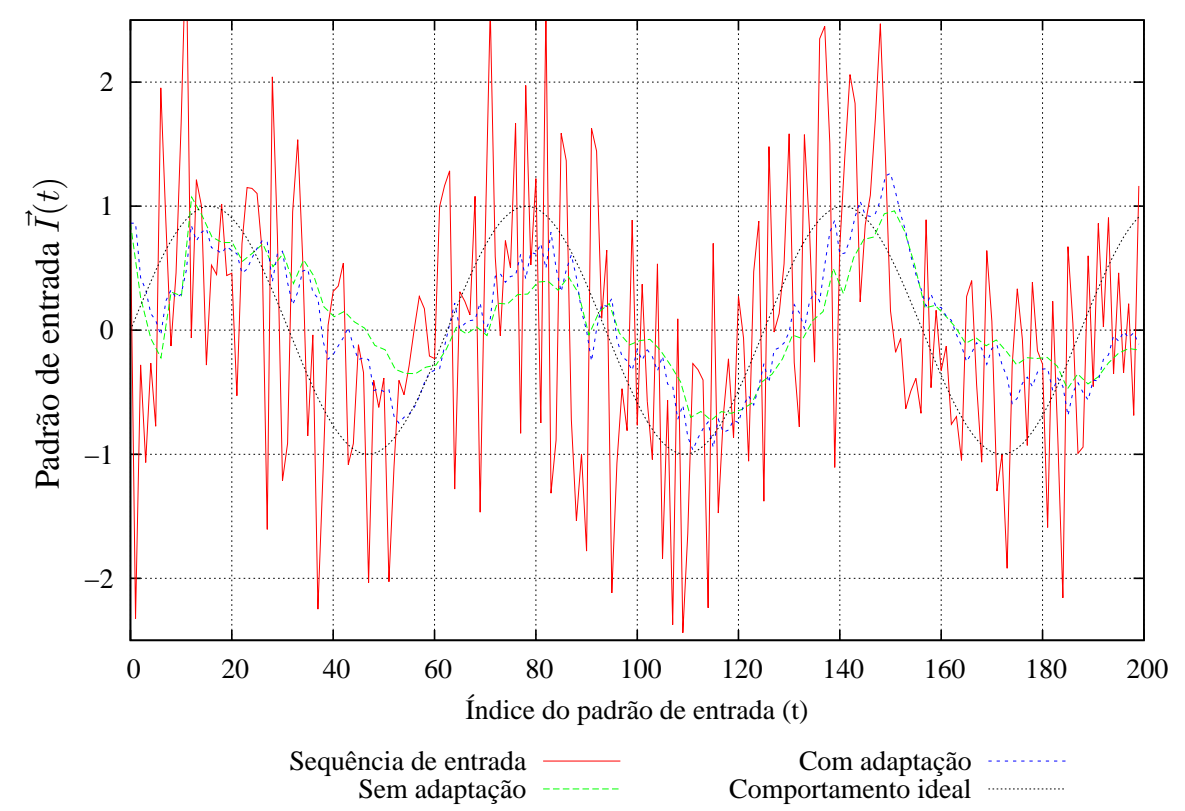

Figura 4.10: Aproximações para uma sequência senoide com ruído Normal $N\left(\mu=0, \sigma^{2}=1\right)$. O melhor parâmetro de ponderação inicial $\psi$ para MMEP, sem a técnica de adaptação atuante, encontrado foi $\psi=0,137$ (distância $\mathrm{DTW}=0,089$ ) e o melhor parâmetro de ponderação inicial para a MMEP, com a técnica de adaptação atuante, foi $\psi=0,186$ (distância DTW = $0,121)$

Na Figura 4.10, apresentam-se curvas de MMEP obtidas a partir da execução da técnica proposta para a adaptação do parâmetro $\gamma$ da arquitetura SONDE e resultados para quando tal técnica não está atuante ao longo do processamento da senoide ruidosa. Os valores iniciais estabelecidos para o parâmetro $\gamma$ da arquitetura SONDE, para ambos cenários, com ou sem a técnica de adaptação atuante, foram obtidos a partir do método buscaParametro(). Esse método, apresentado no Algoritmo 3, emprega a função de otimização de propósito geral disponível no ambiente R optimize() com o intuito de minimizar a distância DTW entre os protótipos de neurônios e o modelo ideal, com base em um fluxo de dados, uma sequência de dados representando o modelo ideal do fenômeno em estudo, e em valores constantes para os 
parâmetros $\Omega$ e $\alpha_{0}$.

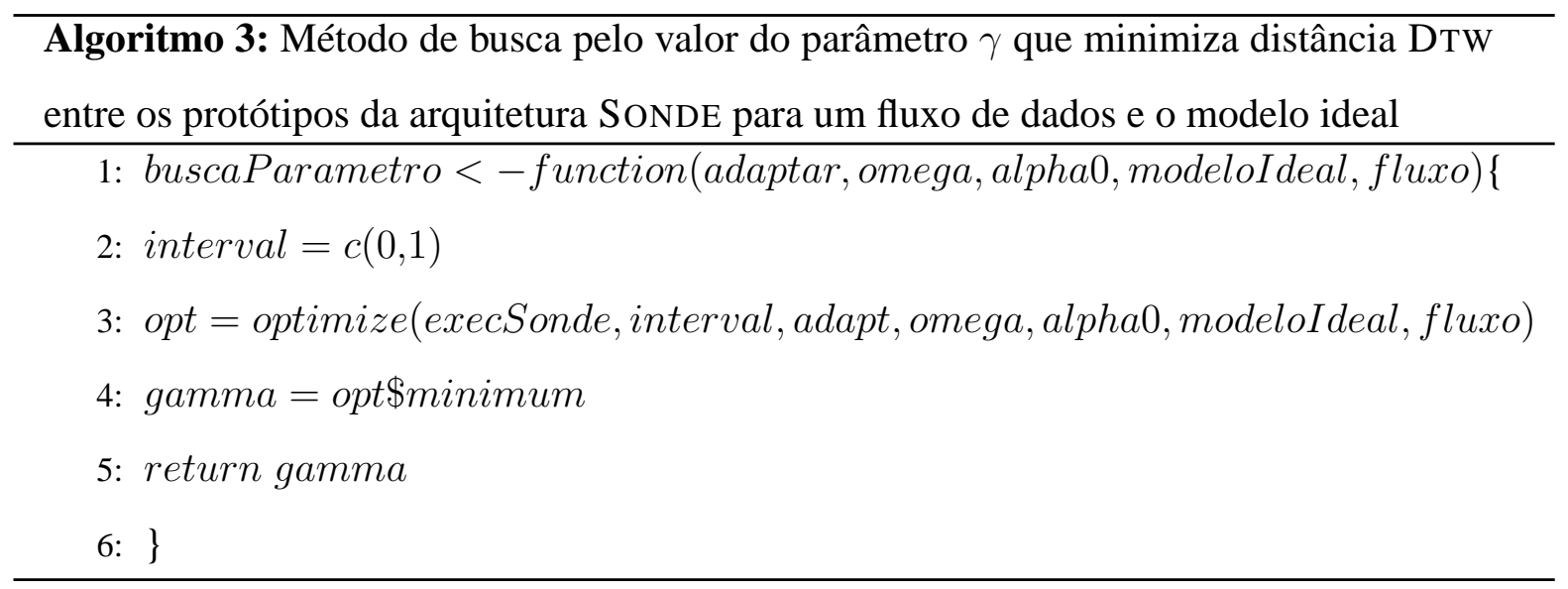

O método buscaParametro() encontra, dessa forma, o melhor valor inicial para o parâmetro de ponderação $\gamma$, ou seja, aquele que provê a menor distância DTW possível, a partir da execução de um método denominado execSonde (Algoritmo 4), cujo objetivo é executar uma instância da SONDE e obter a distância DTw em relação ao modelo ideal. Esse tipo de procedimento para definir o viés indutivo de parâmetros é comumente utilizado na etapa de projeto de algoritmos de agrupamento.

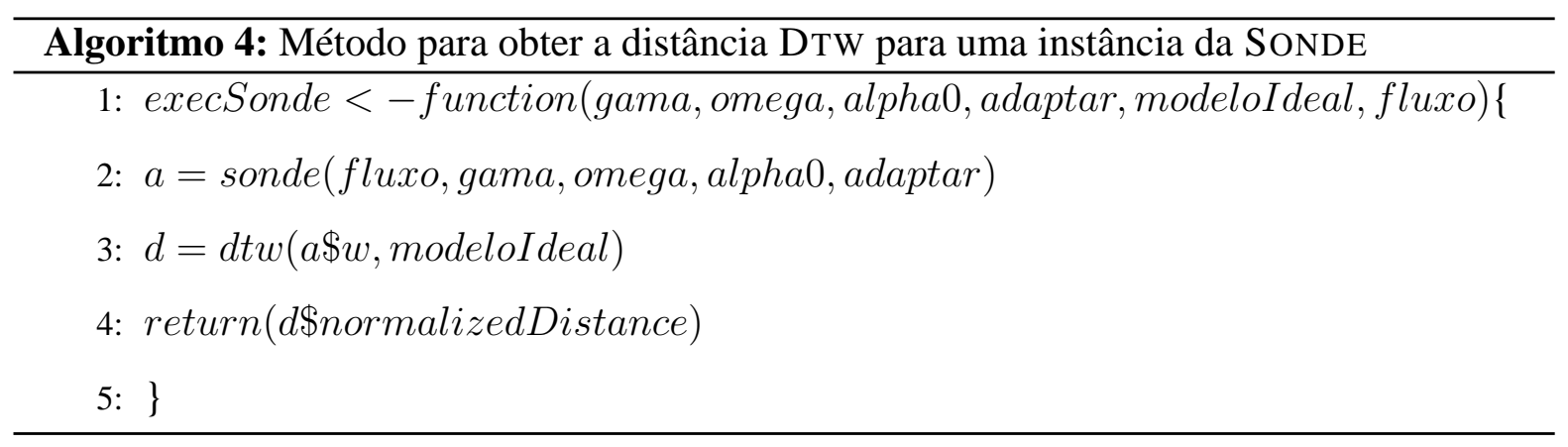

A princípio, ao observar os resultados na Figura 4.10 obtidos a partir do procedimento previamente descrito, pode-se imaginar que a técnica de adaptação não é necessária, pois os resultados obtidos com a técnica de adaptação atuante e não atuante são similares.

Entretanto, observe, na Figura 4.11, as curvas de distâncias DTW entre o modelo ideal, i.e., a função seno, e o modelo obtido a partir do processamento da senoide ruidosa pela arquitetura SONDE considerando a variação de seu parâmetro de ponderação $\gamma$ (eixo horizontal da Figura 4.11) e os parâmetros mantidos constantes $\Omega=0$ e $\alpha_{0}=0,1$. Nessa figura, apresenta-se uma curva de distâncias DTw obtida com a técnica de adaptação atuante, i.e., curva com adaptação, uma curva obtida sem a utilização da técnica de adaptação, i.e., curva sem adaptação e, para fins de referência, a linha de comparação com a senoide ruidosa, que representa a distância entre o modelo ideal e a sequência ruidosa coletada. Essas curvas foram obtidas a partir da variação do parâmetro inicial $\gamma$, em todo seu intervalo $(0,1]$, com passos de variação iguais a 0,001 .

A partir das curvas na Figura 4.11 pode-se observar que caso $\gamma$ seja inicializado com 


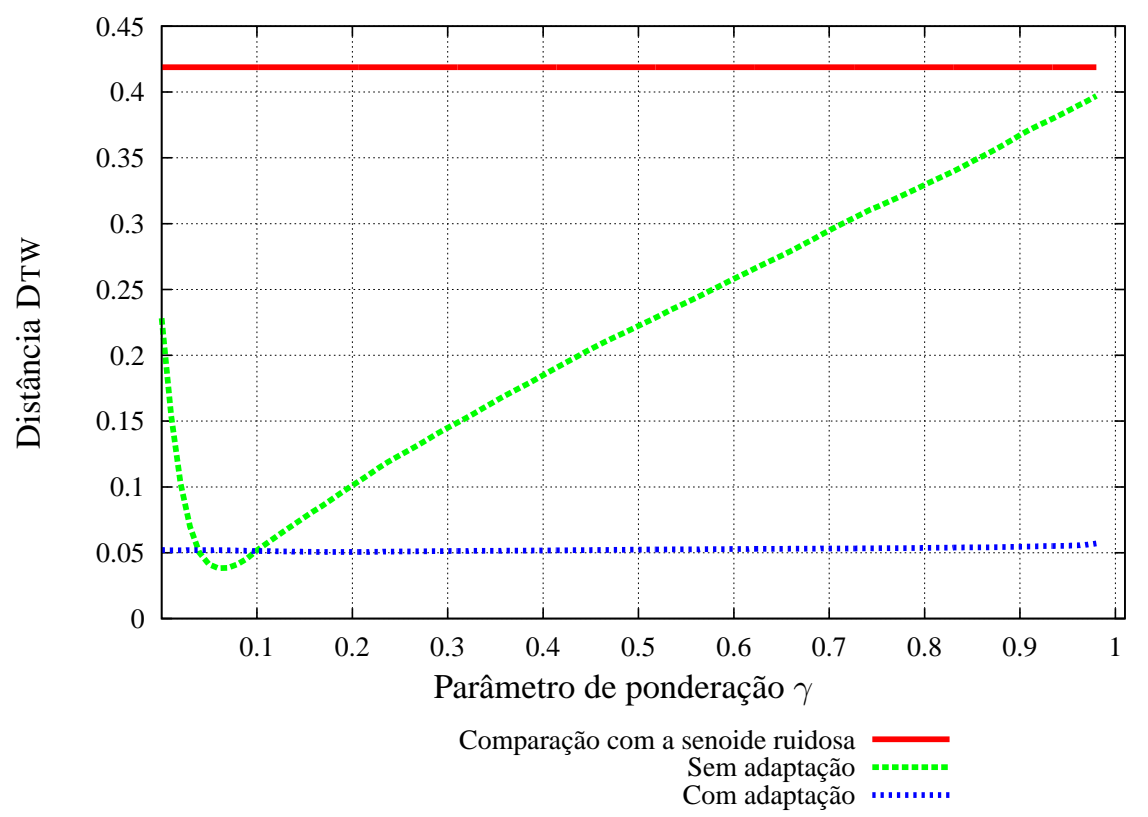

Figura 4.11: Curvas de distância DTw para uma senoide ruidosa de acordo com o parâmetro de ponderação $\gamma$ da arquitetura SONDE, com e sem a técnica de adaptação de viés indutivo atuante

valores inferiores a 0,04 ou superiores a 0,1 , os resultados obtidos a partir da arquitetura SONDE com a técnica de adaptação atuante seriam melhores do que sem a aplicação de tal técnica. Desse experimento pode-se concluir que compensa a utilização de técnicas de adaptação, em virtude de seu melhor desempenho na maior parte do intervalo de parâmetros iniciais e da frequente falta de conhecimento prévio sobre os dados para a minimização da distância do modelo encontrado ao modelo ideal.

Por outro lado, mesmo quando existe a possibilidade de especialistas aplicarem métodos de otimização na etapa de projeto para obtenção de valores parâmetros que produzem resultados inicialmente satisfatórios, tais como o anteriormente mencionado, a variação de comportamento do fluxo de dados pode fazer com que tais parâmetros tornem-se subótimos.

Uma ilustração de um cenário com variação de comportamento é apresentada na Figura 4.12. Primeiramente, obteve-se uma sequência de padrões a partir da execução do comando para o ambiente $\mathrm{R} \tanh (\operatorname{seq}(-20,20$, length $=400))$, com ruído $N(0,0,01)$. Em seguida, empregou-se o método buscaParametro() (Algoritmo 3) a fim de definir um valor inicial para $\gamma$ para prover a menor distância DTw. Em uma segunda etapa, esse valor de $\gamma$ é utilizado no aprendizado de uma sequência composta de $\tanh (\operatorname{seq}(-20,20$, length $=400))$, com ruído $N(0,0,01)$, seguida de outra sequência mais extensa com comportamento diferente do anterior $\cos (\sin (\operatorname{seq}(0,20$, length $=2000)))$, com ruído $N(0,0,25)$. Nessa sequência, aplicou-se a arquitetura SONDE com e sem a técnica de adaptação atuante para comparar suas distâncias DTW. Uma execução dessa segunda etapa é exemplificada na Figura 4.12, na qual apresenta-se, no intervalo de tempo $t \in[1,400]$, um primeiro comportamento caracterizado 
por dois componentes estacionários com um salto entre eles e, nos instantes seguintes, um comportamento senoidal.

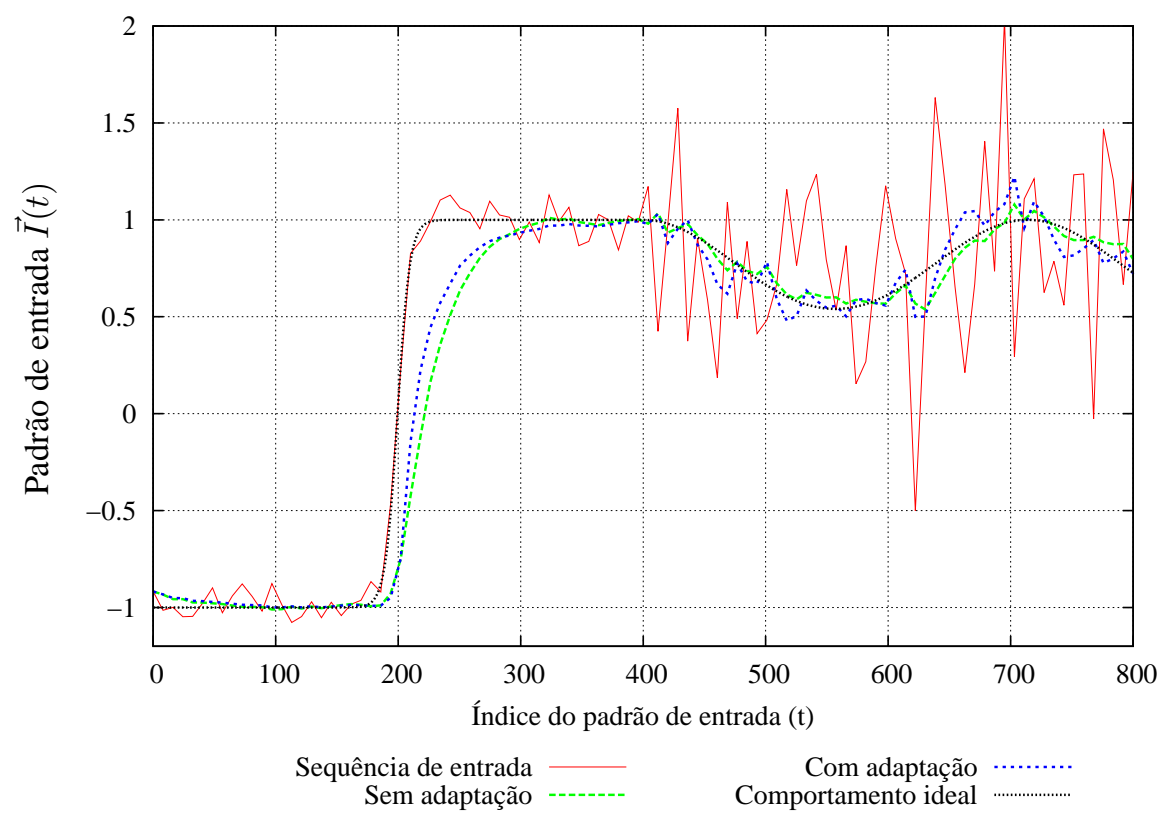

Figura 4.12: Ilustração de sequências de modelo ideal, padrões de entrada ruidosos, protótipos de neurônios da arquitetura SONDE com e sem a técnica de adaptação atuante.

A fim de avaliar os resultados em termos estatisticamente significantes, executou-se esse cenário trinta vezes e aplicou-se um teste de hipótese Wilcoxon-Whitney com a seguinte hipótese nula: a distância DTW entre os protótipos dos neurônios BMU e os dados observados a partir do modelo ideal foi maior ou igual quando a técnica de adaptação de parâmetros estava atuante que quando não estava. Essa hipótese foi rejeitada, com $p-$ value $=0,01597$, comprovando que a abordagem com a técnica de adaptação atuante reduziu a distância DTW em relação à sequência sem ruído, ou seja, ao modelo ideal. Dessa forma, verifica-se a vantagem, em termos de distância $\mathrm{DTW}$, de utilizar uma técnica de adaptação de viés indutivo, mesmo quando bons parâmetros são obtidos no projeto do algoritmo.

A partir desse resultado pode-se generalizar que a manutenção do viés indutivo de parâmetros constante usualmente não será melhor que a sua contínua adaptação caso ocorram variações comportamentais do fenômeno em estudo, em termos de proximidade ao modelo ideal, o que comprova a hipótese desta tese de doutorado. Portanto, caso os valores estabelecidos para parâmetros de uma instância SONDE não sejam adequados, o uso da técnica de adaptação de parâmetros pode evitar a criação de muitos grupos ou grupos pouco representativos.

Por exemplo, considere um fluxo de dados dado por uma sequência senoide ruídosa com baixo ruído $\sigma=0,1$, conforme ilustrado na Figura 4.13. Nesse experimento, a SONDE foi configurada com $\gamma=0,001, \Omega=0,001, \alpha_{0}=0,01$. Apesar desses parâmetros serem válidos e plausíveis para muitas situações, o resultado foi de baixa qualidade: foram obtidos 
dois protótipos e uma distância DTW $=0,232$. Por outro lado, com o uso da técnica de adaptação de parâmetros, obtiveram-se, Figura 4.13 (b), uma distância DTW $=0,11$ e um conjunto de 10 protótipos, que melhor representam os dados.

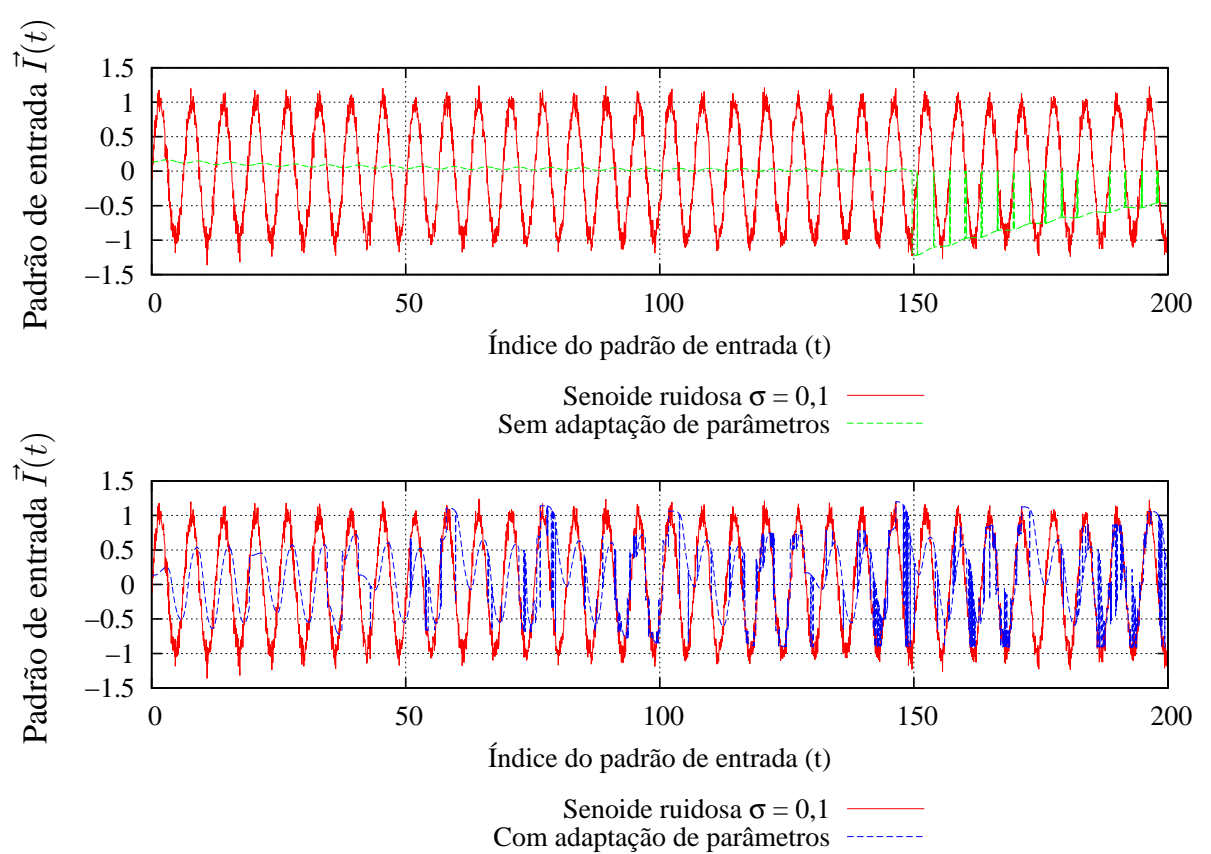

Figura 4.13: Cenário de aprendizado de senoide estacionária

Considere um outro exemplo, uma sequência de dados proveniente de um modelo estocástico $\operatorname{ARIMA}(p=1, d=1, q=0$ ) (parâmetro estatístico $\phi=0.7$ ) (Morettin \& Toloi, 2004), conforme ilustrado na Figura 4.14, Suponha que os parâmetros utilizados no aprendizado são $\gamma=0,01, \Omega=0,01, \alpha_{0}=0,01$. No caso em que a técnica de adaptação do viés indutivo da SONDE não está atuante, obtém-se 1 grupo com distância DTw $=19,952$, por outro lado, com a técnica atuante obtêm-se 8 grupos com distância DTw =0,395.

Por meio desses experimentos, foram observadas as vantagens do emprego de uma técnica de adaptação contínua de viés indutivo de acordo com a variação de comportamento do fenômeno em estudo. Conforme o exemplo apresentado na Figura 4.11, existe uma grande chance de especialistas não serem capazes de atribuir valores para parâmetros de agrupamento próximos aos teoricamente ótimos. Isso ocorre tanto no caso do emprego de uma heurística de otimização para encontrar tais parâmetros, quanto no uso de valores para parâmetros aparentemente plausíveis em casos que não há amostras disponíveis na etapa de projeto (Figura 4.13). Adicionalmente, mesmo quando parâmetros ótimos são encontrados, uma eventual variação de comportamento pode torná-los subótimos (Figura 4.12). Em todos os casos, a adaptação do viés indutivo é necessária para garantir a contínua obtenção de conhecimento a partir da análise de fluxos de dados. 

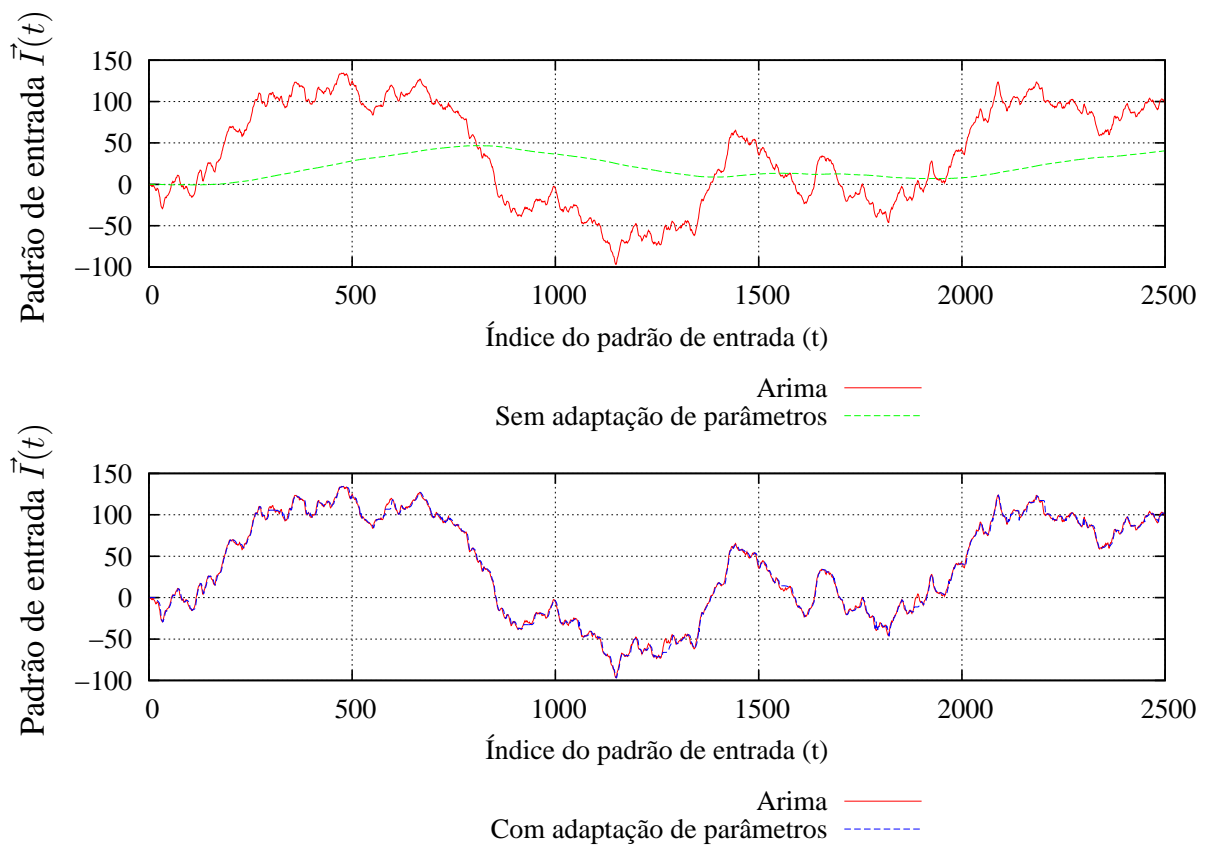

Figura 4.14: Cenário de aprendizado de sequência produzida a partir de um modelo estocástico $\operatorname{Arima}(1,1,0)$

\subsubsection{Discussão dos resultados obtidos a partir da terceira abordagem}

A necessidade de prover adaptação e resposta imediatas à coleta contínua de dados para fenômenos com variação de comportamento motivou o projeto da terceira abordagem de adaptação de viés indutivo para algoritmos de agrupamento de fluxos de dados. Essa abordagem foi inspirada em propriedades que relacionam a avaliação de estabilidade dos dados coletados com a adaptação do fator de ponderação de médias móveis exponenciais ponderadas (MMEP), que constituem o viés indutivo da arquitetura SONDE.

A partir dos experimentos realizados pôde-se comprovar a hipótese desta tese ao observar claramente a necessidade e as vantagens da adaptação contínua do viés indutivo. Essa abordagem buscou avaliar o comportamento de fenômenos a partir da avaliação direta dos dados, sem a introdução de suposições (e.g., que fenômenos podem ser modelados a partir de cadeias de Markov), aproximações (e.g., uso de propriedades de convergência) e outras simplificações que induzem a erros, conforme realizado na primeira abordagem. A mesma comparação é valida em relação à segunda abordagem, com a desvantagem adicional de que o uso de blocos de dados interferem na compreensão contínua do fenômeno observado.

Em relação às propriedades teóricas definidas no Capítulo 2, pode-se observar que, devido às características da arquitetura SONDE, a primeira abordagem respeita as propriedades de Completude em Fluxos de Dados (Propriedade 5 e Coerência (Propriedade 7, porém não respeita as propriedades de Invariância Espaço-temporal (Propriedade 4) e Consistência (Propriedade 6).

Enfim, embora sejam necessários experimentos adicionais para a verificação e compa- 
ração de resultados com outros algoritmos de agrupamento, esta terceira abordagem apresenta uma nova técnica para adaptar o viés indutivo de acordo com a variação de comportamento em dados, conforme a abordagem idealizada no objetivo desta tese.

\subsection{Considerações finais}

Neste capítulo apresentaram-se as atividades realizadas para atingir o objetivo desta tese: o projeto de uma abordagem de adaptação do viés indutivo de algoritmos de agrupamento para fenômenos que apresentam variação comportamental. Tal abordagem foi idealizada para ser composta de: i) um algoritmo de agrupamento de fluxos de dados; ii) uma medida para avaliar variações comportamentais presentes em fluxos de dados; iii) e uma abordagem de adaptação de viés indutivo por meio de parâmetros de acordo com variações de comportamento presentes em fluxos de dados.

Dentre as atividades realizadas para atingir esse objetivo destacam-se: o levantamento de trabalhos da área de detecção de novidades e proposta de uma hierarquia de organização de técnicas considerando a necessidade de avaliar o comportamento de fenômenos (Capítulo 3); o estudo de algoritmos de agrupamento de fluxos de dados (Capítulo 2); a proposta de uma formalização para o problema de agrupamento de fluxos de dados; a proposta de uma arquitetura de rede neural artificial denominada Self-Organizing Novelty Detector (Albertini \& Mello, 2007); e a proposta de um método de estimação de cadeias de Markov integrado à arquitetura SONDE para avaliar continuamente a variação de comportamento por meio da entropia de Shannon de cadeias de Markov (Mello et al., 2010).

Essas atividades apoiaram o projeto e desenvolvimento de três abordagens da forma idealizada no objetivo da tese. Inicialmente, buscou-se adaptar continuamente o viés indutivo da arquitetura SONDE por meio de um método de otimização estocástica; em seguida, devido a dificuldades em relacionar a variação de comportamento e os parâmetros dessa arquitetura optou-se por utilizar técnicas tradicionais para agrupamento, i.e., K-médias, e adaptar o viés indutivo por meio de um método de busca exaustiva, ao custo de não realizar sua adaptação contínua; continuou-se, portanto, estudos para desenvolver uma técnica de adaptação contínua para a SONDE, os quais resultaram na proposta de uma terceira abordagem, baseada na relação entre a estabilidade de dados e o fator de ponderação das equações de aprendizado da SONDE. Essa terceira e última abordagem, por fim, permitiu a comprovação da hipótese desta tese.

A execução dessas atividades e o desenvolvimento dessas propostas resultaram nas seguintes publicações:

1 - Albertini, M. K. e Mello, R. F., A Self-Organizing Neural Network for Detecting Novelties. In: ACM Symposium on Applied Computing, 2007, Seul, Coreia. ACM Symposium on Applied Computing, 2007. 
DOI: $10.1145 / 1244002.1244110$

2 - Albertini, M. K. e Mello R. F., Intelligent Systems for Automated Learning and Adaptation: Emerging Trends and Applications, capítulo intitulado A Self-Organizing Neural Network to Approach Novelty Detection, p. 49-71. IGI Global, 2008.

DOI: 10.4018/978-1-60566-798-0.ch003

3 - Albertini, M. K.; Li, K. C. e Mello, R. F., A Novel Approach to Quantify Novelty Levels Applied on Ubiquitous Music Distribution, p. 150-155, IEEE Asia-Pacific Services Computing Conference, Yilan, Taiwan, 2008.

DOI: 10.1109/APSCC.2008.238

4 - Mello, R. F.; dos Santos, M. L.; e Albertini, M. K., Variation Detection applied in User Signature Verification. Revista de Informática Teórica e Aplicada. vol. 17, n. 1 (2010). URL: http://seer.ufrgs.br/rita/article/view/rita_v17_n1_p71

5 - Gouveia, L. T.; Costa, L. F.; Senger, L. J.; Albertini, M. K.; e Mello, R. F., An Entropy-Based Approach to Analyze and Classify Mineral Aggregates. Journal of Computing in Civil Engineering, v. 1, p. 1-48, 2010. JCR 2010: 0,9

DOI: 10.1061/(ASCE)CP.1943-5487.0000071

Recentemente, o seguinte artigo foi avaliado e aceito para publicação:

6 - Albertini, M. K. e Mello, R. F, Data Stream Dynamic Clustering Supported by Markov Chain Isomorphisms, Intelligent Data Analysis Journal. v. 17 (3), 2013. JCR 2010: 0,412

Adicionalmente, os seguintes manuscritos foram enviados para avaliação em revistas internacionais:

7 - Albertini, M. K.; Stojmenovic, I. ; Mello, R. F, Feature Extraction Based on ExponentialWeighted Higher-Order Local Auto-Correlation: An Approach to Improve Data Characterization

8 - Albertini, M. K. e Mello, R. F, Energy-based Function to Evaluate Data Stream Clustering

Por fim, o seguinte manuscrito encontra-se em processo de preparação:

9 - Albertini, M. K. e Mello, R. F, Analysis of Data Stream Clustering as a Problem of Partitioning Infinite Sequences. 


혼

\section{Conclusões e trabalhos futuros}

O método não supervisionado mais utilizado para a análise de fenômenos pouco conhecidos é denominado Agrupamento de Dados (Xu \& Wunsch, 2005). Seu objetivo é encontrar e distinguir propriedades relevantes a partir da avaliação da similaridade, ou dissimilaridade, de dados (Jain et al., 1999; Jain, 2010).

Atualmente, o processo de agrupamento tem exibido sérias limitações em sua aplicação a fluxos de dados, devido às exigências impostas pelas variações comportamentais e a coleta ininterrupta de dados (Pavlidis et al., 2011). Essas variações comportamentais requerem a adaptação contínua do viés indutivo estabelecido no projeto do algoritmo de agrupamento.

Embora exista uma extensa literatura em agrupamento de fluxos de dados e caracterização de variações comportamentais, a atual lacuna apresentada pela escassez de estudos sobre a adaptação do viés indutivo de algoritmos e a necessidade de compreender fenômenos observados a partir de fluxos de dados com variação de comportamento motivaram a definição da hipótese desta tese de doutorado:

Hipótese: Adaptando o viés indutivo de algoritmos de agrupamento de fluxos de dados de acordo com variações comportamentais do fenômeno em estudo pode-se garantir a contínua obtenção de conhecimento. 
A partir dos estudos e experimentos realizados e da hipótese estabelecida, definiu-se como objetivo desta tese:

Objetivo: Projetar e desenvolver uma abordagem de adaptação do viés indutivo de parâmetros de algoritmos de agrupamento de fluxos de dados, considerando fenômenos que apresentam variação comportamental.

Para comprovar a hipótese e cumprir o objetivo estabelecido, realizou-se diversas atividades de pesquisa, dentre as quais se destacam os estudos de três propostas para o projeto da abordagem idealizada.

Os estudos realizados para a primeira abordagem basearam-se, conforme o diagrama apresentado na Figura 1.2, em: i) projeto de uma arquitetura de rede neural artificial autoorganizável denominada Self-Organizing Novelty Detector (SONDE) para agrupar dados de maneira adaptativa e incremental (Albertini \& Mello, 2007); ii) medição de variações comportamentais por meio da estimação de cadeias de Markov (Seneta, 2006) e da entropia de Shannon (1948); e iii) projeto de uma técnica de adaptação de viés indutivo por meio da minimização da variação de entropia de cadeias de Markov ocorrida devido aos parâmetros que constituem o viés da arquitetura SONDE.

Nessa primeira abordagem, descrita na Seção 4.6, a arquitetura projetada SONDE permitiu representar grupos por meio de equações de aprendizado baseadas em proximidade espacial entre elementos, as quais possuem parâmetros que constituem seu viés indutivo. A representação de grupos por meio de neurônios da SONDE permitiu a estimação da matriz de probabilidades de transições de cadeias de Markov, que é a base para o cálculo de uma medida de caracterizacão comportamental de fenômenos baseada em variacão da entropia (Albertini \& Mello, 2008; Mello et al., 2010; de Gouveia et al., 2011). Essa medida foi utilizada no projeto da técnica de adaptação de viés indutivo inspirada em um método de otimização estocástico de gradiente descendente. Nessa técnica, a função gradiente da variação de entropia em relação aos parâmetros da SONDE indicaria a intensidade em que tais parâmetros seriam adaptados. Porém, apesar da concentração de esforços para o desenvolvimento e refinamento dessa abordagem, ela não foi bem sucedida. Isso ocorreu principalmente devido a limitações encontradas na obtenção da relação entre variações de entropia e os parâmetros da SONDE, necessária à definição da função de gradiente utilizada no método de otimização.

Posteriormente, buscou-se outras alternativas para definir a relação entre o algoritmo de agrupamento e a adaptação do viés indutivo. Essa busca resultou em uma segunda abordagem que constituiu, conforme o diagrama na Figura 1.2, da: i) utilização do algoritmo de agrupamento para conjuntos tradicionais de dados K-médias (Hartigan, 1975) para a produção 
de partições em blocos de subsequências; ii) estimação de propriedades assintóticas de cadeias de Markov para a verificação de equivalência de comportamento entre partições consecutivas (Ornstein, 1989; Seneta, 2006); e iii) aplicação de um algoritmo de otimização que define o número de grupos para o algoritmo K-médias de acordo com a medida Silhueta (Rousseeuw, 1987).

Essa segunda abordagem, denominada Data Stream Dynamic Clustering, foi bem sucedida em adaptar o viés indutivo de acordo o reconhecimento da variação de comportamento entre blocos de dados (Albertini \& Mello, 2013). Contudo, conforme mencionado na hipótese desta tese, para manter a efetiva obtenção de conhecimento sobre fenômenos é necessária a contínua avaliação dos dados. Dessa forma, o uso de algoritmos de agrupamento baseados em blocos de dados, que não distinguem a ordem de coleta de dados pertencentes a um mesmo bloco, prejudica a compreensão de variações de comportamento de fenômenos.

A adaptação contínua do viés indutivo de algoritmos para efetivamente manter atualizado o conhecimento sobre o comportamento do fenômeno em estudo motivou estudos para a proposta de uma terceira abordagem definida no objetivo desta tese. Esses estudos basearam-se, conforme a Figura 1.2, em: i) utilização da arquitetura SONDE como algoritmo de agrupamento; ii) emprego de uma medida de estabilidade de comportamento presente no fluxo de dados; e iii) projeto de uma técnica de adaptação de viés indutivo baseada na relação entre a medida de estabilidade e os parâmetros das equações de aprendizado de estruturas de conhecimento da arquitetura SONDE,

A proposta da terceira abordagem partiu da associação dos parâmetros das equações de aprendizado da SONDE à necessidade de representar a dependência temporal de acordo com a estabilidade de comportamento observada nos dados. Para isso, projetou-se uma técnica de adaptação baseada em uma função de mapeamento entre a medida de estabilidade de dados e os parâmetros das equações da arquitetura SONDE. Essa técnica atua sobre os parâmetros de forma a aumentar a memória das equações de aprendizado, caso dados apresentem maior estabilidade e diminuir, caso contrário.

Resultados obtidos a partir de experimentos realizados com fluxos de dados sintéticos, utilizando a terceira abordagem proposta, permitiram verificar as vantagens da adaptação contínua do viés indutivo de agrupamento. A partir desses resultados (Figuras 4.11, 4.12 e 4.13) pôde-se comprovar a hipótese desta tese ao observar claramente a necessidade e as vantagens da adaptação contínua do viés indutivo. Primeiramente, observa-se, Figura 4.11 que apenas em pequenos intervalos do espaço de parâmetros o uso da técnica de adaptação de viés indutivo é inferior ao estático. Em segundo lugar, Figura 4.12, pôde-se verificar que, mesmo quando o algoritmo de agrupamento é iniciado com parâmetros teoricamente ótimos, variações de comportamento podem torná-los subótimos e que, em tal situação, o uso da técnica de adaptação de parâmetros é vantajosa. Em terceiro lugar, comumente, em agrupamento não há modelo ideal para estabelecer um valor de referência ótimo para parâmetros de aprendizado, 
portanto, mesmo a partir do uso de parâmetros aparentemente adequados, segundo conhecimento prévio de especialista, o resultado obtido pode ser muito inferior ao obtido a partir do emprego de uma técnica de adaptação (Figura 4.13).

Além do projeto dessas três abordagens, resume-se a seguir as contribuições adicionais desta tese de doutorado em:

1. proposta de uma hierarquia de organização de técnicas de detecção de novidades considerando a necessidade de avaliar o comportamento de fenômenos (Capítulo 3);

2. a proposta de uma formalização para o problema de agrupamento de fluxos de dados;

3. a proposta de propriedades básicas para a análise e comparação de algoritmos de agrupamento de fluxos de dados (Capítulo 2);

4. a proposta de uma arquitetura de rede neural artificial denominada Self-Organizing Novelty Detector (Albertini \& Mello, 2007);

5. e a proposta de um método de estimação de cadeias de Markov integrado à arquitetura SONDE para avaliar continuamente a variação de comportamento por meio da entropia de Shannon (Mello et al., 2010).

A comprovação da hipótese apresentada nesta tese de doutorado motiva a realização de trabalhos futuros para ampliar a formalização de propriedades de algoritmos e estender a adaptação do viés indutivo de algoritmos de agrupamento de fluxos de dados. A execução desses trabalhos futuros está prevista para um estágio de pós-doutorado a ser realizado no Instituto de Ciências Matemáticas e de Computação da Universidade de São Paulo, com o apoio da Fundação de Amparo à Pesquisa do Estado de São Paulo, processo n. 2011/19459-8, sob supervisão do prof. Rodrigo Fernandes de Mello.

É possível especular que, no futuro, tais estudos permitam o acúmulo de conhecimento genérico o suficiente sobre o problema de agrupamento para auxiliar na composição de uma Teoria de Agrupamento e complementar o conhecimento atual sobre esse problema (Meila, 2005; Antzoulatos \& Vrahatis, 2011). 


\section{Referências Bibliográficas}

Aggarwal, C. C.; Han, J.; Wang, J.; Yu, P. S. (2003). A framework for clustering evolving data streams. Proceedings of the 29th international conference on Very Large Data Bases, v. 29, p. 81-92, Berlim, Alemanha. VLDB Endowment.

Al-Subaie, M.; Zulkernine, M. (2006). Efficacy of hidden Markov models over neural networks in anomaly intrusion detection. Proceedings of the 30th Annual International Computer Software and Applications Conference, p. 325-332, Washington, EUA. IEEE Computer Society.

Albertini, M. K.; Mello, R. F. (2005). Estudo e proposta de um suporte transparente de alta disponibilidade distribuído. Proceedings of 4th IEEE International Information and Telecommunication Technologies Symposium, p. 1-7, Florianópolis, SC.

Albertini, M. K.; Mello, R. F. (2007). A self-organizing neural network for detecting novelties. Proceedings of the 2007 ACM symposium on Applied computing, p. 462-466, Nova Iorque, EUA. ACM.

Albertini, M. K.; Mello, R. F. (2008). Intelligent Systems for Automated Learning and Adaptation: Emerging Trends and Applications, chapter A Self-Organizing Neural Network to Approach Novelty Detection, p. 19. IGI Global, Sarawak, Malásia.

Albertini, M. K.; Mello, R. F. (2013). Data Stream Dynamic Clustering Supported by Markov Chain Isomorphisms. Intelligent Data Analysis, v.17, p.1-30.

Alcock, R.; Manolopoulos, Y.; others (1999). Time-series similarity queries employing a feature-based approach. 7th Hellenic conference on informatics, Ioannina, Grécia, p. 1-9.

Alizadeh, A. A.; Eisen, M. B.; Davis, E. E.; Ma, C.; Lossos, I. S.; Rosenwald, A.; Boldrick, J. C.; Sabet, H.; Tran, T.; Yu, X.; Powell, J. I.; Yang, L.; Marti, G. E.; Moore, T.; 
Hudson, J.; Lu, L.; Lewis, D. B.; Tibshirani, R.; Sherlock, G.; Chan, W. C.; Greiner, T. C.; Weisenburger, D. D.; Armitage, J. O.; Warnke, R.; Levy, R.; Wilson, W.; Grever, M. R.; Byrd, J. C.; Botstein, D.; Brown, P. O.; Staudt, L. M. (2000). Distinct types of diffuse large B-cell lymphoma identified by gene expression profiling. Nature, v.403, n.6769, p.503-511.

Allcock, B.; Foster, I.; Nefedova, V.; Chervenak, A.; Deelman, E.; Kesselman, C.; Lee, J.; Sim, A.; Shoshani, A.; Drach, B.; others (2001). High-performance remote access to climate simulation data: A challenge problem for data grid technologies. Parallel Computing, v. 29, p. $1335-1356$.

Andrews, G. E.; Askey, R.; Roy, R. (2001). Special Functions. Cambridge University Press, Cambridge, Reino Unido.

Antzoulatos, G.; Vrahatis, M. (2011). a-clusterable sets. Gunopulos, D.; Hofmann, T.; Malerba, D.; Vazirgiannis, M., editores, Machine Learning and Knowledge Discovery in Databases, v. 6911 de Lecture Notes in Computer Science, p. 108-123. Springer.

Banerjee, A.; Merugu, S.; Dhillon, I.; Ghosh, J. (2005). Clustering with Bregman divergences. Journal of Machine Learning Research, v.6, p.1705-1749.

Bardo, M. T.; Donohew, R. L.; Harrington, N. G. (1996). Psychobiology of novelty seeking and drug seeking behavior. Behavioural Brain Research, v.77, n.1-2, p.23-43.

Barnett, V.; Lewis, T. (1994). Outliers in statistical data. John Wiley \& Sons, Chichester, EUA, $3^{\mathrm{a}}$ edição.

Ben-David, S.; Ackerman, M. (2009). Measures of clustering quality: A working set of axioms for clustering. Advances in Neural Information Processing Systems 21, p. 121-128.

Ben-David, S.; Von Luxburg, U.; Pál, D. (2006). A sober look at clustering stability. Learning Theory, v.4005, p.5-19.

Bensalah, Y. (2000). Steps in applying extreme value theory to finance: A review. Notas de trabalho 00-20, Bank of Canada. Disponível em: http://ideas.repec.org/p/bca/bocawp/00-20.html. Acesso em: 23 de fevereiro de 2012.

Bertarelli, S.; Censolo, R. (2000). Preference for novelty and price behaviour. Working Papers 383, Dipartimento Scienze Economiche, Università di Bologna, Bolonha, Itália.

Biehl, M.; Ghosh, A.; Hammer, B. (2006). Learning vector quantization: The dynamics of Winner-Takes-all algorithms. Neurocomputing, v.69, n.7-9, p.660 - 670.

Bishop, C. M. (1995). Neural Networks for Pattern Recognition. Oxford University Press, Nova Iorque, EUA, $12^{\text {a }}$ edição. 
Boriah, S.; Kumar, V.; Steinbach, M.; Potter, C.; Klooster, S. (2008). Land cover change detection: a case study. Proceedings of the 14th ACM International Conference on Knowledge Discovery and Data Mining, p. 857-865, Nova Iorque, EUA. ACM.

Box, G. E. P.; Jenkins, G. M. (1976). Time Series Analysis, Forecasting, and Control. Holden-Day, São Francisco, EUA.

Boynton, G. R. (2010). Twitter data stream: American killed Israeli. Publicado em http://ir.uiowa.edu/polisci_nmp/71/. Acessado em 23 de fevereiro de 2012.

Brazdil, P. (2009). Metalearning - Applications to Data Mining. Cognitive Technologies. Springer.

Brazdil, P.; Giraud-Carrier, C.; Soares, C.; Vilalta, R.; João, G.; Castillo, G. (2009). Bias management in time-changing data streams. Metalearning, Cognitive Technologies, p. 91-107. Springer.

Bush, V. (1931). The differential analyzer. a new machine for solving differential equations. Journal of the Franklin Institute, v.212, n.4, p.447 - 488.

Carlsson, G.; Mémoli, F. (2010a). Characterization, Stability and Convergence of Hierarchical Clustering Methods. Journal of Machine Learning Research, v.11, p.1425-1470.

Carlsson, G.; Mémoli, F. (2010b). Multiparameter hierarchical clustering methods. Classification as a Tool for Research, Studies in Classification, Data Analysis, and Knowledge Organization, p. 63-70. Springer.

Carlsson, G.; Mémoli, F. (2008). Persistent clustering and a theorem of J. Kleinberg. ArXiv e-prints, v.1, p.17. http://adsabs.harvard.edu/abs/2008arXiv0808.2241C.

Carpenter, G. A.; Grossberg, S.; Rosen, D. B. (1991). ART 2-A: an adaptive resonance algorithm for rapid category learning and recognition. Neural Networks, v.4, n.4, p.493-504.

Ciuperca, G.; Girardin, V. (2007). Estimation of the entropy rate of a countable Markov chain. Communications in Statistics - Theory and Methods, v.36, n.14, p.2543-2557.

Conover, M.; Ratkiewicz, J.; Francisco, M.; Gonçalves, B.; Flammini, A.; Menczer, F. (2011). Political polarization on Twitter. Proceedings of the 5th International Conference on Weblogs and Social Media, Barcelona, Espanha.

Cortes, C.; Pregibon, D. (2001). Signature-based methods for data streams. Data Mining and Knowledge Discovery, v.5, p.167-182.

Daszykowski, M.; Walczak, B.; Massart, D. L. (2002). On the Optimal Partitioning of Data with K-Means, Growing K-Means, Neural Gas, and Growing Neural Gas. Journal of Chemical Information and Computer Sciences, v.42, n.6, p.1378-1389. 
Daura, X.; Jaun, B.; Seebach, D.; van Gunsteren, W. F.; Mark, A. E. (1998). Reversible peptide folding in solution by molecular dynamics simulation. Journal of Molecular Biology, v.280, n.5, p.925-932.

de Gouveia, L. T.; da Fontoura Costa, L.; Senger, L. J.; Albertini, M. K.; de Mello, R. F. (2011). Entropy-based approach to analyze and classify mineral aggregates. Journal of Computing in Civil Engineering, v.25, n.1, p.75-84.

Dehaspe, L.; Raedt, L. D. (1996). DLAB: A Declarative Language Bias Formalism. In Proceedings of the International Symposium on Methodologies for Intelligent Systems, $\mathrm{p}$. 613-622, Charlotte, EUA. Springer.

Dewdney, P.; Hall, P.; Schilizzi, R.; Lazio, T. (2009). The square kilometre array. Proceedings of the IEEE, v.97, n.8, p.1482-1496.

Ester, M.; Kriegel, H.; Sander, J.; Xu, X. (1996). A density-based algorithm for discovering clusters in large spatial databases with noise. Proceedings of Knowledge Discovery and Data Mining, v. 96, p. 226-231, Portland, EUA.

Fisher, R. A.; Tippett, L. (1928). Limiting forms for the frequency distribution of the largest or smallest member of a sample. Proceedings of Cambridge Philosophical Society, v. 24, p. 180-190, Cambridge, Reino Unido.

Flexer, A.; Pampalk, E.; Widmer, G. (2005). Novelty detection based on spectral similarity of songs. Proceedings of 6th International Conference on Music Information Retrieval, p. 260-263, Londres, Reino Unido.

Frank, A.; Asuncion, A. (2010). UCI machine learning repository. Disponível em: http://archive.ics.uci.edu/ml/. Acesso em: 1o de março de 2012.

Fritzke, B. (1995). A growing neural gas network learns topologies. Advances in Neural Information Processing Systems 7, p. 625-632. MIT Press, Cambridge, Reino Unido.

Gamon, M. (2006). Graph-based text representation for novelty detection. Proceedings of TextGraphs: the Second Workshop on Graph Based Methods for Natural Language Processing, p. 17-24, Nova Iorque, EUA. Association for Computational Linguistics.

Giorgino, T. (2009). Computing and Visualizing Dynamic Time Warping Alignments: The dtw Package. Journal of Statistical Software, v.31, n.7, p.1-24.

Golab, L.; Özsu, M. T. (2003). Processing sliding window multi-joins in continuous queries over data streams. Proceedings of the 29th international conference on Very large data bases, v. 29, p. 500-511, Berlim, Alemanha. VLDB Endowment. 
Golub, T. R.; Slonim, D. K.; Tamayo, P.; Huard, C.; Gaasenbeek, M.; Mesirov, J. P.; Coller, H.; Loh, M. L.; Downing, J. R.; Caligiuri, M. A.; Bloomfield, C. D.; Lander, E. S. (1999). Molecular classification of cancer: class discovery and class prediction by gene expression monitoring. Science, v.286, n.5439, p.531-537.

Guha, S.; Meyerson, A.; Mishra, N.; Motwani, R.; O'Callaghan, L. (2003). Clustering data streams: Theory and practice. v. 15, p. 515-528, Piscataway, EUA. IEEE Educational Activities Department.

Hartigan, J. (1975). Clustering Algorithms. John Wiley and Sons, Nova Iorque, EUA.

Hartigan, J.; Wong, M. (1979). Algorithm as 136: A k-means clustering algorithm. Journal of the Royal Statistical Society. Series C (Applied Statistics), v.28, n.1, p.100-108.

Hasselmann, K. (1997). Multi-pattern fingerprint method for detection and attribution of climate change. Climate Dynamics, v.13, n.9, p.601-611.

Hawking, S. (2003). On the Shoulders of Giants: The Great Works of Physics and Astronomy. Running Press Book Publisher, Reino Unido.

Heas, P.; Datcu, M. (2005). Modeling trajectory of dynamic clusters in image time-series for spatio-temporal reasoning. Geoscience and Remote Sensing, IEEE Transactions on, v.43, n.7, p.1635- 1647 .

Hey, T.; Tansley, S.; Tolle, K., editores (2009). The Fourth Paradigm: Data-Intensive Scientific Discovery. Microsoft Research, Redmond, EUA.

Hinneburg, A.; Keim, D. A. (1999). Optimal grid-clustering: Towards breaking the curse of dimensionality in high-dimensional clustering. Proceedings of the 25th International Conference on Very Large Data Bases, p. 506-517, São Francisco, EUA. Morgan Kaufmann Publishers Inc.

Hodge, V.; Austin, J. (2004). A survey of outlier detection methodologies. Artificial Intelligence Review, v.22, n.2, p.85-126.

Holt, C. C. (2004). Forecasting seasonals and trends by exponentially weighted moving averages. International Journal of Forecasting, v.20, n.1, p.5-10.

Hughes, G.; Murray, J.; Kreutz-Delgado, K.; Elkan, C. (2002). Improved disk-drive failure warnings. IEEE Transactions on Reliability, v.51, n.3, p.350-357.

Hulten, G.; Spencer, L.; Domingos, P. (2001). Mining time-changing data streams. Proceedings of the seventh ACM International Conference on Knowledge Discovery and Data Mining, p. 97-106, São Francisco, EUA. ACM. 
Ilango, M.; V., M. (2010). A survey of grid based clustering algorithms. International Journal of Engineering Science and Technology, v.2, p.8.

Ishii, R. P.; Rios, R. A.; Mello, R. F. (2011). Classification of time series generation processes using experimental tools: a survey and proposal of an automatic and systematic approach. International Journal of Computational Science and Engineering, v.6, n.4, p.217-237.

Itti, L.; Baldi, P. (2005). A principled approach to detecting surprising events in video. Proceedings of the 2005 IEEE Computer Society Conference on Computer Vision and Pattern Recognition, v. 1, p. 631-637, Washington, DC, EUA. IEEE Computer Society.

Jain, A. (2010). Data clustering: 50 years beyond K-means. Pattern Recognition Letters, v.31, n.8, p.651-666.

Jain, A.; Murty, M.; Flynn, P. (1999). Data clustering: a review. ACM Computing Surveys, v.31, n.3, p.264-323.

Jain, A. K.; Dubes, R. C. (1988). Algorithms for clustering data. Prentice Hall, Nova Jersey, EUA.

Kaluza, B.; Mirchevska, V.; Dovgan, E.; Lustrek, M.; Gams, M. (2010). An agent-based approach to care in independent living. Ambient Intelligence, v. 6439 de Lecture Notes in Computer Science, p. 177-186. Springer.

Kant, I. (1781). Crítica da razão pura. Anselmi, L. A. C. and Lubisco, F., São Paulo, Brasil, 2007 edição. Tradução Editora Ícone.

Kaufmann, G. (2004). Two kinds of creativity - but which ones? Creativity and Innovation Management, v.13, n.3, p.154-165.

Kifer, D.; Ben-David, S.; Gehrke, J. (2004). Detecting change in data streams. Proceedings of the Thirtieth international conference on Very Large Data Bases, v. 30, p. 180-191, Toronto, Canadá. VLDB Endowment.

Kleinberg, J. (2002). An impossibility theorem for clustering. Proceedings of Advances in Neural Information Processing Systems 15, p. 463, Londres, Reino Unido. The MIT Press.

Kohonen, T. (1997). Self-organizing maps. Springer, Secaucus, EUA.

Korting, T. S.; Fonseca, L. M. G.; Escada, M. I. S.; Camara, G. (2009). GeoDMA Um sistema para mineração de dados de sensoriamento remoto. Simpósio Brasileiro de Sensoriamento Remoto, Natal, Brasil.

Kramer, W. T. C.; Shoshani, A.; Agarwal, D. A.; Draney, B. R.; Jin, G.; Butler, G. F.; Hules, J. A. (2004). Deep scientific computing requires deep data. IBM Journal of Research and Development, v.48, n.2, p.209-232. 
Kullback, S. (1959). Information theory and statistics. John Wiley and Sons., Nova Iorque, EUA.

Lau, K.-M.; Weng, H. (1995). Climate signal detection using wavelet transform: How to make a time series sing. Bulletin of the American Meteorological Society, v.76, n.12, p.2391-2402.

Lauer, M. (2001). A mixture approach to novelty detection using training data with outliers. Proceedings of the 12th European Conference on Machine Learning, p. 300-311, Londres, Reino Unido. Springer.

Lindstrom, P.; Delany, S.; Mac Namee, B. (2010). Handling Concept Drift in a Text Data Stream Constrained by High Labelling Cost. Proceedings of the Twenty-Third International Florida Artificial Intelligence Research Society Conference, p. 52, Daytona Beach, EUA.

Liénard, A. (1928). Étude des oscillations entretenues. Revue générale de l'électricité, v.23, p.901-912,946-954.

Lukashin, A.; Lukashev, M.; Fuchs, R. (2003). Topology of gene expression networks as revealed by data mining and modeling. Bioinformatics, v.19, n.15, p.1909.

Luterbacher, J.; Dietrich, D.; Xoplaki, E.; Grosjean, M.; Wanner, H. (2004). European seasonal and annual temperature variability, trends, and extremes since 1500. Science, v.303, n.5663, p.1499-1503.

Ma, J.; Perkins, S. (2003). Online novelty detection on temporal sequences. Proceedings of the ninth ACM SIGKDD International Conference on Knowledge Discovery and Data Mining, p. 613-618, Nova Iorque, EUA. ACM Press.

Mann, H. B.; Whitney, D. R. (1947). On a test of whether one of two random variables is stochastically larger than the other. The Annals of Mathematical Statistics, v.18, n.1, p.50-60.

Markou, M.; Singh, S. (2003). Novelty detection: a review - part 1: statistical approaches, part2: neural network based approaches. Signal Processing, v.83, n.12, p.2481-2497, 2499-2521.

Marsland, S. (2002). On-line Novelty Detection Through Self-Organisation, With Application to Inspection Robotics. Tese (Doutorado), University of Manchester, Inglaterra.

Marsland, S.; Shapiro, J.; Nehmzow, U. (2002). A self-organising network that grows when required. Neural Networks, v.15, n.8-9, p.1041-1058.

Martinetz, T. M.; Schulten, K. J. (1994). Topology representing networks. Neural Networks, v.7, n.3, p.505-522. 
Masud, M.; Chen, Q.; Gao, J.; Khan, L.; Han, J.; Thuraisingham, B. (2010). Classification and Novel Class Detection of Data Streams in a Dynamic Feature Space. Machine Learning and Knowledge Discovery in Databases, v.6322, p.337-352.

Meila, M. (2005). Comparing clusterings: an axiomatic view. Proceedings of the 22nd International Conference on Machine Learning, p. 577-584, Bonn, Alemanha. ACM.

Mello, R. F. d.; Santos, M. L. d.; Albertini, M. K. (2010). Variation detection applied in user signature verification. Revista de Informática Teórica e Aplicada, v.17, p.71-92.

Middleton, E. (1996). Adaptation level and 'animal spirits'. Journal of Economic Psychology, v.17, n.4, p.479-498.

Mitchell, T. M. (1997). Machine Learning. McGraw-Hill, Nova Iorque, EUA.

Morettin, P. A.; Toloi, C. M. (2004). Análise de Séries Temporais. Editora Edgard Blücher, São Paulo, Brasil.

Mota, J. S.; Câmara, G.; Escada, M. I. S.; Bittencourt, O.; Fonseca, L. M. G.; Vinas, L. (2009). Case-based reasoning for eliciting the evolution of geospatial objects. Proceedings of the 9th International Conference on Spatial Information Theory, p. 405-420, Aber Wrac'h, França. Springer.

Moura, A. D. (1996). Von Neumann e a previsão numérica de tempo e clima. Estudos Avançados, v.10, p.227 - 236.

Mustafaraj, E.; Finn, S.; Whitlock, C.; Metaxas, P. T. (2012). Vocal minority versus silent majority: Discovering the opionions of the long tail. Privacy, Security, Risk and Trust, 2011 IEEE Third International Conference on and 2011 IEEE Third International Conference on Social Computing, Boston, EUA.

Newton, I. (1687). Princípios matemáticos da filosofia natural. Edmund Halley, Londres, Reino Unido.

O’Callaghan, L.; Mishra, N.; Meyerson, A.; Guha, S.; Motwani, R. (2002). Streaming-data algorithms for high-quality clustering. Proceedings of the 18th International Conference on Data Engineering, p. 685-694, San Jose, EUA. IEEE.

Ornstein, D. S. (1989). Ergodic theory, randomness, and "chaos". Science, v.243, n.4888, p.182-187.

Pavlidis, N.; Tasoulis, D.; Adams, N.; Hand, D. (2011). [lambda]-Perceptron: An adaptive classifier for data streams. Pattern Recognition, v.44, n.44, p.78-96. 
Pincus, S. (1991). Approximate entropy as a measure of system complexity. Proceedings of the National Academy of Sciences of the United States of America, v. 88, p. 2297-2301, Washington, EUA.

Pitman, J. (1997). Some probabilistic aspects of set partitions. American Mathematical Monthly, v.104, n.3, p.201-209.

Priebe, C.; Conroy, J.; Marchette, D.; Park, Y. (2005). Scan statistics on Enron graphs. Computational \& Mathematical Organization Theory, v.11, n.3, p.229-247.

R Development Core Team (2011). R: A Language and Environment for Statistical Computing. $\mathrm{R}$ Foundation for Statistical Computing, Vienna, Austria.

Reilly, D. L.; Cooper, L. N.; Erlbaum, C. (1982). A neural model for category learning. Biological Cybernetics, v.45, p.35-41.

Roberts, S. J. (1999). Novelty detection using extreme value statistics. IEE Proceedings on Vision, Image and Signal Processing, v.146, n.3, p.124-129.

Roberts, S. W. (1966). A comparison of some control chart procedures. Technometrics, v.8, n.3, p.411-430.

Rodrigues, P. P.; Gama, J.; Pedroso, J. (2008). Hierarchical clustering of time-series data streams. v. 20, p. 615-627, Piscataway, EUA. IEEE Educational Activities Department.

Rosen, B. E.; Soriano, D.; Bylander, T.; ; Ortiz-Zuazaga, H. (1996). Training a neural network to recognize artifacts and decelerations in cardiotocograms. Spring Symposium on Artificial Intelligence in Medicine: Applications of Current Technologies, Palo Alto, EUA.

Rousseeuw, P. J. (1987). Silhouettes: A graphical aid to the interpretation and validation of cluster analysis. Journal of Computational and Applied Mathematics, v.20, p.53 - 65.

Royden, H. L. (1968). Real Analysis. Macmillan, Nova Iorque, EUA, 2 edição.

Ryabko, D. (2010). Clustering processes. Proceedings of the 27th International Conference on Machine Learning, p. 919-926, Haifa, Israel.

Sant'anna Neto, J. L. (2003). A gênese da climatologia no Brasil: o despertar de uma ciência. Geografia, v.28, n.1.

Seneta, E. (2006). Non-negative Matrices and Markov Chains. Springer Series in Statistics. Springer, 2 edição.

Shannon, C. (1948). A mathematical theory of communication. Bell System Technical Journal, v.27, p.379-423 and 623-656. 
Shefler, W. (1988). Statistics: Concepts and Applications. The Benjamin/Cummings.

Sheikholeslami, G.; Chatterjee, S.; Zhang, A. (1998). Wavecluster: A multi-resolution clustering approach for very large spatial databases. Proceedings of the International Conference on Very Large Data Bases, p. 428-439, Nova Iorque, EUA. Citeseer.

Singh, S.; Markou, M. (2005). A black hole novelty detector for video analysis. Pattern Analysis and Applications, v.8, n.1, p.102-114.

Skvarcius, R.; Robinson, W. B. (1986). Discrete Mathematics With Computer Science Applications. Benjamin-Cummings Pub Co, Menlo Park, EUA.

Sousa, E. P. M.; Traina, A. J. M. (2006). Measuring evolving data streams behavior through their intrinsic dimension. New Generation, v.25, n.1, p.33-60.

Spinosa, E.; de Carvalho, A. (2005). Combining one-class classifiers for robust novelty detection in gene expression data. Lecture Notes in Computer Science, v.3594, p.54-64.

Spinosa, E.; de Carvalho, A.; Gama, J. (2007). OLINDDA: a cluster-based approach for detecting novelty and concept drift in data streams. Proceedings of the 2007 ACM Symposium on Applied Computing, p. 448-452, Nova Iorque, EUA. ACM.

Stanley, J. C. (1976). Computer simulation of a model of habituation. Nature, v.261, p.146-148.

Steinhaus, H. (1956). Sur la division des corp materiels en parties. Bulletin of the Polish Academy of Sciences, v.1, p.801-804.

Sun, Y.; Lu, Y. (2006). A grid-based subspace clustering algorithm for high-dimensional data streams. Web Information Systems, v. 4256 de Lecture Notes in Computer Science, p. 37-48. Springer. 10.1007/11906070_4.

Swenson Jr, G.; Kellermann, K. (1975). An Intercontinental Array-A Next-Generation Radio Telescope. Science, v.188, n.4195, p.1263.

Tarassenko, L.; Nairac, A.; Townsend, N.; Cowley, P. (1999). Novelty detection in jet engines. IEE Colloquium on Condition Monitoring: Machinery, External Structures and Health, n. 34, p. 4/1-4/5, Birmingham, Reino Unido.

Tax, D.; Duin, R. (1998). Outlier detection using classifier instability. Advances in Pattern Recognition, Lecture notes in Computer Science, v. 1451, p. 593-601, Berlim, Alemanha. Springer.

Thompson, S. (1987). Sample size for estimating multinomial proportions. The American Statistician, v.41, n.1, p.42-46. 
Tormene, P.; Giorgino, T.; Quaglini, S.; Stefanelli, M. (2009). Matching incomplete time series with dynamic time warping: an algorithm and an application to post-stroke rehabilitation. Artificial Intelligence in Medicine, v.45, n.1, p.11 - 34.

Tribus, M. (1961). Thermostatistics and Thermodynamics. D. van Nostrand Company, Inc., Princeton, EUA.

Tyson, T.; Pike, R.; Stein, M.; Szalay, A. (2002). Managing and Mining the LSST data sets. Relatório técnico, The LSST Collaboration, Tucson, EUA.

van der Pol, B. (1927). Forced oscillations in a circuit with non-linear resistance (reception with reactive triode). The London, Edinburgh, and Dublin Philosophical Magazine and Journal of Science, v.7, p.65-8.

Vapnik, V. N. (1995). The nature of statistical learning theory. Springer, Nova Iorque, EUA.

Varsta, M.; Millán, J. D. R.; Heikkonen, J. (1997). A recurrent self-organizing map for temporal sequence processing. Proceedings of the 7th International Conference on Artificial Neural Networks, p. 421-426, Londres, Reino Unido. Springer.

von Luxburg, U.; Schoelkopf, B. (2008). Statistical learning theory: Models, concepts, and results. arXiv:0810.4752v1.

Wang, W.; Yang, J.; Muntz, R. (1997). STING: A statistical information grid approach to spatial data mining. Proceedings of the International Conference on Very Large Data Bases, p. 186-195, Atenas, Grécia. Citeseer.

Ward, Joe H., J. (1963). Hierarchical grouping to optimize an objective function. Journal of the American Statistical Association, v.58, n.301, p.236-244.

Weigend, A. S.; Gershenfeld, N. S. (1993). Time Series Prediction: Forecasting the Future and Understanding the Past. Santa Fe Institute Studies in the Sciences of Complexity, Santa $\mathrm{Fe}$, EUA.

Werbos, P. J. (1993). The roots of backpropagation. Wiley-Interscience, Nova Iorque, EUA.

Wilcoxon, F. (1945). Individual comparisons by ranking methods. Biometrics Bulletin, v.1, n.6, p.80-83.

Willsky, A. (1976). A survey of design methods for failure detection in dynamic systems. Automatica, v.12, p.601-611.

Winters, P. R. (1960). Forecasting Sales by Exponentially Weighted Moving Averages. Management Science, v.6, n.3, p.324-342. 
Xu, R.; Wunsch, D. (2005). Survey of clustering algorithms. IEEE Transactions on neural networks, v.16, n.3, p.645-678.

Young, S.; Arel, I.; Karnowski, T.; Rose, D. (2010). A Fast and Stable Incremental Clustering Algorithm. 2010 Seventh International Conference on Information Technology, p. 204-209, Shanghai, China. IEEE.

Ypma, A.; Duin, R. P. W. (1997). Novelty detection using self-organizing maps. Progress in Connectionist-Based Information Systems, v. 2, p. 1322-1325. Springer, Londres.

Yu, F.; Oyana, D.; Hou, W.; Wainer, M. (2010). Approximate Clustering on Data Streams Using Discrete Cosine Transform. Journal of Information Processing Systems, v.6, p.67-78.

Zadeh, R. (2010). Towards a Principled Theory of Clustering. Unpublished. Available at: http://www.cs.cmu.edu/ rezab/papers/principled.pdf.

Zhang, T.; Ramakrishnan, R.; Livny, M. (1996). BIRCH: an efficient data clustering method for very large databases. v. 25, p. 103-114, Montreal, Canadá. ACM. 


\section{Apêndice A}

\section{A.1 Algoritmo da arquitetura Self-Organizing Novelty De- tector (SONDE)}

O Algoritmo 5 apresenta a implementação da arquitetura SONDE adotada nos experimentos desta tese. Esse algoritmo avalia cada vetor de entrada $\vec{I}(t)$ em busca pelo neurônio Best-Matching Unit, i.e, $c_{\mathrm{Bmu}}$. Esse neurônio adapta-se segundo as Equações 4.2, 4.1] e 4.4. Caso nenhum dos neurônios seja capaz de representar o padrão de entrada $\vec{I}(t)$, um novo neurônio é criado. No cálculo de distância entre padrões de entrada e protótipos de neurônios, utiliza-se a norma euclidiana.

A ordem de complexidade da SONDE, obtida a partir do Algoritmo 5, é similar à complexidade de família de algoritmos Winner Takes All, entre eles, Adaptive Resonance Theory (Carpenter et al., 1991) e Grow When Required (Marsland, 2002). Nesses algoritmos existe a necessidade de competição entre neurônios para definir qual se torna o Best-Matching Unit. Devido a esse fato, a complexidade do algoritmo depende quadraticamente do número de neurônios, ou seja, $k=|K|$, que, por sua vez depende do número $t$ de padrões de entrada. Dessa forma, a ordem de complexidade de tempo da arquitetura SONDE é $O\left(k^{2}(t)\right)$ e a ordem de complexidade de espaço é $O(k(t))$. 


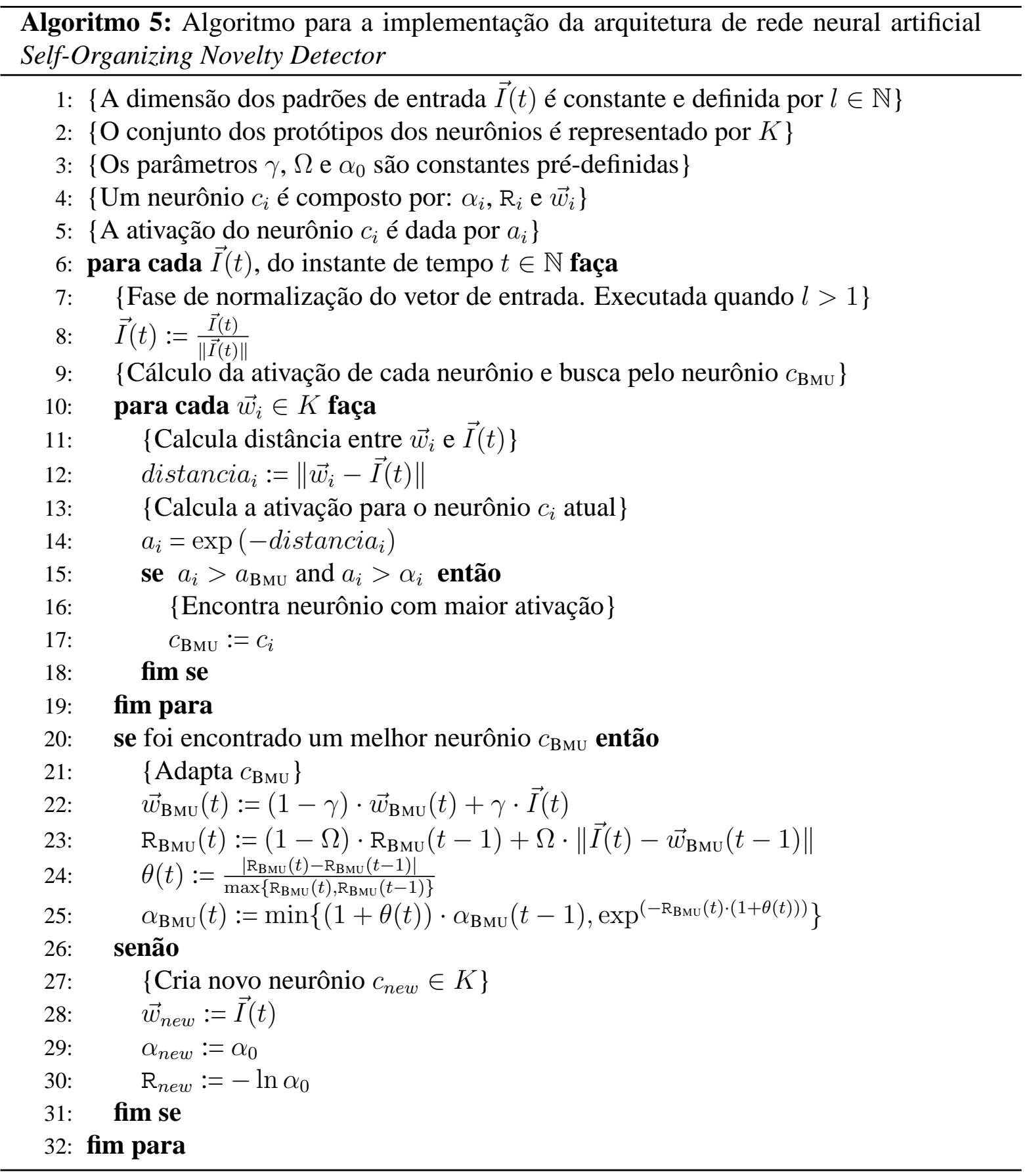


A

abordagem, 5, 40, 88

adaptação de parâmetros de aprendizado, 47 adaptação de partições, 46

adaptação do viés indutivo, 53, 62

adaptação incremental, 49, 58

Adaptive Resonance Theory, 4

agrupamento, 2

agrupamento de dados, 9

agrupamento de fluxo de dados, 3

agrupamento de fluxos de dados, 15

agrupamento de processos estocásticos, 15

agrupamento hierárquico, 10

agrupamento incremental, 24, 47

agrupamento partitivo, 10

agrupamentos nebulosos, 2, 13

alcançar, 17

algoritmo de agrupamento, 11

análise, 1

anomalia, 9, 30

Aprendizado de Máquina, 30

Árvore B, 21

atributos, 2, 10

avaliação de agrupamentos, 17

B

Best-Matching Unit, 23

BIRCH, 21, 67 blocos de subsequências, 6, 42

\section{C}

cadeias de Markov, 6, 41, 50, 63

camada competitiva, 48

camada de armazenamento, 48

características, 10

Clustream, 21, 67

coerência, 18, 67

coleta de dados, 11, 46

Completude, 13, 14, 19, 26

complexidade de tempo e espaço, 10

comportamento, 4, 15

comportamento assintótico, 41

concept drift, 4

Concept Feature Tree, 21

conjunto de elementos, 2

conjunto universo, 2

conjuntos finitos, 15

conjuntos tradicionais de dados, 3, 12

Consistência, 13, 14

continuidade, 17, 26

convergência de aprendizado, 54

D

Data Stream Dynamic Clustering, 7, 41, 63

dendrograma, 10,45

desvio abrupto, 30 
detecção de intrusão, 12

detecção de novidades, 30, 52

dimensões, 10

dispersão de padrões, 21

dispersão média dos padrões de entrada, 48

dissimilaridades, 14

distância euclidiana, 10, 45, 78

DSDC, 41, 69

DTw, 78

Dynamic Time Warping, 78

E

elemento, 2

entropia de Shannon, 6

espaço de representação da linguagem, 44

especialista, 2, 4, 5, 81

especialização, 49, 56

esquecimento, 74

estabilidade, 84

estabilidade de dados, 15

estados comportamentais, 47, 50

estados de comportamento, 63

estruturas de conhecimento, 39, 48, 54

exceção, 30

F

falha, 30

fator de esquecimento, 75

features, 10

fenômeno, 1, 9

fenômenos complexos, 27

fenômenos estáveis, 29

fluxos de dados, 3, 9, 18, 68

formalização, 9, 15

função de dissimilaridade, 15

função de distância, 11

\section{G}

generalização, 50

gradiente descendente, 41, 53
Grow When Required, 4, 53

grupos, 10, 39

Gwr, 69

\section{H}

hierarquia de agrupamentos, 10

hipótese, 2, 5, 40, 88

I

incremental, 6

indução, 1

indução contínua, 3

indutor, 2

informações inesperadas, 30

Invariância à Escala, 13

Invariância Espaço-temporal, 26

isomorfismo, 42

J

janelas deslizantes, 70

$\mathbf{K}$

K-médias, 6, 11, 21, 70,

$\mathbf{L}$

limiar de ativação, 48, 49

linguagem de representação de hipóteses, 2,

44

M

matrix de dissimilaridades, 13

matriz de distâncias, 13

matriz de probabilidades de transições, 51

média de padrões, 21

média móvel exponencial ponderada, 49, 75

medida, 69

medida de avaliação de grupos, 10

medida de avaliação de qualidade, 18

medida de variações comportamentais, 5

medidas de avaliação de agrupamento, 69

medidas de novidades temporais, 36

memória, 74 
métodos não supervisionados, 1

métodos supervisionados, 1

microagrupamento, 21

microblogging, 12

microgrupos, 21

MMEP, 75, 78

modelo, 1, 10, 44

modelo de conhecimento, 30

modelo ideal, 44, 45, 78, 82

$\mathbf{N}$

natureza sequencial, 15

Navalha de Occam, 44

neurônio da SONDE, 41

norma euclidiana, 48

novidades não temporais, 32

novidades temporais, 32, 53

O

OLINDDA, 22

OPTIGRID, 21

outlier, 30

$\mathbf{P}$

padrão de entrada, 47, 75

parâmetro de ponderação, 75, 80

parâmetros subótimos, 81

partição, 2, 13, 74

partições consecutivas, 17

partições contínuas, 18

premissas, 2

pré-processamento, 47

probabilidade de transição, 63

probabilidades assintóticas, 57

processo de coleta dos dados, 3

propriedade, 12

Propriedade da Consistência, 74

propriedade de agrupamento, 26

propriedade de convergência, 57

protótipo, 11, 17, 39, 49
$\mathbf{R}$

raio médio, 48, 49

refinamento, 17

regra de adaptação, 60

ruído, 30

\section{$\mathbf{S}$}

Self-Organizing Maps, 53

Self-Organizing Novelty Detector, 6, 40, 88

separabilidade, 67

sequência contínua de partições, 18

sequência de partições, 15

Silhueta, 7, 41, 42, 67, 69

sobrejeção, 14

SONDE, 40, 54, 88

STING, 21

surpresa, 33

$\mathbf{T}$

taxa de aprendizado, 23, 37

tendência, 49

teorema de impossibilidade, 14

Teorema de Perron-Frobenius, 64

Teoria dos Conjuntos, 9, 12, 25

teste de hipótese, 70

V

variação de comportamento, 58, 63, 71, 84

variação de entropia, 41, 52, 57

variações comportamentais, 30

variações comportamentais de fluxos de da$\operatorname{dos}, 38$

variações de comportamento, 4, 5, 18

vetor-gradiente, 54, 58, 60

viés de código, 44

viés de parâmetros, 44, 46, 60

viés de preferência, 44

viés de preferência de código, 44

viés indutivo, 3, 4, 38

viés indutivo de linguagem, 44 
W

WAVECluster, 21

Wavelet, 21

WCSS, 69

Winner Takes All,4

Within-Cluster Sum of Squares, 69 